\title{
A new second-order upper bound for the ground state energy of dilute Bose gases
}

\author{
Giulia Basti $^{(\mathbb{D})}{ }$, Serena Cenatiempo ${ }^{(\mathbb{D})}$ and Benjamin Schlein ${ }^{\left(D_{3}\right.}$ \\ ${ }^{1}$ Mathematics Area, Gran Sasso Science Institute, Viale Francesco Crispi 7, 67100 L’Aquila, Italy; E-mail: giulia.basti@gssi.it. \\ 2 Mathematics Area, Gran Sasso Science Institute, Viale Francesco Crispi 7, 67100 L'Aquila, Italy; \\ E-mail: serena.cenatiempo@gssi.it. \\ ${ }^{3}$ Institute of Mathematics, University of Zurich, Winterthurerstrasse 190, 8057 Zurich, Switzerland; \\ E-mail: benjamin.schlein@math.uzh.ch.
}

Received: 3 March 2021; Revised: 31 August 2021; Accepted: 14 September 2021

Keywords: dilute Bose gases, Lee-Huang-Yang formula, Thermodynamic limit, Interacting bosons, Bose-Einstein condensation 2020 Mathematics subject classification: 46N50, 81V70, 81V73, 82B10, 82B27, 82D03

\begin{abstract}
We establish an upper bound for the ground state energy per unit volume of a dilute Bose gas in the thermodynamic limit, capturing the correct second-order term, as predicted by the Lee-Huang-Yang formula. This result was first established in [20] by H.-T. Yau and J. Yin. Our proof, which applies to repulsive and compactly supported $V \in L^{3}\left(\mathbb{R}^{3}\right)$, gives better rates and, in our opinion, is substantially simpler.
\end{abstract}

\section{Introduction and main result}

We consider $N$ bosons in a finite box $\Lambda_{L}=\left[-\frac{L}{2}, \frac{L}{2}\right]^{3} \subset \mathbb{R}^{3}$, interacting via a two-body nonnegative, radial, compactly supported potential $V$ with scattering length $\mathfrak{a}$. The Hamilton operator has the form

$$
H_{L}=-\sum_{i=1}^{N} \Delta_{i}+\sum_{1 \leq i<j \leq N} V\left(x_{i}-x_{j}\right)
$$

and acts on the Hilbert space $L_{S}^{2}\left(\Lambda_{L}^{N}\right)$, the subspace of $L^{2}\left(\Lambda_{L}^{N}\right)$ consisting of functions that are symmetric with respect to permutations of the $N$ particles (we use here units with particle mass $m=1 / 2$ and $\hbar=1)$. We assume Dirichlet boundary conditions and denote by $E(N, L)$ the corresponding ground state energy. We are interested in the energy per unit volume in the thermodynamic limit, defined by

$$
e(\rho)=\lim _{\substack{N, L \rightarrow+\infty \\ \rho=N / L^{3}}} \frac{E(N, L)}{L^{3}} .
$$

Bogoliubov [4] and, later, in more explicit terms, Lee-Huang-Yang [14] predicted that, in the dilute limit $\rho \mathfrak{a}^{3} \ll 1$, the specific ground state energy (1.2) is so that

$$
e(\rho)=4 \pi \mathfrak{a} \rho^{2}\left[1+\frac{128}{15 \sqrt{\pi}}\left(\rho \mathfrak{a}^{3}\right)^{1 / 2}+o\left(\left(\rho \mathfrak{a}^{3}\right)^{1 / 2}\right)\right] .
$$

\footnotetext{
(C) The Author(s), 2021. Published by Cambridge University Press. This is an Open Access article, distributed under the terms of the Creative Commons Attribution licence (https://creativecommons.org/licenses/by/4.0/), which permits unrestricted re-use, distribution, and reproduction in any medium, provided the original work is properly cited.
} 
In particular, up to lower order corrections, it only depends on the interaction potential through the scattering length $\mathfrak{a}$. An alternative heuristic derivation of (1.3) was proposed in [15] (this approach was based on a partial differential equation with a convolution nonlinearity, whose mathematical properties were recently studied in [7]).

On the rigorous level, the validity of the leading term on the right-hand side of (1.3) was established by Dyson, who obtained an upper bound in [8], and by Lieb-Yngvason, who proved the matching lower bound in [16]. An upper bound with the correct second-order contribution was first derived in [20] by Yau-Yin for regular potentials, improving a previous estimate from [9], which only recovered the correct formula (as an upper bound) in the limit of weak coupling. The approach of [20] has been reviewed and adapted to a grand canonical setting in [1]. As for the lower bound, preliminary results have been obtained in [13] and [6], where (1.3) was shown in particular regimes, where the potential scales with the density $\rho$. Finally, a rigorous lower bound matching (1.3) has been obtained by Fournais-Solovej, in [10] for $L^{1}$ potentials and, very recently, in [11] for a hard sphere interaction (a nonoptimal bound for hard spheres had been previously obtained in [5]).

Our goal in this article is to show a new upper bound for (1.3). With respect to the upper bound established in [20], our result holds for a larger class of potentials (in [20], the upper bound is proven for smooth potentials), it gives a better rate (although still far from optimal) and, most important in our opinion, it relies on a simpler proof.

Theorem 1.1. Let $V \in L^{3}\left(\mathbb{R}^{3}\right)$ be nonnegative, radially symmetric, with $\operatorname{supp}(V) \subset B_{R}(0)$ and scattering length $\mathfrak{a}$. Then, the specific ground state energy $e(\rho)$ of the Hamilton operator $H_{L}$ defined in (1.1) satisfies

$$
e(\rho) \leq 4 \pi \rho^{2} \mathfrak{a}\left[1+\frac{128}{15 \sqrt{\pi}}\left(\rho \mathfrak{a}^{3}\right)^{1 / 2}\right]+C \rho^{5 / 2+1 / 10}
$$

for some $C>0$ (depending on $\|V\|_{3}$ and on $R$ ) and for $\rho$ small enough.

Remark. Since Dirichlet boundary conditions lead to the largest energy, the upper bound (1.4) holds in fact for arbitrary boundary conditions.

Remark. At the cost of a longer proof, we could improve the bound on the error, up to the order $\rho^{5 / 2+2 / 9}$ (this is the rate determined by Lemma 5.1).

The proof of 1.4 is based on the construction of an appropriate trial state. However, we do not directly construct a trial state in $L_{s}^{2}\left(\Lambda_{L}^{N}\right)$ for the Hamiltonian (1.1). Instead, to simplify the analysis, it is very convenient to (1) consider smaller boxes (rather than letting $N, L \rightarrow \infty$ first and considering small $\rho$ at the end, we will consider a diagonal limit, with $L=\rho^{-\gamma}$, for some $\gamma>1$ ), (2) work with periodic rather than Dirichlet boundary conditions and (3) work in the grand-canonical setting, considering states with variable number of particles, rather than the canonical setting. In other words, our trial state will be defined on the bosonic Fock space

$$
\mathcal{F}\left(\Lambda_{L}\right)=\bigoplus_{n \geq 0} L_{s}^{2}\left(\Lambda_{L}^{n}\right)=\bigoplus_{n \geq 0} L^{2}\left(\Lambda_{L}\right)^{\otimes_{S} n}
$$

where $L_{s}^{2}\left(\Lambda_{L}^{n}\right)$ is the subspace of $L^{2}\left(\Lambda_{L}^{n}\right)$ consisting of wave functions that are symmetric with respect to permutations. On $\mathcal{F}\left(\Lambda_{L}\right)$, we consider the number of particles operator $\mathcal{N}$ defined through $(\mathcal{N} \psi)^{(n)}=$ $n \psi^{(n)}$. Moreover, we introduce the Hamiltonian operator $\mathcal{H}$, setting

$$
(\mathcal{H} \psi)^{(n)}=\mathcal{H}^{(n)} \psi^{(n)}
$$


with

$$
\mathcal{H}^{(n)}=\sum_{j=1}^{n}-\Delta_{x_{j}}+\sum_{1 \leq i<j \leq n} V\left(x_{i}-x_{j}\right),
$$

imposing now (in contrast to what we did in (1.1)) periodic boundary conditions (with a slight abuse of notation, $V$ denotes here the periodic extension of the potential introduced in (1.1)). The upper bound for the energy of (1.5) will then imply Theorem 1.1 thanks to the following localisation result.

Proposition 1.2. Let $e(\rho)$ be defined as in (1.2), with Dirichlet boundary conditions. Let $R<b<L$, with $R$ the radius of the support of the potential $V$, as defined in Theorem 1.1. Then, for any normalised $\Psi_{L} \in \mathcal{F}\left(\Lambda_{L}\right)$ satisfying periodic boundary conditions and such that

$$
\left\langle\Psi_{L}, \mathcal{N} \Psi_{L}\right\rangle \geq \rho\left(1+c^{\prime} \rho\right)(L+2 b+R)^{3}, \quad\left\langle\Psi_{L}, \mathcal{N}^{2} \Psi_{L}\right\rangle \leq C^{\prime} \rho^{2}(L+2 b+R)^{6}
$$

for some $c^{\prime}, C^{\prime}>0$, we have

$$
e(\rho) \leq \frac{\left\langle\Psi_{L}, \mathcal{H} \Psi_{L}\right\rangle}{L^{3}}+\frac{C}{L^{4} b}\left\langle\Psi_{L}, \mathcal{N} \Psi_{L}\right\rangle
$$

for a universal constant $C>0$.

The proof of Proposition 1.2 is standard; see [18, 20,1]. Roughly speaking, the idea consists in using $\Psi_{L}$ (satisfying periodic boundary conditions on the box $\Lambda_{L}$ ) to construct a trial state satisfying Dirichlet boundary conditions on a slightly larger box of side length $L+2 b$ and then in approaching the thermodynamic limit by replicating the Dirichlet state on several boxes of side length $L+2 b$, separated by corridors of size $R$ (to avoid interactions among different boxes). For completeness, we provide a detailed proof of Proposition 1.2 in Appendix A.

The bulk of the article contains the proof of the following proposition, establishing the existence of a trial state with the correct energy per unit volume and the correct expected number of particles on boxes of size $L=\tilde{\rho}^{-\gamma}$. We use here the notation $\tilde{\rho}$ for the density to stress the fact that the upper bound (1.9) will be inserted in (1.7) to prove an upper bound for the specific ground state energy $e(\rho)$, for a slightly different density $\rho<\tilde{\rho}$ (to make up for the corrections on the right-hand side of (1.6)).

Proposition 1.3. As in Theorem 1.1, assume that $V \in L^{3}\left(\mathbb{R}^{3}\right)$ is nonnegative, radially symmetric with supp $V \subset B_{R}(0)$ and scattering length a. For $\gamma>1$ and $\tilde{\rho}>0$ let $L=\tilde{\rho}^{-\gamma}$. Then, for every $0<\varepsilon<1 / 4$, there exists $\Phi_{\tilde{\rho}} \in \mathcal{F}\left(\Lambda_{L}\right)$ satisfying periodic boundary conditions such that

$$
\left\langle\Phi_{\tilde{\rho}}, \mathcal{N} \Phi_{\tilde{\rho}}\right\rangle \geq \tilde{\rho} L^{3}, \quad\left\langle\Phi_{\tilde{\rho}}, \mathcal{N}^{2} \Phi_{\tilde{\rho}}\right\rangle \leq C \tilde{\rho}^{2} L^{6}
$$

and

$$
\frac{\left\langle\Phi_{\tilde{\rho}}, \mathcal{H} \Phi_{\tilde{\rho}}\right\rangle}{L^{3}} \leq 4 \pi \mathfrak{a} \tilde{\rho}^{2}\left(1+\frac{128}{15 \sqrt{\pi}}\left(\mathfrak{a}^{3} \tilde{\rho}\right)^{1 / 2}\right)+\mathcal{E},
$$

with

$$
\mathcal{E} \leq C \tilde{\rho}^{5 / 2} \cdot \max \left\{\tilde{\rho}^{\varepsilon}, \tilde{\rho}^{4-3 \gamma-6 \varepsilon}, \tilde{\rho}^{9 / 4-3 \gamma / 2-3 \varepsilon}\right\}
$$

Remark. the condition $\gamma>1$ is needed to make sure that the localisation error in (1.7) is negligible. While we will choose $\gamma=11 / 10$ to optimise the rate, our analysis allows us to take any $1<\gamma<4 / 3$. With a longer proof, our techniques could be extended to all $1<\gamma<5 / 3$. This suggests that our trial state captures the correct correlations of the ground state, up to length scales of the order $\rho^{-5 / 3}$.

With Proposition 1.2 and Proposition 1.3 we can prove Theorem 1.1. 
Proof of Theorem 1.1. For given $\rho>0$, we would like to choose $\tilde{\rho}$ or, equivalently, $L=\tilde{\rho}^{-\gamma}$, so that (1.8) implies (1.6). Fixing $c^{\prime}>0$ and $b=L^{\alpha}$, for some $\alpha \in(0 ; 1)$, this leads to the implicit equation

$$
L=\tilde{\rho}^{-\gamma}=\left[\rho\left(1+c^{\prime} \rho\right)\left(1+2 L^{\alpha-1}+R L^{-1}\right)^{3}\right]^{-\gamma} .
$$

Setting $L=\left(\rho\left(1+c^{\prime} \rho\right)\right)^{-\gamma} x$, we rewrite (1.10) as

$$
x=\left(1+2\left(\rho\left(1+c^{\prime} \rho\right)\right)^{\gamma(1-\alpha)} / x^{1-\alpha}+R\left(\rho\left(1+c^{\prime} \rho\right)\right)^{\gamma} / x\right)^{-3 \gamma}
$$

and we conclude that the existence of a solution $L=L(\rho)$ of (1.10) follows from the implicit function theorem, if $\rho>0$ is small enough (the solution stems from $x=1$ for $\rho=0$ ). By construction, $L=\tilde{\rho}^{-\gamma}$, with

$$
\tilde{\rho}=\rho\left(1+c^{\prime} \rho\right)\left(1+2 \tilde{\rho}^{\gamma(1-\alpha)}+R \tilde{\rho}^{\gamma}\right)^{3}
$$

and thus

$$
\rho \leq \tilde{\rho} \leq \rho\left(1+C \rho+C \rho^{\gamma(1-\alpha)}\right) .
$$

From Proposition 1.3, we find $\Phi_{\tilde{\rho}} \in \mathcal{F}\left(\Lambda_{L}\right)$ such that (1.8) and (1.9) hold true. In particular, (1.8) implies (1.6) (with $b=L^{\alpha}, C^{\prime}=C$ ). Thus, from Proposition 1.2 we conclude

$$
e(\rho) \leq \frac{\left\langle\Phi_{\tilde{\rho}}, \mathcal{H} \Phi_{\tilde{\rho}}\right\rangle}{L^{3}}+\frac{C}{L^{4} b}\left\langle\Phi_{\tilde{\rho}}, \mathcal{N} \Phi_{\tilde{\rho}}\right\rangle
$$

Inserting (1.9) and (1.8), we obtain (since (1.8) also implies that $\left\langle\Phi_{\tilde{\rho}}, \mathcal{N} \Phi_{\tilde{\rho}}\right\rangle \leq C \widetilde{\rho} L^{3}$ )

$$
e(\rho) \leq 4 \pi \mathfrak{a} \tilde{\rho}^{2}\left[1+\frac{128}{15 \sqrt{\pi}}\left(\mathfrak{a}^{3} \tilde{\rho}\right)^{1 / 2}\right]+C \tilde{\rho}^{1+\gamma(1+\alpha)}+C \tilde{\rho}^{5 / 2} \cdot \max \left\{\tilde{\rho}^{\varepsilon}, \tilde{\rho}^{4-3 \gamma-6 \varepsilon}, \tilde{\rho}^{9 / 4-3 \gamma / 2-3 \varepsilon}\right\} .
$$

With (1.11), we conclude that

$$
\begin{aligned}
e(\rho) \leq & 4 \pi \mathfrak{a} \rho^{2}\left[1+\frac{128}{15 \sqrt{\pi}}\left(\mathfrak{a}^{3} \rho\right)^{1 / 2}\right] \\
& +C \rho^{5 / 2} \cdot \max \left\{\rho^{\gamma(1-\alpha)-1 / 2}, \rho^{\gamma(1+\alpha)-3 / 2}, \rho^{\varepsilon}, \rho^{4-3 \gamma-6 \varepsilon}, \rho^{9 / 4-3 \gamma / 2-3 \varepsilon}\right\},
\end{aligned}
$$

where we neglected errors of order $C \rho^{3}$, which are subleading compared with $C \rho^{5 / 2+\varepsilon}$, since $\varepsilon \in(0 ; 1 / 4)$.

Comparing the first two errors, we choose $\alpha=1 /(2 \gamma)$. Comparing instead third and fourth errors, we set $\varepsilon=(4-3 \gamma) / 7$ (both choices are consistent with the conditions $\alpha \in(0 ; 1)$ and $\varepsilon \in(0 ; 1 / 4)$, because $\gamma>1)$. Since, with these choices, the last error is of smaller order, we obtain

$$
e(\rho) \leq 4 \pi \mathfrak{a} \rho^{2}\left[1+\frac{128}{15 \sqrt{\pi}}\left(\mathfrak{a}^{3} \rho\right)^{1 / 2}\right]+C \rho^{5 / 2} \cdot \max \left\{\rho^{\gamma-1}, \rho^{(4-3 \gamma) / 7}\right\} .
$$

Choosing $\gamma=11 / 10$, we find (1.4).

The proof of Proposition 1.3 occupies the rest of the article (excluding Appendix A, where we show Proposition 1.2). In Section 2 we define our trial state. To this end, we will start with a coherent state describing the Bose-Einstein condensate. Similar to [12,9], we will then act on the coherent state with a Bogoliubov transformation to add the expected correlation structure. Finally, we will apply the exponential of a cubic expression in creation operators. While the Bogoliubov transformation creates pairs of excitations with opposite momenta $p,-p$, the cubic operator creates three excitations at a time, two with large momenta $r+v,-r$ and one with low momentum $v$. This last step is essential, since, as 
follows from [9, 17], quasi-free states cannot approximate the ground state energy to the precision of (1.3). We remark that the idea of creating triples of excitations originally appeared in the work of YauYin [20] (a brief comparison with the trial state of [20] can be found after the precise definition of our trial state in (2.25)). Recently, it has been also applied to establish the validity of Bogoliubov theory in the Gross-Pitaevskii regime in [3, 2]; while our approach is inspired by these papers, we need new tools to deal with the large boxes considered in Proposition 1.3 (a simple computation shows that the GrossPitaevskii regime corresponds to the exponent $\gamma=1 / 2$; to control localisation errors, we need instead to choose $\gamma>1$ ). In Section 3, we combine the contributions to the energy of the trial state arising from the conjugation with the Bogoliubov transformation and from the action of the cubic phase, proving the desired upper bound. In Section 4 and Section 5, we prove technical bounds which allow us to identify the leading contributions collected in Section 3.

\section{Setting and trial state}

To show Proposition 1.3, we find it convenient to work with rescaled variables. We consider the transformation $x_{j} \rightarrow x_{j} / L$ and, motivated by the choice $L=\tilde{\rho}^{-\gamma}$ in Proposition 1.3, we set $N=\tilde{\rho}^{1-3 \gamma}$ (we will look for trial states with expected number of particles close to $N$ to make sure that (1.8) holds true). It follows that the Hamiltonian (1.5) is unitarily equivalent to the operator $L^{-2} \mathcal{H}_{N}=\tilde{\rho}^{2 \gamma} \mathcal{H}_{N}$, with $\mathcal{H}_{N}$ acting on the Fock space $\mathcal{F}(\Lambda)$ defined over the unit box $\Lambda=\Lambda_{1}=[-1 / 2 ; 1 / 2]^{3}$ (with periodic boundary conditions) so that $\left(\mathcal{H}_{N} \Psi\right)^{(n)}=\mathcal{H}_{N}^{(n)} \Psi^{(n)}$, with

$$
\mathcal{H}_{N}^{(n)}=\sum_{j=1}^{n}-\Delta_{x_{j}}+\sum_{1 \leq i, j \leq n} N^{2-2 \kappa} V\left(N^{1-\kappa}\left(x_{i}-x_{j}\right)\right)
$$

and $\kappa=(2 \gamma-1) /(3 \gamma-1)$. The assumption $\gamma>1$ in Proposition 1.3 allows us to restrict our attention to $k \in(1 / 2 ; 2 / 3)$.

For any momentum $p \in \Lambda^{*}=2 \pi \mathbb{Z}^{3}$, we introduce on the Fock space $\mathcal{F}(\Lambda)=\bigoplus_{n \geq 0} L_{s}^{2}\left(\Lambda^{n}\right)$ the operators $a_{p}^{*}, a_{p}$, creating and, respectively, annihilating a particle with momentum $p$. Creation and annihilation operators satisfy the canonical commutation relations

$$
\left[a_{p}, a_{q}^{*}\right]=\delta_{p q}, \quad\left[a_{p}, a_{q}\right]=\left[a_{p}^{*}, a_{q}^{*}\right]=0 .
$$

On $\mathcal{F}(\Lambda)$, we define the number of particles operator $\mathcal{N}=\sum_{p \in \Lambda^{*}} a_{p}^{*} a_{p}$. Expressed in terms of creation and annihilation operators, the Hamiltonian $\mathcal{H}_{N}$ takes the form

$$
\mathcal{H}_{N}=\sum_{p \in \Lambda^{*}} p^{2} a_{p}^{*} a_{p}+\frac{1}{2 N^{1-\kappa}} \sum_{p, q, r \in \Lambda^{*}} \widehat{V}\left(r / N^{1-\kappa}\right) a_{p+r}^{*} a_{q}^{*} a_{q+r} a_{p} .
$$

We now construct our trial state. To generate a condensate, we use a Weyl operator

$$
W_{N_{0}}=\exp \left[\sqrt{N_{0}} a_{0}^{*}-\sqrt{N_{0}} a_{0}\right]
$$

with a parameter $N_{0}$ to be specified later on. While $W_{N_{0}}$ leaves $a_{p}, a_{p}^{*}$ invariant, for all $p \in \Lambda^{*} \backslash\{0\}$, it produces shifts of $a_{0}, a_{0}^{*}$; in other words,

$$
W_{N_{0}}^{*} a_{0} W_{N_{0}}=a_{0}+\sqrt{N_{0}}, \quad W_{N_{0}}^{*} a_{0}^{*} W_{N_{0}}=a_{0}^{*}+\sqrt{N_{0}} .
$$

When acting on the vacuum vector $\Omega=\{1,0, \ldots\}$, (2.3) generates a coherent state in the zeromomentum mode $\varphi_{0}(x) \equiv 1$, with expected number of particles $N_{0}$.

It turns out, however, that the coherent state does not approximate the ground state energy, not even to leading order. To get closer to the ground state energy, it is crucial to add correlations among particles. 
To this end, we fix $0<\ell<1 / 2$ and we consider the lowest energy solution $f_{\ell}$ of the Neumann problem

$$
\left[-\Delta+\frac{1}{2} V\right] f_{\ell}=\lambda_{\ell} f_{\ell}
$$

on the ball $|x| \leq N^{1-\kappa} \ell$, with the normalisation $f_{\ell}(x)=1$ if $|x|=N^{1-\kappa} \ell$. Furthermore, by rescaling, we define $f_{N, \ell}(x):=f_{\ell}\left(N^{1-\kappa} x\right)$ for $|x| \leq \ell$. We extend $f_{N, \ell}$ to a function on $\Lambda$, by fixing $f_{N, \ell}(x)=1$, for all $x \in \Lambda$, with $|x|>\ell$. Then

$$
\left[-\Delta+\frac{1}{2} N^{2-2 \kappa} V\left(N^{1-\kappa} x\right)\right] f_{N, \ell}(x)=N^{2-2 \kappa} \lambda_{\ell} f_{N, \ell}(x) \chi_{\ell}(x)
$$

for all $x \in \Lambda$, where $\chi_{\ell}$ denotes the characteristic function of the ball of radius $\ell$. We denote by $\widehat{f}_{N, \ell}(p)$ the Fourier coefficients of the function $f_{N, \ell}$, for $p \in \Lambda^{*}$. We also define $w_{\ell}(x)=1-f_{\ell}(x)$ (with $w_{\ell}(x)=0$ for $\left.|x|>N^{1-\kappa} \ell\right)$ and its rescaled version $w_{N, \ell}: \Lambda \rightarrow \mathbb{R}$ through $w_{N, \ell}(x)=w_{\ell}\left(N^{1-\kappa} x\right)=1-f_{N, \ell}(x)$. The Fourier coefficients of $w_{N, \ell}$ are given by

$$
\widehat{w}_{N, \ell}(p)=\int_{\Lambda} w_{\ell}\left(N^{1-\kappa} x\right) e^{-i p \cdot x} d x=\frac{1}{N^{3-3 \kappa}} \widehat{w}_{\ell}\left(p / N^{1-\kappa}\right)
$$

where $\widehat{w}_{\ell}(k)$ denotes the Fourier transform of the (compactly supported) function $w_{\ell}$. Some important properties of the solution of the eigenvalue problem (2.5) are summarised in the following lemma, whose proof can be found in [3, Appendix A] (replacing $N \in \mathbb{N}$ by $N^{1-\kappa}$ ).

Lemma 2.1. Let $V \in L^{3}\left(\mathbb{R}^{3}\right)$ be nonnegative, compactly supported and spherically symmetric. Fix $\ell>0$ and let $f_{\ell}$ denote the solution of (2.5). For $N \in \mathbb{N}$ large enough, the following properties hold true:

i) We have

$$
\left|\lambda_{\ell}-\frac{3 \mathfrak{a}}{N^{3-3 \kappa} \ell^{3}}\right| \leq \frac{1}{N^{3-3 \kappa} \ell^{3}} \frac{C \mathfrak{a}^{2}}{\ell N^{1-\kappa}} .
$$

ii) We have $0 \leq f_{\ell}, w_{\ell} \leq 1$. Moreover, there exists a constant $C>0$ such that

$$
\left|\int V(x) f_{\ell}(x) d x-8 \pi \mathfrak{a}\right| \leq \frac{C \mathfrak{a}^{2}}{\ell N^{1-\kappa}} .
$$

iii) There exists a constant $C>0$ such that, for all $x \in \mathbb{R}^{3}$,

$$
w_{\ell}(x) \leq \frac{C}{|x|+1} \quad \text { and } \quad\left|\nabla w_{\ell}(x)\right| \leq \frac{C}{x^{2}+1} .
$$

iv) There exists a constant $C>0$ such that, for all $p \in \mathbb{R}^{3}$,

$$
\left|\widehat{w}_{N, \ell}(p)\right| \leq \frac{C}{N^{1-\kappa} p^{2}} .
$$

We consider the coefficients $\eta: \Lambda^{*} \rightarrow \mathbb{R}$ defined through

$$
\eta_{p}=-N \widehat{w}_{N, \ell}(p)=-\frac{N^{\kappa}}{N^{2-2 \kappa}} \widehat{w}_{\ell}\left(p / N^{1-\kappa}\right)
$$

Lemma 2.1 implies that

$$
\left|\eta_{p}\right| \leq \frac{C N^{\kappa}}{p^{2}}
$$


for all $p \in \Lambda_{+}^{*}=2 \pi \mathbb{Z}^{3} \backslash\{0\}$ and for some constant $C>0$ independent of $N \in \mathbb{N}$ (for $N \in \mathbb{N}$ large enough). From (2.6), we find the relation

$$
p^{2} \eta_{p}+\frac{N^{\kappa}}{2} \widehat{V}\left(p / N^{1-\kappa}\right)+\frac{1}{2 N} \sum_{q \in \Lambda^{*}} N^{\kappa} \widehat{V}\left((p-q) / N^{1-\kappa}\right) \eta_{q}=N^{3-2 \kappa} \lambda_{\ell}\left(\widehat{\chi}_{\ell} * \widehat{f}_{N, \ell}\right)(p) .
$$

From Lemma 2.1, part iii), we also obtain

$$
\left|\eta_{0}\right| \leq N^{3-2 \kappa} \int_{\mathbb{R}^{3}} w_{\ell}(x) d x \leq C N^{\kappa}
$$

The coefficients $\eta_{p}$ will be used to model, through a Bogoliubov transformation, short-distance correlations among particles. To reach this goal, it is enough to act on momenta $|p| \gg N^{\kappa / 2}$. On low momenta, the Bogoliubov transformation is needed to diagonalise the (renormalised) quadratic part of the Hamiltonian. For $\varepsilon>0$ small enough, we therefore define the set

$$
P_{L}=\left\{p \in \Lambda_{+}^{*}:|p| \leq N^{\kappa / 2+\varepsilon}\right\}
$$

of low momenta. We will denote its complement by $P_{L}^{c}=\Lambda_{+}^{*} \backslash P_{L}$. For $p \in \Lambda_{+}^{*}$ we set

$$
v_{p}=\tau_{p} \chi\left(p \in P_{L}\right)+\eta_{p} \chi\left(p \in P_{L}^{c}\right)
$$

with $\eta_{p}$ defined in (2.7), $\tau_{p} \in \mathbb{R}$ defined by

$$
\tanh \left(2 \tau_{p}\right)=-\frac{8 \pi \mathfrak{a} N^{\kappa}}{p^{2}+8 \pi \mathfrak{a} N^{\kappa}}
$$

and $\chi(p \in S)$ denoting the indicator function of the set $S$. With these coefficients, we define the Bogoliubov transformation

$$
T_{v}=\exp \left(\frac{1}{2} \sum_{p \in \Lambda_{+}^{*}} v_{p}\left(a_{p}^{*} a_{-p}^{*}-\text { h.c. }\right)\right)
$$

For any $p \neq 0$ we have

$$
T_{v}^{*} a_{p} T_{v}=\gamma_{p} a_{p}+\sigma_{p} a_{-p}^{*}
$$

with the notation $\gamma_{p}=\cosh \left(v_{p}\right)$ and $\sigma_{p}=\sinh \left(v_{p}\right)$.

With the Weyl operator (2.3) and the Bogoliubov transformation (2.13), we obtain the 'squeezed' coherent state $\widetilde{\Psi}_{N}=W_{N_{0}} T_{\nu} \Omega$. Choosing $N_{0}$ so that $N=N_{0}+\|\sigma\|^{2}$, one can show that this trial state has approximately $N$ particles and, to leading order, the correct ground state energy. However, as observed in [9] (for a similar trial state) and later in [17], the energy of the quasi-free state $\widetilde{\Psi}_{N}$ does not match the second-order correction in (1.3). To prove Proposition 1.3, we therefore need to modify the trial state. We do so by replacing the vacuum $\Omega$ in the definition of $\widetilde{\Psi}_{N}$ by the normalised Fock space vector $\xi_{v} /\left\|\xi_{v}\right\|$, with $\xi_{v}=e^{A_{v}} \Omega$ and the cubic phase

$$
A_{v}=\frac{1}{\sqrt{N}} \sum_{\substack{r \in P_{H}, v \in P_{S}: \\ r+v \in P_{H}}} v_{r} \sinh \left(v_{v}\right) a_{r+v}^{*} a_{-r}^{*} a_{-v}^{*} \Theta_{r, v}=\frac{1}{\sqrt{N}} \sum_{\substack{r \in P_{H}, v \in P_{S}: \\ r+v \in P_{H}}} \eta_{r} \sigma_{v} a_{r+v}^{*} a_{-r}^{*} a_{-v}^{*} \Theta_{r, v} .
$$


Here, we introduced the momentum sets

$$
\begin{aligned}
P_{H} & =\left\{p \in \Lambda_{+}^{*}:|p|>N^{1-\kappa-\varepsilon}\right\}, \\
P_{S} & =\left\{p \in \Lambda_{+}^{*}: N^{\kappa / 2-\varepsilon} \leq|p| \leq N^{\kappa / 2+\varepsilon}\right\} .
\end{aligned}
$$

Notice that $P_{S} \subset P_{L}$. On the other hand, to make sure that $P_{H} \cap P_{L}=\emptyset$, from now on we will require that $\varepsilon>0$ is so small that $3 \kappa-2+4 \varepsilon<0$. Moreover, in (2.15) we included, for every $r \in P_{H}$ and every $v \in P_{S}$, the cutoff

$$
\begin{aligned}
\Theta_{r, v}= & \prod_{s \in P_{H}}\left[1-\chi\left(\mathcal{N}_{s}>0\right) \chi\left(\mathcal{N}_{-s+v}>0\right)\right] \\
& \times \prod_{w \in P_{S}}\left[1-\chi\left(\mathcal{N}_{w}>0\right) \chi\left(\mathcal{N}_{r-w}+\mathcal{N}_{-r-v-w}>0\right)\right]
\end{aligned}
$$

where $\mathcal{N}_{p}=a_{p}^{*} a_{p}$ and $\chi(t>0)$ is the indicator function of the set $\{t>0\}$.

Remark. It is easy to check that the computation of the energy and the number of particles of the trial state we are constructing would not change substantially (and would still lead to a proof of Proposition 1.3), if in the definition (2.15) of $A_{v}$ we restricted the sum over $r$ to the finite set $P_{H} \cap\left\{p \in \Lambda_{+}^{*}:|p|<\right.$ $\left.N^{1-\kappa+\varepsilon}\right\}=\left\{p \in \Lambda_{+}^{*}: N^{1-\kappa-\varepsilon}<|p|<N^{1-\kappa+\varepsilon}\right\}$. With this choice, the infinite product over $s \in P_{H}$ appearing in the definition of the cutoff $\Theta_{r, v}$ would be replaced by a finite multiplication.

Let us briefly discuss the action of the cutoff $\Theta_{r, v}$. To understand its role in the computation of $e^{A_{v}} \Omega$, we observe that, for every integer $m \geq 2, r_{1}, \ldots, r_{m} \in P_{H}, v_{1}, \ldots, v_{m} \in P_{S}$, with $r_{1}+v_{1}, \ldots, r_{m}+v_{m} \in$ $P_{H}$, we find

$$
\begin{aligned}
& \Theta_{r_{m}, v_{m}} a_{r_{m-1}+v_{m-1}}^{*} a_{-r_{m-1}}^{*} a_{-v_{m-1}}^{*} \ldots a_{r_{1}+v_{1}}^{*} a_{-r_{1}}^{*} a_{-v_{1}}^{*} \Omega= \\
&=\prod_{i, j=1}^{m-1} \prod_{\substack{p_{\ell} \in\left\{-r_{\ell}, r_{\ell}+v_{\ell}\right\} \\
\ell=i, j, m}} \delta_{p_{i} \neq-p_{j}+v_{m}} \delta_{-p_{m}+v_{i} \neq p_{j}} \\
& \quad \times a_{r_{m-1}+v_{m-1}}^{*} a_{-r_{m-1}}^{*} a_{-v_{m-1}}^{*} \ldots a_{r_{1}+v_{1}}^{*} a_{-r_{1}}^{*} a_{-v_{1}}^{*} \Omega .
\end{aligned}
$$

The choice $i=j$ in the product on the second line introduces restrictions of the form $v_{m} \neq v_{i}$ and $p_{m} \neq p_{i}$ where $p_{\ell} \in\left\{-r_{\ell}, r_{\ell}+v_{\ell}\right\}$ for $\ell=m, i$, for all $i \in\{1, \ldots, m-1\}$ (the condition $p_{i} \neq-p_{i}+v_{m}$, on the other hand, is trivially satisfied due to the assumption $p_{i} \in P_{H}, v_{m} \in P_{S}$ ). For $m \geq 3$, the cutoff $\Theta_{r_{m}, v_{m}}$ implements additional restrictions involving three indices of the form $-p_{i}+v_{j} \neq p_{k}$ with $p_{\ell} \in\left\{-r_{\ell}, r_{\ell}+v_{\ell}\right\}, \ell=i, j, k$ where $i, j, k=1, \ldots, m, i \neq j \neq k$, so that exactly one of the three indices is $m$. We conclude that, for any $m \geq 2$,

$$
\begin{aligned}
A_{v}^{m} \Omega=\frac{1}{N^{m / 2}} \sum_{\substack{r_{1} \in P_{H}, v_{1} \in P_{S}: \\
r_{1}+v_{1} \in P_{H}}} \ldots \sum_{\substack{r_{m} \in P_{H}, v_{m} \in P_{S}: \\
r_{m}+v_{m} \in P_{H}}} \prod_{i=1}^{m} \eta_{r_{i}} \sigma_{v_{i}} \\
\quad \times \theta\left(\left\{r_{j}, v_{j}\right\}_{j=1}^{m}\right) a_{r_{m}+v_{m}}^{*} a_{-r_{m}}^{*} a_{-v_{m}}^{*} \ldots a_{r_{1}+v_{1}}^{*} a_{-r_{1}}^{*} a_{v_{1}}^{*} \Omega
\end{aligned}
$$

where

$$
\theta\left(\left\{r_{j}, v_{j}\right\}_{j=1}^{m}\right)=\prod_{\substack{i, j, k=1 \\ j \neq k}}^{m} \prod_{\substack{p_{i} \in\left\{-r_{i}, r_{i}+v_{i}\right\} \\ p_{k} \in\left\{-r_{k}, r_{k}+v_{k}\right\}}} \delta_{-p_{i}+v_{j} \neq p_{k}}
$$


To illustrate the reason for the introduction of the cutoff, let us compute the norm $\left\|\xi_{v}\right\|$ of the vector $\xi_{v}=e^{A_{\nu}} \Omega$. With (2.17), we find

$$
\begin{aligned}
\left\|\xi_{v}\right\|^{2}= & \sum_{m \geq 0} \frac{1}{(m !)^{2}}\left\|\left(A_{v}\right)^{m} \Omega\right\|^{2} \\
= & \sum_{m \geq 0} \frac{1}{(m !)^{2}} \frac{1}{N^{m}} \sum_{\substack{v_{1}, \tilde{v}_{1} \in P_{S} \\
r_{1}, \tilde{r}_{1} \in P_{H}: \\
r_{1}+v_{1}, \tilde{r}_{1}+\tilde{v}_{1} \in P_{H}}} \ldots \sum_{\begin{array}{c}
v_{m}, \tilde{v}_{m} \in P_{S} \\
r_{m}, \tilde{r}_{m} \in P_{H}: \\
r_{m}+v_{m}, \tilde{r}_{m}+\tilde{v}_{m} \in P_{H}
\end{array}} \theta\left(\left\{\tilde{r}_{j}, \tilde{v}_{j}\right\}_{j=1}^{m}\right) \theta\left(\left\{r_{j}, v_{j}\right\}_{j=1}^{m}\right) \\
& \times \prod_{i=1}^{m} \eta_{r_{i}} \eta_{\tilde{r}_{i}} \sigma_{v_{i}} \sigma_{\tilde{v}_{i}}\left\langle a_{r_{m}+v_{m}}^{*} a_{-r_{m}}^{*} a_{-v_{m}}^{*} \ldots a_{-v_{1}}^{*} \Omega, a_{\tilde{r}_{m}+\tilde{v}_{m}}^{*} a_{-\tilde{r}_{m}}^{*} a_{-\tilde{v}_{m}}^{*} \ldots a_{-\tilde{v}_{1}}^{*} \Omega\right\rangle .
\end{aligned}
$$

Clearly, for the expectation on the last line not to vanish, all creation and annihilation operators with momenta in $P_{S}$ must be contracted among themselves. Since, on the support of $\theta\left(\left\{r_{j}, v_{j}\right\}_{j=1}^{m}\right), v_{i} \neq v_{j}$ for all $i \neq j$ (and, similarly, $\tilde{v}_{i} \neq \tilde{v}_{j}$ for all $i \neq j$ on the support of $\left.\theta\left(\left\{\widetilde{r}_{j}, \widetilde{v}_{j}\right\}_{j=1}^{m}\right)\right)$, we have $(m !)$ identical contributions arising from this pairing. We end up with

$$
\begin{aligned}
\left\|\xi_{v}\right\|^{2}=\sum_{m \geq 0} \frac{1}{m !} \frac{1}{N^{m}} \sum_{\substack{v_{1} \in P_{S}, r_{1}, \tilde{r}_{1} \in P_{H}: \\
r_{1}+v_{1}, \tilde{r}_{1}+v_{1} \in P_{H}}} \ldots \sum_{\substack{v_{m} \in P_{S}, r_{m}, \tilde{r}_{m} \in P_{H}: \\
r_{m}+v_{m}, \tilde{r}_{m}+v_{m} \in P_{H}}} \theta\left(\left\{r_{j}, v_{j}\right\}_{j=1}^{m}\right) \theta\left(\left\{\tilde{r}_{j}, v_{j}\right\}_{j=1}^{m}\right) \\
\times \prod_{i=1}^{m} \eta_{r_{i}} \eta_{\tilde{r}_{i}} \sigma_{v_{i}}^{2}\left\langle\Omega, A_{r_{1}, v_{1}} \ldots A_{r_{m}, v_{m}} A_{\tilde{r}_{m}, v_{m}}^{*} \ldots A_{\tilde{r}_{1}, v_{1}}^{*} \Omega\right\rangle
\end{aligned}
$$

where we have introduced the notation $A_{r_{i}, v_{i}}=a_{r_{i}+v_{i}} a_{-r_{i}}$.

It is now important to observe that, because of the presence of the cutoffs, the annihilation operators in $A_{r_{j}, v_{j}}$ must be contracted with the creation operators in $A_{\widetilde{r}_{j}, v_{j}}$. In fact, if this was not the case, we would have $-r_{j}=-\widetilde{r}_{\ell}$ or $-r_{j}=\widetilde{r}_{\ell}+v_{\ell}$ and $r_{j}+v_{j}=-\widetilde{r}_{k}$ or $r_{j}+v_{j}=\widetilde{r}_{k}+v_{k}$, with at least one of the two indices $\ell, k$ different from $j$. This would imply one of the four relations $\widetilde{r}_{\ell}+v_{j}=-\widetilde{r}_{k}, \widetilde{r}_{\ell}+v_{j}=\widetilde{r}_{k}+v_{k}$, $\widetilde{r}_{\ell}+v_{\ell}=\widetilde{r}_{k}+v_{j},-\widetilde{r}_{\ell}-v_{\ell}+v_{j}=\widetilde{r}_{k}+v_{k}$, all of which are forbidden by the cutoff $\theta\left(\left\{\widetilde{r}_{j}, \widetilde{v}_{j}\right\}_{j=1}^{m}\right)$. We conclude that

$$
\begin{aligned}
\left\langle\Omega, A_{r_{1}, v_{1}} \ldots A_{r_{m}, v_{m}} A_{\tilde{r}_{m}, v_{m}}^{*} \ldots A_{\tilde{r}_{1}, v_{1}}^{*} \Omega\right\rangle \theta\left(\left\{r_{j}, v_{j}\right\}_{j=1}^{m}\right) \theta\left(\left\{\tilde{r}_{j}, \tilde{v}_{j}\right\}_{j=1}^{m}\right) & \\
& =\prod_{i=1}^{m}\left(\delta_{\tilde{r}_{i}, r_{i}}+\delta_{-\tilde{r}_{i}, r_{i}+v_{i}}\right) \theta\left(\left\{r_{j}, v_{j}\right\}_{j=1}^{m}\right)
\end{aligned}
$$

(after identification of the momenta, the second cutoff becomes superfluous). From (2.20), we obtain

$$
\begin{aligned}
\left\|\xi_{v}\right\|^{2} & =\sum_{m \geq 0} \frac{1}{m !} \frac{1}{N^{m}} \sum_{\substack{v_{1} \in P_{S}, r_{1} \in P_{H}: \\
r_{1}+v_{1} \in P_{H}}} \ldots \sum_{\substack{v_{m} \in P_{S}, r_{m} \in P_{H}: \\
r_{m}+v_{m} \in P_{H}}} \theta\left(\left\{r_{j}, v_{j}\right\}_{j=1}^{m}\right) \prod_{i=1}^{m} \eta_{r_{i}}\left(\eta_{r_{i}}+\eta_{r_{i}+v_{i}}\right) \sigma_{v_{i}}^{2} \\
& =\sum_{m \geq 0} \frac{1}{2^{m} m !} \frac{1}{N^{m}} \sum_{\substack{v_{1} \in P_{S}, r_{1} \in P_{H}: \\
r_{1}+v_{1} \in P_{H}}} \ldots \sum_{\substack{v_{m} \in P_{S}, r_{m} \in P_{H}: \\
r_{m}+v_{m} \in P_{H}}} \theta\left(\left\{r_{j}, v_{j}\right\}_{j=1}^{m}\right) \prod_{i=1}^{m}\left(\eta_{r_{i}}+\eta_{r_{i}+v_{i}}\right)^{2} \sigma_{v_{i}}^{2}
\end{aligned}
$$

where we used the invariance of $\theta$, with respect to $-r_{i} \rightarrow r_{i}+v_{i}$. The cutoffs have been used first to exclude coinciding momenta in $v_{1}, \ldots, v_{m}$ and in $\widetilde{v}_{1}, \ldots, \widetilde{v}_{m}$ (which implies that, up to permutations, the pairing of the momenta in $P_{S}$ is unique) and then in (2.21) to make sure that annihilation operators in $A_{r_{j}, v_{j}}$ can only be contracted with the creation operators in $A_{\widetilde{r}_{j}, v_{j}}^{*}$. This substantially simplifies computations. Similar simplifications will arise in the computation of the energy of our trial state. 
Apart from the formula (2.22) for the norm $\left\|\xi_{v}\right\|^{2}$, we will also need bounds on the expectation, in the state $\xi_{v} /\left\|\xi_{v}\right\|$, of the number of particles operator $\mathcal{N}$, of $\mathcal{N}^{2}$, of the kinetic energy operator $\mathcal{K}$ and of the product $\mathcal{K N}$. These bounds are collected in the next proposition, whose proof will be discussed in Section 5 .

Proposition 2.2. Let $\xi_{v}=e^{A_{\nu}} \Omega$ with $e^{A_{v}}$ defined in (2.15) with $\kappa \in(1 / 2 ; 2 / 3)$ and $\varepsilon>0$ such that $3 \kappa-2+4 \varepsilon<0$. Then, under the assumptions of Theorem 1.1, we have

$$
\frac{\left\langle\xi_{v}, \mathcal{N}^{j} \xi_{v}\right\rangle}{\left\|\xi_{v}\right\|^{2}} \leq C N^{(9 \kappa / 2-2+\varepsilon) j}
$$

and

$$
\frac{\left\langle\xi_{\nu}, \mathcal{K N}^{j-1} \xi_{\nu}\right\rangle}{\left\|\xi_{\nu}\right\|^{2}} \leq C N^{5 \kappa / 2} N^{(9 \kappa / 2-2+\varepsilon)(j-1)}
$$

for $j=1,2$.

Using the Weyl operator $W_{N_{0}}$ from (2.3), the Bogoliubov transformation $T_{v}$ defined in (2.13) and the cubic phase $A_{v}$ introduced in (2.15) (or, equivalently, the vector $\xi_{v}=e^{A_{v}} \Omega$ ), we can now define our trial state

$$
\Psi_{N}=\frac{W_{N_{0}} T_{v} e^{A_{\nu}} \Omega}{\left\|W_{N_{0}} T_{v} e^{A_{\nu}} \Omega\right\|}=W_{N_{0}} T_{v} \frac{\xi_{v}}{\left\|\xi_{v}\right\|} .
$$

Here, we choose $N_{0}>0$ such that

$$
N=N_{0}+\left\|\sigma_{L}\right\|^{2}
$$

where $\sigma_{L}$ denotes the restriction to the set $P_{L}$ of the coefficients $\sigma_{p}=\sinh \left(v_{p}\right)$, with $v_{p}$ defining the Bogoliubov transformation $T_{v}$; see (2.13).

Let us briefly compare our trial state with the one of [20]. In both approaches, the condensate is perturbed with operators creating double and triple excitations, the latter having two particles with high momenta and one particle with low momentum. Moreover, similarly as in [20], we impose cutoffs making sure that each low momentum appears only once. In contrast to [20], we also impose cutoffs on high momenta. Moreover, we have a clearer separation between creation of pairs (obtained through the Bogoliubov transformation $T_{v}$ ) and creation of triples. Finally, in our approach, we create triple excitations through the action of $e^{A_{\nu}}$ on the vacuum; the algebraic structure of the exponential makes the analysis and the combinatorics much simpler.

As shown in the next proposition, the choice (2.26) of $N_{0}$ guarantees that $\Psi_{N}$ has the expected number of particles.

Proposition 2.3. Let $\Psi_{N}$ be defined in (2.25) with the parameter $N_{0}$ appearing in (2.3) defined by (2.26). Let $\kappa \in(1 / 2 ; 2 / 3)$ and $\varepsilon>0$ so that $3 \kappa-2+4 \varepsilon<0$. Then

$$
\left\langle\Psi_{N}, \mathcal{N} \Psi_{N}\right\rangle \geq N, \quad\left\langle\Psi_{N}, \mathcal{N}^{2} \Psi_{N}\right\rangle \leq C N^{2}
$$

for all $N$ large enough.

To prove Proposition 2.3 (and later to show other properties of the trial state $\Psi_{N}$ ) in the next lemma we collect some bounds for norms of the coefficients appearing in the definition of $A_{v}$ in (2.15). We denote here by $\eta_{L}, \eta_{L^{c}}, \eta_{S}, \eta_{H}$ the restriction of $\eta: \Lambda^{*} \rightarrow \mathbb{R}$ to the set $P_{L}, P_{L}^{c}, P_{S}$ and, respectively, $P_{H}$. Similarly, we define $\gamma_{L}, \gamma_{L^{c}}, \gamma_{H}, \gamma_{S}$ and $\sigma_{L}, \sigma_{L^{c}}, \sigma_{H}, \sigma_{S}$. 
Lemma 2.4. We have

$$
\begin{aligned}
& \left\|\eta_{L^{c}}\right\|^{2} \leq C N^{3 \kappa / 2-\varepsilon}, \quad\left\|\eta_{L^{c}}\right\|_{H^{1}}^{2} \leq C N^{1+\kappa}, \quad\left\|\eta_{L^{c}}\right\|_{\infty} \leq C N^{-2 \varepsilon} \\
& \left\|\eta_{H}\right\|^{2} \leq C N^{3 \kappa-1+\varepsilon}, \quad\left\|\eta_{H}\right\|_{H^{1}}^{2} \leq C N^{1+\kappa}, \quad\left\|\eta_{H}\right\|_{\infty} \leq C N^{3 \kappa-2+2 \varepsilon} \text {. }
\end{aligned}
$$

In particular, this implies that $\left\|\gamma_{H}\right\|_{\infty},\left\|\sigma_{H}\right\|_{\infty} \leq C$. Moreover, we have

$$
\left\|\gamma_{L}\right\|_{\infty}^{2},\left\|\sigma_{L}\right\|_{\infty}^{2} \leq C N^{\kappa / 2}, \quad\left\|\gamma_{L} \sigma_{L}\right\|_{1} \leq C N^{3 \kappa / 2+\varepsilon}
$$

and

$$
\left\|\gamma_{L}\right\|^{2} \leq C N^{3 \kappa / 2+3 \varepsilon}, \quad\left\|\sigma_{L}\right\|^{2} \leq C N^{3 \kappa / 2}, \quad\left\|\sigma_{L}\right\|_{H^{1}}^{2} \leq C N^{5 \kappa / 2+\varepsilon} .
$$

Finally, we observe that

$$
\left\|\sigma_{S}\right\|^{2} \leq C N^{3 \kappa / 2}, \quad\left\|\sigma_{S}\right\|_{H^{1}}^{2} \leq C N^{5 \kappa / 2+\varepsilon}, \quad\left\|\gamma_{S}\right\|_{\infty}^{2},\left\|\sigma_{S}\right\|_{\infty}^{2} \leq C N^{\varepsilon} .
$$

Proof. The bounds for $\left\|\eta_{L^{c}}\right\|,\left\|\eta_{H}\right\|,\left\|\eta_{L^{c}}\right\|_{\infty}$ and $\left\|\eta_{H}\right\|_{\infty}$ follow from (2.8). On the other hand, with the notation $\breve{\eta}(x)=-N w_{\ell}\left(N^{1-\kappa} x\right)$ for the function on $\Lambda$ with Fourier coefficients $\eta_{p}$, we find from Lemma 2.1, part iii),

$$
\left\|\eta_{L^{c}}\right\|_{H^{1}}^{2} \leq C \sum_{p \in \Lambda^{*}} p^{2}\left|\eta_{p}\right|^{2} \leq C \int|\nabla \check{\eta}(x)|^{2} d x \leq C N^{1+\kappa} \int_{\mathbb{R}^{3}} \frac{1}{\left(|x|^{2}+1\right)^{2}} d x \leq C N^{1+\kappa} .
$$

To show bounds for $\sigma_{L}, \gamma_{L}$ we observe that, with (2.12) and $\gamma_{p}^{2}=1+\sigma_{p}^{2}$, we obtain

$$
\sigma_{p}^{2}=\frac{p^{2}+8 \pi \mathfrak{a} N^{\kappa}-\sqrt{|p|^{4}+16 \pi \mathfrak{a} N^{\kappa} p^{2}}}{2 \sqrt{|p|^{4}+16 \pi \mathfrak{a} N^{\kappa} p^{2}}}, \quad \sigma_{p} \gamma_{p}=\frac{-8 \pi \mathfrak{a} N^{\kappa}}{2 \sqrt{|p|^{4}+16 \pi \mathfrak{a} N^{\kappa} p^{2}}} .
$$

Recalling that $P_{L}=\left\{p \in \Lambda_{+}^{*}:|p| \leq N^{\kappa / 2+\varepsilon}\right\}$, we find

$$
\left\|\sigma_{L}\right\|_{\infty}^{2} \leq \sup _{p \in P_{L}:|p| \leq N^{\kappa / 2}} C \frac{N^{\kappa / 2}}{|p|}+\sup _{p \in P_{L}:|p| \geq N^{\kappa / 2}} C \frac{N^{2 \kappa}}{|p|^{4}} \leq C N^{\kappa / 2} .
$$

Moreover, by definition of $P_{S}$ we get

$$
\left\|\sigma_{S}\right\|_{\infty}^{2} \leq \sup _{N^{\kappa / 2-\varepsilon} \leq|p| \leq N^{\kappa / 2}} C \frac{N^{\kappa / 2}}{|p|}+\sup _{p \in P_{L}:|p| \geq N^{\kappa / 2}} C \frac{N^{2 \kappa}}{|p|^{4}} \leq C N^{\varepsilon} .
$$

Using again $\gamma_{p}^{2}=1+\sigma_{p}^{2}$, we find $\left\|\gamma_{L}\right\|_{\infty} \leq C N^{\kappa / 2}$ and $\left\|\gamma_{S}\right\|_{\infty} \leq C N^{\varepsilon}$. Similarly, we obtain

$$
\left\|\sigma_{L}\right\|^{2} \leq C \sum_{p \in P_{L}:|p| \leq N^{\kappa / 2}} \frac{N^{\kappa / 2}}{|p|}+C \sum_{p \in P_{L}:|p|>N^{\kappa / 2}} \frac{N^{2 \kappa}}{|p|^{4}} \leq C N^{3 \kappa / 2}
$$

and thus $\left\|\gamma_{L}\right\|^{2} \leq C N^{3 \kappa / 2+3 \varepsilon}$. Moreover, we have

$$
\left\|\sigma_{L}\right\|_{H_{1}}^{2} \leq C \sum_{p \in P_{L}:|p| \leq N^{\kappa / 2}}|p| N^{\kappa / 2}+C \sum_{p \in P_{L}:|p| \geq N^{\kappa / 2}} \frac{N^{2 \kappa}}{p^{2}} \leq C N^{5 \kappa / 2+\varepsilon} .
$$


The bound for $\left\|\sigma_{L} \gamma_{L}\right\|_{1}$ is proved similarly, using the expression for $\gamma_{p} \sigma_{p}$ in (2.28). Finally, we note that the estimates (2.30) and (2.31) do not improve when we consider the restriction of $\sigma_{p}$ to $P_{S} \subset P_{L}$; hence, $\left\|\sigma_{S}\right\|^{2} \leq C N^{3 \kappa / 2}$ and $\left\|\sigma_{S}\right\|_{H^{1}}^{2} \leq C N^{5 \kappa / 2+\varepsilon}$.

We can now return to the proof of Proposition 2.3.

Proof of Proposition 2.3. From (2.4) and (2.14) we get

$$
\begin{aligned}
T_{\nu}^{*} W_{N_{0}}^{*} \mathcal{N} W_{N_{0}} T_{v}= & N_{0}+\sum_{p \in \Lambda_{+}^{*}} \sigma_{p}^{2}+\sqrt{N_{0}}\left(a_{0}+a_{0}^{*}\right)+a_{0}^{*} a_{0} \\
& +\sum_{p \in \Lambda_{+}^{*}}\left[\left(\sigma_{p}^{2}+\gamma_{p}^{2}\right) a_{p}^{*} a_{p}+\gamma_{p} \sigma_{p}\left(a_{p} a_{-p}+\text { h.c. }\right)\right]
\end{aligned}
$$

By definition of $\xi_{v}, a_{0} \xi_{v}=0$ and $\left\langle\xi_{v}, a_{p} a_{-p} \xi_{v}\right\rangle=0$ for every $p \in \Lambda^{*}$, as well as the definition $N=N_{0}+\left\|\sigma_{L}\right\|^{2}$, we obtain that

$$
\left\langle\Psi_{N}, \mathcal{N} \Psi_{N}\right\rangle=\frac{\left\langle\xi_{v}, T_{v}^{*} W_{N_{0}}^{*} \mathcal{N} W_{N_{0}} T_{\nu} \xi_{v}\right\rangle}{\left\|\xi_{v}\right\|^{2}}=N+\sum_{p \in P_{L}^{c}} \sigma_{p}^{2}+\sum_{p \in P_{S} \cup P_{H}}\left(\sigma_{p}^{2}+\gamma_{p}^{2}\right) \frac{\left\langle\xi_{v}, a_{p}^{*} a_{p} \xi_{v}\right\rangle}{\left\|\xi_{v}\right\|^{2}} .
$$

This immediately implies that $\left\langle\Psi_{N}, \mathcal{N} \Psi_{N}\right\rangle \geq N$.

With $a_{0} \xi_{v}=0$ and the assumption $3 \kappa-2+4 \varepsilon<0,(2.32)$ also implies that

$$
\begin{aligned}
\left\langle\xi_{v}, T_{\nu}^{*} W_{N_{0}}^{*} \mathcal{N}^{2} W_{N_{0}} T_{\nu} \xi_{v}\right\rangle \leq & C N^{2}+C \sum_{p, q \in \Lambda_{+}^{*}}\left(\sigma_{p}^{2}+\gamma_{p}^{2}\right)\left(\sigma_{q}^{2}+\gamma_{q}^{2}\right)\left\langle\xi_{v}, a_{p}^{*} a_{p} a_{q}^{*} a_{q} \xi_{v}\right\rangle \\
& +C \sum_{p, q \in \Lambda_{+}^{*}} \gamma_{p} \sigma_{p} \gamma_{q} \sigma_{q}\left\langle\xi_{v},\left(a_{p} a_{-p}+a_{p}^{*} a_{-p}^{*}\right)\left(a_{q} a_{-q}+a_{q}^{*} a_{-q}^{*}\right) \xi_{v}\right\rangle .
\end{aligned}
$$

Since $\xi_{v}$ is a superposition of states with $3 m$ particles with momenta in $P_{H} \cup P_{S}$, we obtain, writing $a_{p} a_{-p} a_{q}^{*} a_{-q}^{*}=a_{q}^{*} a_{p} a_{-q}^{*} a_{-p}+\left(\delta_{p, q}+\delta_{-p, q}\right)\left(a_{p}^{*} a_{p}+1\right)+\delta_{p, q} a_{-p}^{*} a_{-p}$ and similarly for $a_{p}^{*} a_{-p}^{*} a_{q} a_{-q}$,

$$
\begin{aligned}
\left\langle\xi_{v}, T_{v}^{*} W_{N_{0}}^{*} \mathcal{N}^{2} W_{N_{0}} T_{\nu} \xi_{v}\right\rangle \leq & C N^{2}+C N^{2 \varepsilon}\left\langle\xi_{v},(\mathcal{N}+1)^{2} \xi_{v}\right\rangle+C \sum_{p \in P_{H} \cup P_{S}} \gamma_{p}^{2} \sigma_{p}^{2}\left\langle\xi_{v}, a_{p}^{*} a_{p} \xi_{v}\right\rangle \\
& +C \sum_{p \in \Lambda_{+}^{*}} \gamma_{p}^{2} \sigma_{p}^{2}\left\|\xi_{v}\right\|^{2}+C \sum_{p, q \in P_{H} \cup P_{S}} \gamma_{p} \sigma_{p} \gamma_{q} \sigma_{q}\left\langle\xi_{v}, a_{p}^{*} a_{q} a_{-p}^{*} a_{-q} \xi_{v}\right\rangle
\end{aligned}
$$

Estimating the last term through

$$
\begin{aligned}
&\left|\sum_{p, q \in P_{H} \cup P_{S}} \gamma_{p} \sigma_{p} \gamma_{q} \sigma_{q}\left\langle\xi_{v}, a_{p}^{*} a_{q} a_{-p}^{*} a_{-q} \xi_{v}\right\rangle\right| \\
& \leq \sum_{p, q \in P_{H} \cup P_{S}}\left|\gamma_{p}\left\|\sigma_{p}\right\| \gamma_{q}\left\|\sigma_{q} \mid\right\| a_{q}^{*} a_{p} \xi_{v}\|\| a_{-p}^{*} a_{-q} \xi_{v} \|\right. \\
& \leq \sum_{p, q \in P_{H} \cup P_{S}}\left|\gamma_{p}\left\|\sigma_{p}\right\| \gamma_{q} \| \sigma_{q}\right|\left[\left\|a_{q} a_{p} \xi_{v}\right\|+\left\|a_{p} \xi_{v}\right\|\right]\left[\left\|a_{-p} a_{-q} \xi_{v}\right\|+\left\|a_{-q} \xi_{v}\right\|\right] \\
& \leq C\left\|\gamma_{S \cup H}\right\|_{\infty}^{2}\left\|\sigma_{S \cup H}\right\|_{\infty}^{2}\left\|(\mathcal{N}+1) \xi_{v}\right\|^{2}+C\left\|\gamma_{S \cup H}\right\|_{\infty}^{2}\left\|\sigma_{S \cup H}\right\|^{2}\left\|\mathcal{N}^{1 / 2} \xi_{v}\right\|^{2} \\
&+C\left\|\gamma_{S \cup H}\right\|_{\infty}^{2}\left\|\sigma_{S \cup H}\right\|_{\infty}\left\|\sigma_{S \cup H}\right\|\left\|(\mathcal{N}+1) \xi_{v}\right\|\left\|\mathcal{N}^{1 / 2} \xi_{v}\right\|,
\end{aligned}
$$

we conclude with the bounds in Lemma 2.4 and in Proposition 2.2 that, for $3 \kappa-2+4 \varepsilon<0$,

$$
\begin{aligned}
\left\langle\xi_{v}, T_{\nu}^{*} W_{N_{0}}^{*} \mathcal{N}^{2} W_{N_{0}} T_{\nu} \xi_{v}\right\rangle & \leq C N^{2}+C N^{2 \varepsilon}\left\langle\xi_{v}, \mathcal{N}^{2} \xi_{v}\right\rangle+C N^{\varepsilon}\|\sigma\|^{2}\left\langle\xi_{v}, \mathcal{N} \xi_{v}\right\rangle+C\|\gamma\|_{\infty}^{2}\|\sigma\|^{2} \\
& \leq C N^{2}+C N^{9 \kappa-4+4 \varepsilon}+C N^{6 \kappa-2+2 \varepsilon}+C N^{2 \kappa} \leq C N^{2}
\end{aligned}
$$


The next theorem, whose proof occupies the rest of the article, determines the energy of $\Psi_{N}$ and, combined with Proposition 2.3, allows us to conclude the proof of Proposition 1.3.

Theorem 2.5. Let $\mathcal{H}_{N}$ and $\Psi_{N} \in \mathcal{F}$ be defined as in (2.2) and (2.25), respectively, and let $E_{N}^{\Psi}=$ $\left\langle\Psi_{N}, \mathcal{H}_{N} \Psi_{N}\right\rangle$. Let $\kappa \in(1 / 2 ; 2 / 3), \varepsilon>0$ so small that $3 \kappa-2+4 \varepsilon<0$. Then, under the assumption of Theorem 1.1, we have

$$
E_{N}^{\Psi} \leq 4 \pi \mathfrak{a} N^{1+\kappa}\left(1+\frac{128}{15 \sqrt{\pi}}\left(\mathfrak{a}^{3} N^{3 \kappa-2}\right)^{1 / 2}\right)+C N^{5 \kappa / 2} \max \left\{N^{-\varepsilon}, N^{9 \kappa-5+6 \varepsilon}, N^{21 \kappa / 4-3+3 \varepsilon}\right\}
$$

for all $N$ large enough.

Remark. Equation (2.34) gives the correct second-order term for all $\kappa<5 / 9$ (choosing $\varepsilon>0$ small enough); this corresponds to exponents $\gamma<4 / 3$ in Proposition 1.3. With a more complicated proof, we could have considered all $\kappa<7 / 12$ (corresponding to $\gamma<5 / 3$ ).

Proof of Proposition 1.3. Proposition 1.3 follows from Proposition 2.3 and Theorem 2.5, recalling that (1.5) is unitarily equivalent to $L^{-2} \mathcal{H}_{N}$, with $\mathcal{H}_{N}$ as defined in (2.2), $L=\tilde{\rho}^{-\gamma}, N=\tilde{\rho} L^{3}=\tilde{\rho}^{1-3 \gamma}$ and $\kappa=(2 \gamma-1) /(3 \gamma-1)$. At the end, to obtain (1.8) and (1.9), we have to rename $\varepsilon(3 \gamma-1) \rightarrow \varepsilon$.

\section{Energy of the trial state}

In this section we prove Theorem 2.5. With (2.25) and introducing the notations

$$
\mathcal{G}_{N}=T_{\nu}^{*} \mathcal{L}_{N} T_{\nu}, \quad \text { with } \quad \mathcal{L}_{N}=W_{N_{0}}^{*} \mathcal{H}_{N} W_{N_{0}}
$$

we write

$$
E_{N}^{\Psi}=\left\langle\Psi_{N}, \mathcal{H}_{N} \Psi_{N}\right\rangle=\frac{\left\langle\xi_{v}, \mathcal{G}_{N} \xi_{v}\right\rangle}{\left\|\xi_{v}\right\|^{2}}
$$

with $\xi_{v}=e^{A_{v}} \Omega$, as defined before (2.15). With (2.2) and recalling from (2.4) that

$$
W_{N_{0}}^{*} a_{p} W_{N_{0}}=a_{p}+\sqrt{N_{0}} \delta_{p, 0}
$$

we obtain $\mathcal{L}_{N}=\mathcal{L}_{N}^{(0)}+\mathcal{L}_{N}^{(1)}+\mathcal{L}_{N}^{(2)}+\mathcal{L}_{N}^{(3)}+\mathcal{L}_{N}^{(4)}$, with

$$
\begin{aligned}
\mathcal{L}_{N}^{(0)}= & \frac{N_{0}^{2}}{2} N^{\kappa-1} \widehat{V}(0) \\
\mathcal{L}_{N}^{(1)}= & N_{0}^{3 / 2} N^{\kappa-1} \widehat{V}(0)\left(a_{0}+\text { h.c. }\right) \\
\mathcal{L}_{N}^{(2)}= & \sum_{p \in \Lambda^{*}} p^{2} a_{p}^{*} a_{p}+\frac{N_{0}}{N} \sum_{p \in \Lambda^{*}} N^{\kappa}\left(\widehat{V}\left(p / N^{1-\kappa}\right)+\widehat{V}(0)\right) a_{p}^{*} a_{p} \\
& \quad+\frac{N_{0}}{2 N} \sum_{p \in \Lambda^{*}} N^{\kappa} \widehat{V}\left(p / N^{1-\kappa}\right)\left(a_{p}^{*} a_{-p}^{*}+a_{p} a_{-p}\right) \\
\mathcal{L}_{N}^{(3)}= & \frac{\sqrt{N_{0}}}{N} \sum_{p, r \in \Lambda^{*}} N^{\kappa} \widehat{V}\left(r / N^{1-\kappa}\right)\left(a_{p}^{*} a_{r}^{*} a_{p+r}+a_{p+r}^{*} a_{r} a_{p}\right) \\
\mathcal{L}_{N}^{(4)}= & \frac{1}{2 N} \sum_{p, q, r \in \Lambda^{*}} N^{\kappa} \widehat{V}\left(r / N^{1-\kappa}\right) a_{p+r}^{*} a_{q}^{*} a_{p} a_{q+r} .
\end{aligned}
$$

To compute $\mathcal{G}_{N}$, we have to conjugate the operators in (3.3) with the Bogoliubov transformation $T_{\nu}$. The result is described in the following proposition, whose proof will be discussed in Section 4 . 
Proposition 3.1. Let

$$
\mathcal{K}=\sum_{p \in \Lambda_{+}^{*}} p^{2} a_{p}^{*} a_{p}, \quad \mathcal{V}_{N}^{(H)}=\frac{1}{2 N} \sum_{\substack{r \in \Lambda^{*}, p, q \in P_{H}: \\ p+r, q+r \in P_{H}}} N^{\kappa} \widehat{V}\left(r / N^{1-\kappa}\right) a_{p+r}^{*} a_{q}^{*} a_{p} a_{q+r}
$$

and (recalling from (2.26) that $N_{0}=N-\left\|\sigma_{L}\right\|^{2}$ )

$$
\mathcal{C}_{N}=\frac{\sqrt{N_{0}}}{N} \sum_{\substack{p, r \in P_{H} \\ p+r \in P_{S}}} N^{\kappa} \widehat{V}\left(r / N^{1-\kappa}\right) \sigma_{p+r} \gamma_{p} \gamma_{r}\left(a_{p+r}^{*} a_{-p}^{*} a_{-r}^{*}+\text { h.c. }\right)
$$

Moreover, let

$$
\begin{aligned}
C_{\mathcal{G}_{N}}= & \frac{N^{1+\kappa}}{2} \widehat{V}(0)+\sum_{p \in \Lambda_{+}^{*}} p^{2} \sigma_{p}^{2}+\sum_{p \in \Lambda_{+}^{*}} N^{\kappa} \widehat{V}\left(p / N^{1-\kappa}\right) \sigma_{p} \gamma_{p} \\
& +\sum_{p \in P_{L}} N^{\kappa} \widehat{V}\left(p / N^{1-\kappa}\right) \sigma_{p}^{2}+\frac{1}{2 N} \sum_{\substack{p, r \in \Lambda_{+}^{*} \\
r \neq p}} N^{\kappa} \widehat{V}\left(r / N^{1-\kappa}\right) \sigma_{p} \sigma_{p-r} \gamma_{p} \gamma_{p-r} \\
& -\frac{1}{N} \sum_{v \in P_{L}} \sigma_{v}^{2} \sum_{p \in P_{L}^{c}} N^{\kappa} \widehat{V}\left(p / N^{1-\kappa}\right) \eta_{p} .
\end{aligned}
$$

Then, under the assumptions of Theorem 1.1, for all $\kappa \in(1 / 2 ; 2 / 3)$ and $\varepsilon>0$ with $3 \kappa-2+4 \varepsilon<0$ and $N$ sufficiently large, we have

$$
\begin{aligned}
\frac{\left\langle\xi_{v}, \mathcal{G}_{N} \xi_{v}\right\rangle}{\left\|\xi_{v}\right\|^{2}} \leq & C_{\mathcal{G}_{N}}+\frac{\left\langle\xi_{v},\left(\mathcal{K}+\mathcal{V}_{N}^{(H)}+\mathcal{C}_{N}\right) \xi_{v}\right\rangle}{\left\|\xi_{v}\right\|^{2}} \\
& +C N^{5 \kappa / 2} \cdot \max \left\{N^{-\varepsilon}, N^{9 \kappa-5+6 \varepsilon}, N^{21 \kappa / 4-3+3 \varepsilon}\right\} .
\end{aligned}
$$

Remark. In fact, (3.7) does not only hold true for $\xi_{v}$ but for any state satisfying the bounds in Proposition 2.2.

The expectation of the operators $\mathcal{K}, \mathcal{V}_{N}^{(H)}, \mathcal{C}_{N}$ in the state $\xi_{v} /\left\|\xi_{v}\right\|$, appearing on the right-hand side of (3.7) is determined by the next proposition, which will be shown in Section 5.

Proposition 3.2. Under the assumptions of Theorem 1.1, we have

$$
\begin{aligned}
\frac{\left\langle\xi_{v},\left(\mathcal{K}+\mathcal{V}_{N}^{(H)}+\mathcal{C}_{N}\right) \xi_{v}\right\rangle}{\left\|\xi_{v}\right\|^{2}} \leq & \frac{1}{N} \sum_{v \in P_{L}} \sigma_{v}^{2} \sum_{r \in P_{H}} N^{\kappa} \widehat{V}\left(r / N^{1-\kappa}\right)\left(\eta_{r}+\eta_{r+v}\right) \\
& +C N^{5 \kappa / 2} \cdot \max \left\{N^{-\varepsilon}, N^{12 \kappa-7+5 \varepsilon}\right\}
\end{aligned}
$$

for all $\kappa \in(1 / 2 ; 2 / 3)$ and all $\varepsilon>0$ so small that $3 \kappa-2+4 \varepsilon<0$. 
Let us now use the statements of Proposition 3.1 and Proposition 3.2 to obtain an upper bound for the energy of the trial state $\Psi_{N}$ and prove Theorem 2.5. From Proposition 3.1 and Proposition 3.2 we find

$$
\begin{aligned}
E_{N}^{\Psi} \leq & \frac{N^{1+\kappa}}{2} \widehat{V}(0)+\sum_{p \in \Lambda_{+}^{*}} p^{2} \sigma_{p}^{2}+\sum_{p \in \Lambda_{+}^{*}} N^{\kappa} \widehat{V}\left(p / N^{1-\kappa}\right) \gamma_{p} \sigma_{p} \\
& +\sum_{v \in P_{L}} N^{\kappa} \widehat{V}\left(v / N^{1-\kappa}\right) \sigma_{v}^{2}+\frac{1}{2 N} \sum_{\substack{p, q \in \Lambda_{+}^{*} \\
p \neq q}} N^{\kappa} \widehat{V}\left((p-q) / N^{1-\kappa}\right) \gamma_{p} \gamma_{q} \sigma_{p} \sigma_{q} \\
& -\frac{1}{N} \sum_{v \in P_{L}} \sigma_{v}^{2} \sum_{p \in P_{L}^{c}} N^{\kappa} \widehat{V}\left(p / N^{1-\kappa}\right) \eta_{p} \\
& +\frac{1}{N} \sum_{v \in P_{L}} \sigma_{v}^{2} \sum_{r \in P_{H}} N^{\kappa} \widehat{V}\left(r / N^{1-\kappa}\right)\left(\eta_{r}+\eta_{r+v}\right)+\mathcal{E}
\end{aligned}
$$

with

$$
\mathcal{E} \leq C N^{5 \kappa / 2} \cdot \max \left\{N^{-\varepsilon}, N^{9 \kappa-5+6 \varepsilon}, N^{21 \kappa / 4-3+3 \varepsilon}\right\}
$$

for all $\kappa \in(1 / 2 ; 2 / 3)$ and all $\varepsilon>0$ with $3 \kappa-2+4 \varepsilon<0$ (in this range, $12 \kappa-7+5 \varepsilon<9 \kappa-5+3 \varepsilon$ ). Since $\left|\sigma_{p}-\eta_{p}\right| \leq C\left|\eta_{p}\right|^{3}$ for all $p \in P_{L}^{c}$, with (2.8) we find

$$
\sum_{p \in \Lambda_{+}^{*}} p^{2} \sigma_{p}^{2} \leq \sum_{v \in P_{L}} v^{2} \sigma_{v}^{2}+\sum_{p \in P_{L}^{c}} p^{2} \eta_{p}^{2}+C N^{5 \kappa / 2-3 \varepsilon} .
$$

Similarly, with $\left|\gamma_{p} \sigma_{p}-\eta_{p}\right| \leq C \eta_{p}^{3}$ for all $p \in P_{L}^{c}$, the last term on the first line of (3.8) can be written as

$$
\begin{aligned}
\sum_{p \in \Lambda_{+}^{*}} N^{\kappa} & \widehat{V}\left(p / N^{1-\kappa}\right) \gamma_{p} \sigma_{p} \\
& \leq \sum_{v \in P_{L}} N^{\kappa} \widehat{V}\left(v / N^{1-\kappa}\right) \gamma_{v} \sigma_{v}+\sum_{p \in P_{L}^{c}} N^{\kappa} \widehat{V}\left(p / N^{1-\kappa}\right) \eta_{p}+C N^{5 \kappa / 2-3 \varepsilon}
\end{aligned}
$$

Next, we focus on the last term on the second line of (3.8). We define $\mathcal{E}_{1}$ through the identity

$$
\begin{aligned}
& \frac{1}{2 N} \sum_{\substack{p, q \in \Lambda_{+}^{*} \\
p \neq q}} N^{\kappa} \widehat{V}\left((p-q) / N^{1-\kappa}\right) \gamma_{p} \gamma_{q} \sigma_{p} \sigma_{q} \\
& =\frac{1}{N} \sum_{\substack{p \in P_{L}^{c}, q \in P_{L}}} N^{\kappa} \widehat{V}\left((p-q) / N^{1-\kappa}\right) \eta_{p} \gamma_{q} \sigma_{q}+\frac{1}{2 N} \sum_{\substack{p, q \in P_{L}^{c} \\
p \neq q}} N^{\kappa} \widehat{V}\left((p-q) / N^{1-\kappa}\right) \eta_{p} \eta_{q}+\mathcal{E}_{1}
\end{aligned}
$$

Using again $\left|\gamma_{p} \sigma_{p}-\eta_{p}\right| \leq C\left|\eta_{p}\right|^{3}$ for all $p \in P_{L}^{c}$, the estimate

$$
\sup _{r \in \Lambda^{*}} \sum_{s \in \Lambda_{+}^{*}} N^{\kappa}\left|\widehat{V}\left((r-s) / N^{1-\kappa}\right) \| \eta_{s}\right| \leq C N^{1+\kappa}
$$

and the bounds from Lemma 2.4, we conclude (using the condition $3 \kappa-2+5 \varepsilon<0$ ) that

$$
\mathcal{E}_{1} \leq C\left[N^{\kappa}\left\|\eta_{L^{c}}\right\|_{\infty}\left\|\eta_{L^{c}}\right\|^{2}+N^{\kappa-1}\left\|\eta_{L^{c}}\right\|_{\infty}\left\|\eta_{L^{c}}\right\|^{2}\left\|\sigma_{L} \gamma_{L}\right\|_{1}+N^{\kappa-1}\left\|\sigma_{L} \gamma_{L}\right\|_{1}^{2}\right] \leq C N^{5 \kappa / 2-\varepsilon}
$$


To prove (3.12) we use (2.8) and we remark that

$$
\sum_{s \in \Lambda^{*}:|s| \leq N^{1-\kappa}} N^{\kappa}\left|\widehat{V}\left((r-s) / N^{1-\kappa}\right)\right|\left|\eta_{s}\right| \leq C N^{2 \kappa} \sum_{s \in \Lambda_{+}^{*}:|s| \leq N^{1-\kappa}}|s|^{-2} \leq C N^{1+\kappa}
$$

and that, rescaling variables (setting $\tilde{r}=r / N^{1-\kappa}$ ) and using an integral approximation,

$$
\begin{aligned}
\sum_{s \in \Lambda_{+}^{*}:|s|>N^{1-\kappa}} N^{\kappa}\left|\widehat{V}\left((r-s) / N^{1-\kappa}\right)\right|\left|\eta_{s}\right| & \leq C N^{1+\kappa} \sum_{s \in \Lambda^{*} / N^{1-\kappa}:|s|>1} N^{-3(1-\kappa)}|\widehat{V}(\tilde{r}-s) \| s|^{-2} \\
& \leq C N^{1+\kappa} \int_{|s|>1}\left|\widehat{V}(\tilde{r}-s)\left\|\left.s\right|^{-2} d s \leq C_{q} N^{1+\kappa}\right\| \widehat{V} \|_{q}\right.
\end{aligned}
$$

for any $q<3$. With the assumption $V \in L^{q^{\prime}}\left(\mathbb{R}^{3}\right)$, for some $q^{\prime}>3 / 2$, (3.12) follows by the HausdorffYoung inequality.

Finally, we remark that the terms in the last two lines of (3.8) can be combined, using (2.8) and the bound $\left\|\sigma_{L}\right\|^{2} \leq C N^{3 \kappa / 2}$ from Lemma 2.4, into

$$
\begin{aligned}
-\frac{1}{N} \sum_{v \in P_{L}} \sigma_{v}^{2} \sum_{p \in P_{L}^{c}} N^{\kappa} \widehat{V}\left(p / N^{1-\kappa}\right) \eta_{p} & +\frac{1}{N} \sum_{v \in P_{L}} \sigma_{v}^{2} \sum_{r \in P_{H}} N^{\kappa} \widehat{V}\left(r / N^{1-\kappa}\right)\left(\eta_{r}+\eta_{r+v}\right) \\
& \leq \frac{1}{N} \sum_{v \in P_{L}} \sigma_{v}^{2} \sum_{r \in P_{L}^{c}} N^{\kappa} \widehat{V}\left(r / N^{1-\kappa}\right) \eta_{r+v}+C N^{5 \kappa / 2-\varepsilon}
\end{aligned}
$$

Inserting (3.9), (3.10), (3.11) and (3.13) into (3.8), we obtain

$$
\begin{aligned}
E_{N}^{\Psi} \leq & \frac{N^{1+\kappa}}{2} \widehat{V}(0)+\sum_{p \in P_{L}^{c}}\left[p^{2} \eta_{p}+N^{\kappa} \widehat{V}\left(p / N^{1-\kappa}\right)+\frac{1}{2 N} \sum_{r \in P_{L}^{c}} N^{\kappa} \widehat{V}\left((r-p) / N^{\kappa}\right) \eta_{r}\right] \eta_{p} \\
& +\sum_{v \in P_{L}}\left[v^{2} \sigma_{v}^{2}+\left(\sigma_{v}^{2}+\gamma_{v} \sigma_{v}\right)\left(N^{\kappa} \widehat{V}\left(v / N^{1-\kappa}\right)+\frac{1}{N} \sum_{r \in P_{L}^{c}} N^{\kappa} \widehat{V}\left((r-v) / N^{1-\kappa}\right) \eta_{r}\right)\right] \\
& +C N^{5 \kappa / 2} \max \left\{N^{-\varepsilon}, N^{9 \kappa-5+6 \varepsilon}, N^{21 \kappa / 4-3+3 \varepsilon}\right\} .
\end{aligned}
$$

Let us now consider the first square bracket on the right-hand side of (3.14). Using the scattering equation (2.9) we obtain

$$
\begin{aligned}
\sum_{p \in P_{L}^{c}}\left[p^{2} \eta_{p}+N^{\kappa} \widehat{V}\left(p / N^{1-\kappa}\right)+\frac{1}{2 N} \sum_{r \in P_{L}^{c}} N^{\kappa} \widehat{V}\left((r-p) / N^{1-\kappa}\right) \eta_{r}\right] \eta_{p} \\
=\frac{1}{2} \sum_{p \in P_{L}^{c}} N^{\kappa} \widehat{V}\left(p / N^{1-\kappa}\right) \eta_{p}-\frac{1}{2 N} \sum_{\substack{p \in P_{L}^{c} \\
v \in P_{L}}} N^{\kappa} \widehat{V}\left((p-v) / N^{1-\kappa}\right) \eta_{v} \eta_{p}+\mathcal{E}_{2}
\end{aligned}
$$

with

$$
\mathcal{E}_{2}=N^{3-2 \kappa} \lambda_{\ell} \sum_{p \in P_{L}^{c}}\left(\widehat{\chi}_{\ell} * \widehat{f}_{N}, \ell\right)_{p} \eta_{p}-\frac{1}{2 N} \sum_{p \in P_{L}^{c}} N^{\kappa} \widehat{V}\left(p / N^{1-\kappa}\right) \eta_{p} \eta_{0}
$$

Using $N^{3-3 \kappa} \lambda_{\ell} \leq C$ (Lemma 2.1), $\left\|\widehat{\chi}_{\ell} * \widehat{f}_{N, \ell}\right\|=\left\|\chi \ell f_{N, \ell}\right\| \leq C$ and $\left\|\eta_{L^{c}}\right\|^{2} \leq C N^{3 \kappa / 2-\varepsilon}$ (Lemma 2.4) in the first term, (2.10) and (3.12) in the second term, we find $\mathcal{E}_{2} \leq C N^{5 \kappa / 2-\varepsilon}$ (using that $3 \kappa-2+4 \varepsilon<0$ and $\kappa>1 / 2)$. 
As for the second square bracket on the right-hand side of (3.14), we write

$$
\begin{aligned}
\sum_{v \in P_{L}}\left(\sigma_{v}^{2}+\gamma_{v} \sigma_{v}\right)\left(N^{\kappa} \widehat{V}\left(v / N^{1-\kappa}\right)\right. & \left.+\frac{1}{N} \sum_{r \in P_{L}^{c}} N^{\kappa} \widehat{V}\left((r-v) / N^{1-\kappa}\right) \eta_{r}\right) \\
& =\sum_{v \in P_{L}}\left(\sigma_{v}^{2}+\gamma_{v} \sigma_{v}\right) N^{\kappa}\left(\widehat{V}\left(\cdot / N^{1-\kappa}\right) * \widehat{f}_{N, \ell}\right)_{v}+\mathcal{E}_{3}
\end{aligned}
$$

with

$$
\mathcal{E}_{3}=-\frac{1}{N_{v}} \sum_{r \in P_{L}} N^{\kappa} \widehat{V}\left((r-v) / N^{1-\kappa}\right)\left(\sigma_{v}^{2}+\gamma_{v} \sigma_{v}\right) \eta_{r}-\frac{\eta_{0}}{N} \sum_{v \in P_{L}} N^{\kappa} \widehat{V}_{N}\left(v / N^{1-\kappa}\right)\left(\sigma_{v}^{2}+\gamma_{v} \sigma_{v}\right) .
$$

With (2.8), Lemma 2.4, $\left|\eta_{0}\right| \leq C N^{\kappa}$ and the assumption $3 \kappa-2+5 \varepsilon<0$, we obtain $\mathcal{E}_{3} \leq N^{5 \kappa / 2-\varepsilon}$.

Inserting (3.15) and (3.16) in (3.14) and completing sums over $p$ on the right-hand side of (3.15), we arrive at

$$
\begin{aligned}
E_{N}^{\Psi} \leq & \frac{N}{2}\left(N^{\kappa} \widehat{V}_{N}\left(\cdot / N^{1-\kappa}\right) * \widehat{f}_{N}, \ell\right)_{0} \\
& +\sum_{v \in P_{L}}\left[v^{2} \sigma_{v}^{2}+\left(\sigma_{v}^{2}+\gamma_{v} \sigma_{v}\right)\left(N^{\kappa} \widehat{V}\left(\cdot / N^{1-\kappa}\right) * \widehat{f}_{N, \ell}\right)_{v}-\frac{1}{2} N^{\kappa}\left(\widehat{V}\left(\cdot / N^{1-\kappa}\right) * \widehat{f}_{N, \ell}\right)_{v} \eta_{v}\right] \\
& +C N^{5 \kappa / 2} \max \left\{N^{-\varepsilon}, N^{9 \kappa-5+6 \varepsilon}, N^{21 \kappa / 4-3+3 \varepsilon}\right\} .
\end{aligned}
$$

Let us now introduce the notation $\hat{g}_{p}=\left(N^{\kappa} \widehat{V}\left(\cdot / N^{1-\kappa}\right) * \widehat{f}_{N, \ell}\right)_{p}$. Notice that

$$
\left|\widehat{g}_{0}-8 \pi \mathfrak{a} N^{\kappa}\right| \leq C N^{2 \kappa-1}, \quad\left|\widehat{g}_{p}-\widehat{g}_{0}\right| \leq C|p| N^{2 \kappa-1} .
$$

With the expression (2.28), we obtain

$$
\begin{aligned}
\sum_{v \in P_{L}}\left[v^{2} \sigma_{v}^{2}+\left(\sigma_{v}^{2}+\gamma_{v} \sigma_{v}\right) \widehat{g}_{v}\right] & =\frac{1}{2} \sum_{v \in P_{L}}\left[-v^{2}-\widehat{g}_{v}+\frac{v^{4}+v^{2}\left(8 \pi \mathfrak{a} N^{\kappa}+\widehat{g}_{v}\right)}{\sqrt{v^{4}+16 \pi \mathfrak{a} N^{\kappa} v^{2}}}\right] \\
& =\frac{1}{2} \sum_{v \in P_{L}}\left[\sqrt{v^{4}+16 \pi \mathfrak{a} N^{\kappa} v^{2}}-v^{2}-8 \pi \mathfrak{a} N^{\kappa}\right]+\mathcal{E}_{4}
\end{aligned}
$$

where, with (3.18) and $3 \kappa-2+4 \varepsilon<0$, we find

$$
\begin{aligned}
\mathcal{E}_{4} & =\frac{1}{2} \sum_{v \in P_{L}}\left[8 \pi \mathfrak{a} N^{\kappa}-\widehat{g}_{v}+\frac{\widehat{g}_{v}-8 \pi \mathfrak{a} N^{\kappa}}{\sqrt{1+16 \pi \mathfrak{a} N^{\kappa} / v^{2}}}\right] \\
& \leq C \sum_{v \in P_{L}}\left|8 \pi \mathfrak{a} N^{\kappa}-\widehat{g}_{v}\right| \frac{\left|\sqrt{1+16 \pi \mathfrak{a} N^{\kappa} / v^{2}}-1\right|}{\sqrt{1+16 \pi \mathfrak{a} N^{\kappa} / v^{2}}} \\
& \leq C N^{2 \kappa-1}\left[\sum_{|v|<N^{\kappa / 2}}(1+|v|)+\sum_{v \in P_{L}:|v|>N^{\kappa / 2}} \frac{N^{\kappa / 2}}{|v|^{2}}\right] \leq C N^{5 \kappa / 2-\varepsilon} .
\end{aligned}
$$

Moreover, from (3.17) and with the scattering equation (2.9), we obtain

$$
-\frac{N^{\kappa}}{2} \sum_{v \in P_{L}} \widehat{g}_{v} \eta_{v} \leq \sum_{v \in P_{L}} \frac{\left(8 \pi \mathfrak{a} N^{\kappa}\right)^{2}}{4 v^{2}}+C N^{5 \kappa / 2-\varepsilon}
$$


Thus,

$$
\begin{aligned}
E_{N}^{\Psi} \leq & 4 \pi \mathfrak{a} N^{1+\kappa}+\frac{1}{2} \sum_{v \in P_{L}}\left[\sqrt{v^{4}+16 \pi \mathfrak{a} N^{\kappa} v^{2}}-v^{2}-8 \pi \mathfrak{a} N^{\kappa}+\frac{\left(8 \pi \mathfrak{a} N^{\kappa}\right)^{2}}{2 v^{2}}\right] \\
& +C N^{5 \kappa / 2} \max \left\{N^{-\varepsilon}, N^{9 \kappa-5+6 \varepsilon}, N^{21 \kappa / 4-3+3 \varepsilon}\right\} .
\end{aligned}
$$

With

$$
\left|\sqrt{v^{4}+16 \pi \mathfrak{a} N^{\kappa} v^{2}}-v^{2}-8 \pi \mathfrak{a} N^{\kappa}+\frac{\left(8 \pi \mathfrak{a} N^{\kappa}\right)^{2}}{2 v^{2}}\right| \leq C \frac{N^{2 \kappa}}{|v|^{4}}
$$

we can replace, up to an error of order $N^{5 \kappa / 2-\varepsilon}$, the sum over $P_{L}$ with a sum over all $\Lambda_{+}^{*}$. With the rescaling $v \rightarrow N^{\kappa / 2} v$, we arrive at

$$
\begin{aligned}
E_{N}^{\Psi} \leq & 4 \pi \mathfrak{a} N^{1+\kappa}+\frac{N^{\kappa}}{2} \sum_{v \in 2 \pi N^{-\kappa / 2} \mathbb{Z}^{3}}\left[\sqrt{v^{4}+16 \pi \mathfrak{a} v^{2}}-v^{2}-8 \pi \mathfrak{a}+\frac{(8 \pi \mathfrak{a})^{2}}{2 v^{2}}\right] \\
& +C N^{5 \kappa / 2} \max \left\{N^{-\varepsilon}, N^{9 \kappa-5+6 \varepsilon}, N^{21 \kappa / 4-3+3 \varepsilon}\right\} .
\end{aligned}
$$

Recognising that (3.19) defines a Riemann sum and explicitly computing

$$
\frac{1}{2(2 \pi)^{3}} \int d v\left[\sqrt{v^{4}+16 \pi \mathfrak{a} v^{2}}-v^{2}-8 \pi \mathfrak{a}+\frac{(8 \pi \mathfrak{a})^{2}}{2 v^{2}}\right]=4 \pi \mathfrak{a} \cdot \frac{128}{15 \sqrt{\pi}} \mathfrak{a}^{3 / 2}
$$

we conclude that

$$
\begin{aligned}
E_{N}^{\Psi} \leq & 4 \pi \mathfrak{a} N^{1+\kappa} \cdot\left[1+\frac{128}{15 \sqrt{\pi}}\left(\mathfrak{a}^{3} N^{3 \kappa-2}\right)^{1 / 2}\right] \\
& +C N^{5 \kappa / 2} \max \left\{N^{-\varepsilon}, N^{9 \kappa-5+6 \varepsilon}, N^{21 \kappa / 4-3+3 \varepsilon}\right\} .
\end{aligned}
$$

To compare the Riemann sum in (3.19) with the integral, we first removed contributions arising from $|v| \leq N^{-\varepsilon}$ using that $|F(v)| \leq C / v^{2}$, for small $v$, with the definition $F(v)=\sqrt{v^{4}+16 \pi \mathfrak{a} v^{2}}-v^{2}-8 \pi \mathfrak{a}+$ $(8 \pi \mathfrak{a})^{2} / 2 v^{2}$. For $|v|>N^{-\varepsilon}$, we use that $|\nabla F(v)| \leq C|v|^{-3}\left(1+v^{2}\right)^{-1}$ to compare the value of $F(q)$ with $F(v)$, for all $q$ in the cube of size $2 \pi N^{-\kappa / 2}$ centred at $v$.

\section{Bogoliubov transformation}

In this section, we show Proposition 3.1. From the definition (3.1) and from (3.3), we obtain (since $T_{v}$ does not act on the zero momentum mode and since $a_{0} \xi_{v}=0$ )

$$
\frac{\left\langle\xi_{v}, \mathcal{G}_{N} \xi_{v}\right\rangle}{\left\|\xi_{v}\right\|^{2}}=\frac{N_{0}^{2}}{2} N^{\kappa-1} \widehat{V}(0)+\sum_{j=2}^{4} \frac{\left\langle\xi_{v}, \mathcal{G}_{N}^{(j)} \xi_{v}\right\rangle}{\left\|\xi_{v}\right\|^{2}}
$$

with $\mathcal{G}_{N}^{(j)}=T_{v}^{*} \mathcal{L}_{N}^{(j)} T_{v}$, for $j=2,3,4$.

We start from the contribution of $\mathcal{G}_{N}^{(2)}$. We write $\mathcal{L}_{N}^{(2)}=\mathcal{K}+\mathcal{L}_{N}^{(2, V)}$ with

$$
\mathcal{L}_{N}^{(2, V)}=\frac{N_{0}}{N} \sum_{p \in \Lambda^{*}} N^{\kappa}\left(\hat{V}\left(p / N^{1-\kappa}\right)+\hat{V}(0)\right) a_{p}^{*} a_{p}+\frac{N_{0}}{2 N} \sum_{p \in \Lambda^{*}} N^{\kappa} \hat{V}\left(p / N^{1-\kappa}\right)\left(a_{p}^{*} a_{-p}^{*}+\text { h.c. }\right) .
$$


Using (2.14) we get

$$
T_{v}^{*} \mathcal{K} T_{v}-\left[\mathcal{K}+\sum_{p \in \Lambda_{+}^{*}} p^{2} \sigma_{p}^{2}\right]=2 \sum_{p \in \Lambda_{+}^{*}} p^{2} \sigma_{p}^{2} a_{p}^{*} a_{p}+\sum_{p \in \Lambda_{+}^{*}} p^{2}\left[\gamma_{p} \sigma_{p}\left(a_{p}^{*} a_{-p}^{*}+\text { h.c. }\right)\right]:=E_{1}+E_{2} .
$$

From (2.15), $\left\langle\xi_{v}, \mathrm{E}_{2} \xi_{v}\right\rangle=0$ ( $\xi_{v}$ is a superposition of states with $3 m$ particles, for $m \in \mathbb{N}$ ). To bound the expectation of $E_{1}$ on $\xi_{v}$ we notice that $\left\langle\xi_{v}, a_{p}^{*} a_{p} \xi_{v}\right\rangle=0$ if $p \in \Lambda_{+}^{*} \backslash\left(P_{S} \cup P_{H}\right)$. Moreover, proceeding as in (2.31), we have

$$
\sup _{p \in P_{S}}\left(p^{2} \sigma_{p}^{2}\right) \leq \sup _{p \in P_{S}:|p| \leq N^{\kappa / 2}} N^{\kappa / 2}|p|+\sup _{p \in P_{S}:|p|>N^{\kappa / 2}} \frac{N^{2 \kappa}}{p^{2}} \leq C N^{\kappa}
$$

while

$$
\sup _{p \in P_{H}}\left(p^{2} \sigma_{p}^{2}\right) \leq \sup _{|p| \geq N^{1-\kappa-\varepsilon}} \frac{N^{2 \kappa}}{p^{2}} \leq C N^{-2+4 \kappa+2 \varepsilon} \leq C N^{\kappa}
$$

because, by assumption, $3 \kappa-2+2 \varepsilon<0$. Hence,

$$
\left|\left\langle\xi_{v}, E_{1} \xi_{v}\right\rangle\right| \leq C N^{\kappa}\left\|\mathcal{N}^{1 / 2} \xi_{v}\right\|^{2} .
$$

We now consider the contribution from $\mathcal{L}_{N}^{(2, V)}$. Using again $\left\langle\xi_{v}, a_{p}^{*} a_{-p}^{*} \xi_{v}\right\rangle=0$ for all $p \in \Lambda_{+}^{*}$ and $\left\langle\xi_{v}, a_{p}^{*} a_{p} \xi_{v}\right\rangle=0$ for all $p \in \Lambda_{+}^{*} \backslash\left(P_{S} \cup P_{H}\right)$, a straightforward computation shows that

$$
\begin{aligned}
\frac{\left\langle\xi_{v}, T_{v}^{*} \mathcal{L}_{N}^{(2, V)} T_{\nu} \xi_{v}\right\rangle}{\left\|\xi_{v}\right\|^{2}} & \\
= & \frac{N_{0}}{N} \sum_{p \in \Lambda_{+}^{*}} N^{\kappa} \widehat{V}\left(p / N^{1-\kappa}\right) \gamma_{p} \sigma_{p}+\frac{N_{0}}{N} \sum_{p \in \Lambda_{+}^{*}} N^{\kappa}\left(\widehat{V}(0)+\widehat{V}\left(p / N^{1-\kappa}\right)\right) \sigma_{p}^{2} \\
& +\frac{N_{0}}{N} \sum_{p \in P_{S} \cup P_{H}} N^{\kappa}\left[\widehat{V}\left(p / N^{\kappa-1}\right)\left(\gamma_{p}+\sigma_{p}\right)^{2}+\widehat{V}(0)\left(\gamma_{p}^{2}+\sigma_{p}^{2}\right)\right] \frac{\left\langle\xi_{v}, a_{p}^{*} a_{p} \xi_{v}\right\rangle}{\left\|\xi_{v}\right\|^{2}}
\end{aligned}
$$

With the bounds $\left\|\gamma_{S}\right\|_{\infty}^{2},\left\|\sigma_{S}\right\|_{\infty}^{2},\left\|\sigma_{H}\right\|_{\infty}^{2},\left\|\gamma_{H}\right\|_{\infty}^{2} \leq C N^{\varepsilon}$ from Lemma 2.4, with (4.1) and with the estimate $\left\langle\xi_{v}, \mathcal{N} \xi_{v}\right\rangle \leq C N^{9 \kappa / 2-2+\varepsilon}\left\|\xi_{v}\right\|^{2}$ from Proposition 2.2, we conclude that

$$
\begin{aligned}
\frac{\left\langle\xi_{v}, \mathcal{G}_{N}^{(2)} \xi_{v}\right\rangle}{\left\|\xi_{v}\right\|^{2}} \leq & \frac{\left\langle\xi_{v}, \mathcal{K} \xi_{v}\right\rangle}{\left\|\xi_{v}\right\|^{2}}+\sum_{p \in \Lambda_{+}^{*}} p^{2} \sigma_{p}^{2}+\frac{N_{0}}{N} \sum_{p \in \Lambda_{+}^{*}} N^{\kappa} \widehat{V}\left(p / N^{1-\kappa}\right) \gamma_{p} \sigma_{p} \\
& +\frac{N_{0}}{N} \sum_{p \in \Lambda_{+}^{*}} N^{\kappa}\left(\widehat{V}(0)+\widehat{V}\left(p / N^{1-\kappa}\right)\right) \sigma_{p}^{2}+C N^{5 \kappa / 2-\varepsilon}
\end{aligned}
$$

using again the condition $3 \kappa-2+4 \varepsilon<0$.

Next, we study the contribution of $\mathcal{G}_{N}^{(3)}=T_{v}^{*} \mathcal{L}_{N}^{(3)} T_{\nu}$, with $\mathcal{L}_{N}^{(3)}$ as in (3.3). Recall the operator $\mathcal{C}_{N}$, defined in (3.5). Taking into account the fact that $\xi_{v}$ is a superposition of vectors with $2 m$ particles with momenta in $P_{H}$ and $m$ particles with momenta in $P_{S}$, for $m \in \mathbb{N}$, we obtain that

$$
\left\langle\xi_{v}, \mathcal{G}_{N}^{(3)} \xi_{v}\right\rangle=\left\langle\xi_{v}, \mathcal{C}_{N} \xi_{v}\right\rangle+\sum_{j=1}^{3}\left[\left\langle\xi_{v}, \mathrm{~F}_{j} \xi_{v}\right\rangle+\text { h.c. }\right]
$$


with

$$
\begin{aligned}
& \mathrm{F}_{1}=\frac{\sqrt{N_{0}}}{N} \sum_{p, r \in P_{H}: p+r \in P_{S}} N^{\kappa} \widehat{V}\left(r / N^{1-\kappa}\right) \gamma_{p+r} \sigma_{p} \sigma_{r} a_{p+r}^{*} a_{-p}^{*} a_{-r}^{*} \\
& \mathrm{~F}_{2}=\frac{\sqrt{N_{0}}}{N} \sum_{p \in P_{H}, r \in P_{S}: p+r \in P_{H}} N^{\kappa}\left[\widehat{V}\left(r / N^{1-\kappa}\right)+\widehat{V}\left(p / N^{1-\kappa}\right)\right] \gamma_{p+r} \sigma_{p} \sigma_{r} a_{p+r}^{*} a_{-p}^{*} a_{-r}^{*} \\
& \mathrm{~F}_{3}=\frac{\sqrt{N_{0}}}{N} \sum_{p \in P_{H}, r \in P_{S}: p+r \in P_{H}} N^{\kappa}\left[\widehat{V}\left(r / N^{1-\kappa}\right)+\widehat{V}\left(p / N^{1-\kappa}\right)\right] \sigma_{p+r} \gamma_{p} \gamma_{r} a_{-p-r} a_{p} a_{r}
\end{aligned}
$$

Using $\left\|a_{-r}^{*}(\mathcal{N}+1)^{1 / 2} \xi_{v}\right\| \leq\left\|a_{-r}(\mathcal{N}+1)^{1 / 2} \xi_{v}\right\|+\left\|(\mathcal{N}+1)^{1 / 2} \xi_{v}\right\|$, we can bound

$$
\begin{aligned}
&\left\langle\xi_{v}, \mathrm{~F}_{1} \xi_{v}\right\rangle \leq C N^{K-1 / 2}\left\|\gamma_{S}\right\|_{\infty} \sum_{p, r \in P_{H}}\left|\sigma_{r}\left\|\sigma_{p} \mid\right\| a_{p+r} a_{-p}(\mathcal{N}+1)^{-1 / 2} \xi_{v} \|\right. \\
& \times\left[\left\|a_{-r}(\mathcal{N}+1)^{1 / 2} \xi_{v}\right\|+\left\|(\mathcal{N}+1)^{1 / 2} \xi_{v}\right\|\right] \\
& \leq C N^{K-1 / 2}\left\|\gamma_{S}\right\|_{\infty}\left\|\sigma_{H}\right\|_{\infty}\left\|\sigma_{H}\right\|\left\|(\mathcal{N}+1)^{1 / 2} \xi_{v}\right\|\left\|(\mathcal{N}+1) \xi_{v}\right\| \\
&+C N^{K-1 / 2}\left\|\gamma_{S}\right\|_{\infty}\left\|\sigma_{H}\right\|^{2}\left\|(\mathcal{N}+1)^{1 / 2} \xi_{v}\right\|^{2} .
\end{aligned}
$$

With Lemma 2.4 and Proposition 2.2, we obtain

$$
\frac{\left\langle\xi_{v}, \mathrm{~F}_{1} \xi_{\nu}\right\rangle}{\left\|\xi_{v}\right\|^{2}} \leq C N^{37 \kappa / 4-4+5 \varepsilon / 2}+C N^{17 \kappa / 2-7 / 2+5 \varepsilon / 2} \leq C N^{5 \kappa / 2} \cdot N^{21 \kappa / 4-7 / 2+3 \varepsilon / 2}
$$

from the assumption that $3 \kappa-2+4 \varepsilon<0$. Similarly, we find

$$
\begin{aligned}
\left\langle\xi_{v}, \mathrm{~F}_{2} \xi_{v}\right\rangle \leq & C N^{\kappa-1 / 2}\left\|\gamma_{H}\right\|_{\infty}\left\|\sigma_{S}\right\|_{\infty}\left\|\sigma_{H}\right\|\left\|(\mathcal{N}+1)^{1 / 2} \xi_{v}\right\|\left\|(\mathcal{N}+1) \xi_{v}\right\| \\
& +C N^{\kappa-1 / 2}\left\|\gamma_{H}\right\|_{\infty}\left\|\sigma_{S}\right\|\left\|\sigma_{H}\right\|\left\|(\mathcal{N}+1)^{1 / 2} \xi_{v}\right\|^{2} \\
\leq & C\left[N^{37 \kappa / 4-4+3 \varepsilon}+N^{31 \kappa / 4-3+3 \varepsilon / 2}\right]\left\|\xi_{v}\right\|^{2} \leq C N^{5 \kappa / 2} \cdot N^{21 \kappa / 4-3+3 \varepsilon / 2}\left\|\xi_{v}\right\|^{2}
\end{aligned}
$$

and also

$$
\begin{aligned}
\left\langle\xi_{v}, \mathrm{~F}_{3} \xi_{v}\right\rangle \leq & C N^{\kappa-1 / 2}\left\|\gamma_{H}\right\|_{\infty}\left\|\gamma_{S}\right\|_{\infty}\left\|\sigma_{H}\right\|\left\|(\mathcal{N}+1)^{1 / 2} \xi_{v}\right\|\left\|(\mathcal{N}+1) \xi_{v}\right\| \\
& +C N^{\kappa-1 / 2}\left\|\gamma_{H}\right\|_{\infty}\left\|\gamma_{S}\right\|\left\|\sigma_{H}\right\|\left\|(\mathcal{N}+1)^{1 / 2} \xi_{v}\right\|^{2} \\
\leq & C N^{5 \kappa / 2} \cdot N^{21 \kappa / 4-3+3 \varepsilon}\left\|\xi_{v}\right\|^{2}
\end{aligned}
$$

Summarising, we have

$$
\frac{\left\langle\xi_{v}, \mathcal{G}_{N}^{(3)} \xi_{v}\right\rangle}{\left\|\xi_{v}\right\|^{2}} \leq \frac{\left\langle\xi_{v}, \mathcal{C}_{N} \xi_{v}\right\rangle}{\left\|\xi_{v}\right\|^{2}}+C N^{5 \kappa / 2} \cdot N^{21 \kappa / 4-3+3 \varepsilon}
$$


Finally, let us consider $\mathcal{G}_{N}^{(4)}=T_{v}^{*} \mathcal{L}_{N}^{(4)} T_{v}$. We decompose $\left\langle\xi_{v}, \mathcal{G}_{N}^{(4)} \xi_{v}\right\rangle=\sum_{j=1}^{3}\left\langle\xi_{v}, \mathrm{G}_{j} \xi_{v}\right\rangle$ with

$$
\begin{aligned}
& \mathrm{G}_{1}= \frac{1}{2 N} \sum_{\substack{r \in \Lambda^{*}, p, q \in \Lambda_{+}^{*} \\
-r \neq q, p}} N^{\kappa} \widehat{V}\left(r / N^{1-\kappa}\right) \gamma_{p} \gamma_{q} \gamma_{p+r} \gamma_{q+r} a_{p+r}^{*} a_{q}^{*} a_{p} a_{q+r} \\
& \mathrm{G}_{2}=\frac{1}{2 N} \sum_{\substack{r \in \Lambda^{*}, p, q \in \Lambda_{+}^{*} \\
r \neq q,-p}} N^{\kappa} \widehat{V}\left(r / N^{1-\kappa}\right)\left(\gamma_{p+r} \sigma_{q} a_{p+r}^{*} a_{-q}+\sigma_{p+r} \gamma_{q} a_{-p-r} a_{q}^{*}\right) \\
& \mathrm{G}_{3}=\frac{1}{2 N} \sum_{\substack{r \in \Lambda^{*}, p, q \in \Lambda_{+}^{*} \\
r \neq q,-p}} N^{\kappa} \widehat{V}\left(r / N^{1-\kappa}\right) \sigma_{p} \sigma_{q} \sigma_{p+r} \sigma_{q+r} a_{p+r} a_{q} a_{p}^{*} a_{q+r}^{*} .
\end{aligned}
$$

To estimate contributions from $\mathrm{G}_{3}$, we arrange terms in normal order. We find

$$
\begin{aligned}
\mathrm{G}_{3}= & \frac{1}{2 N} \sum_{\substack{r \in \Lambda^{*}, p, q \in \Lambda_{+}^{*} \\
-r \neq q, p}} N^{\kappa} \widehat{V}\left(r / N^{1-\kappa}\right) \sigma_{p} \sigma_{q} \sigma_{p+r} \sigma_{q+r} a_{p}^{*} a_{q+r}^{*} a_{p+r} a_{q} \\
& +\frac{1}{2 N} \sum_{\substack{r \in \Lambda^{*}, p \in \Lambda_{+}^{*} \\
p \neq-r}} N^{\kappa} \widehat{V}\left(r / N^{1-\kappa}\right) \sigma_{p}^{2} \sigma_{p+r}^{2}\left(a_{p}^{*} a_{p}+a_{p+r}^{*} a_{p+r}\right) \\
& +\frac{1}{N} \sum_{p, q \in \Lambda_{+}^{*}} N^{\kappa} \widehat{V}(0) \sigma_{p}^{2} \sigma_{q}^{2} a_{p}^{*} a_{p} \\
& +\frac{1}{2 N} \sum_{\substack{r \in \Lambda^{*}, p \in \Lambda_{+}^{*} \\
p \neq-r}} N^{\kappa} \widehat{V}\left(r / N^{1-\kappa}\right) \sigma_{p}^{2} \sigma_{p+r}^{2}+\frac{1}{2 N} \sum_{p, q \in \Lambda_{+}^{*}} N^{\kappa} \widehat{V}(0) \sigma_{p}^{2} \sigma_{q}^{2} .
\end{aligned}
$$

Since $a_{p} \xi_{v}=0$ if $p \in \Lambda_{+}^{*} \backslash\left(P_{S} \cup P_{H}\right)$ and $\left\|\sigma_{H}\right\|_{\infty} \leq\left\|\sigma_{S}\right\|_{\infty}$, we find, by Cauchy-Schwarz,

$$
\begin{aligned}
\left\langle\xi_{v}, \mathrm{G}_{3} \xi_{v}\right\rangle & \leq C N^{\kappa-1}\left[\left\|\sigma_{S}\right\|_{\infty}^{2}\|\sigma\|^{2}\left\|(\mathcal{N}+1) \xi_{v}\right\|^{2}+\|\sigma\|^{4}\left\|\xi_{v}\right\|^{2}\right] \\
& \leq C N^{5 \kappa / 2} \cdot \max \left\{N^{-\varepsilon}, N^{9 \kappa-5+3 \varepsilon}\right\}\left\|\xi_{v}\right\|^{2}
\end{aligned}
$$

using Proposition 2.2 and $3 \kappa-2+4 \varepsilon<0$. We proceed similarly for $\mathrm{G}_{2}$. Through normal ordering, we get

$$
\begin{aligned}
\mathrm{G}_{2}= & \frac{1}{N} \sum_{\substack{r \in \Lambda^{*}, p, q \in \Lambda_{+}^{*} \\
-r \neq q, p}} N^{\kappa} \widehat{V}\left(r / N^{1-\kappa}\right) \gamma_{p} \gamma_{p+r} \sigma_{q} \sigma_{q+r} a_{p+r}^{*} a_{-q-r}^{*} a_{p} a_{-q} \\
& +\frac{1}{N} \sum_{\substack{r \in \Lambda^{*}, p, q \in \Lambda_{+}^{*} \\
-r \neq q, p}} N^{\kappa} \widehat{V}\left(r / N^{1-\kappa}\right) \gamma_{p+r} \gamma_{q+r} \sigma_{p} \sigma_{q} a_{p+r}^{*} a_{-p}^{*} a_{-q} a_{q+r} \\
& +\frac{1}{N} \sum_{p, q \in \Lambda_{+}^{*}} N^{\kappa} \widehat{V}(0) \gamma_{p}^{2} \sigma_{q}^{2} a_{p}^{*} a_{p}+\frac{1}{N} \sum_{r \in \Lambda^{*}, p \in \Lambda_{+}^{*}} N^{\kappa} \widehat{V}\left(r / N^{1-\kappa}\right) \gamma_{p}^{2} \sigma_{p+r}^{2} a_{p}^{*} a_{p} \\
& +\frac{2}{N} \sum_{r \in \Lambda^{*}, p \in \Lambda_{+}^{*}} N^{\kappa} \widehat{V}\left(r / N^{1-\kappa}\right) \gamma_{p} \sigma_{p} \gamma_{p+r} \sigma_{p+r} a_{p}^{*} a_{p} \\
& +\frac{1}{2 N} \sum_{p, r \in \Lambda_{+}^{*}} N^{\kappa} \widehat{V}\left(r / N^{1-\kappa}\right) \gamma_{p} \gamma_{p+r} \sigma_{p} \sigma_{p+r} .
\end{aligned}
$$


Keeping the last contribution intact and estimating the term on the fourth line distinguishing the two cases $(p+r) \in P_{S}$ and $(p+r) \in P_{H}$, we arrive at

$$
\begin{aligned}
\left\langle\xi_{v}, \mathrm{G}_{2} \xi_{v}\right\rangle \leq & \frac{1}{2 N} \sum_{p, r \in \Lambda_{+}^{*}} N^{\kappa} \widehat{V}\left(r / N^{1-\kappa}\right) \gamma_{p} \gamma_{p+r} \sigma_{p} \sigma_{p+r}\left\|\xi_{v}\right\|^{2} \\
& +C N^{\kappa-1}\left\|\gamma_{S}\right\|_{\infty}^{2}\|\sigma\|^{2}\left\|(\mathcal{N}+1) \xi_{v}\right\|^{2} \\
& +C N^{\kappa-1}\left\|\gamma_{S \cup H}\right\|_{\infty}\left\|\sigma_{S \cup H}\right\|_{\infty} \\
& \times\left[\left\|\gamma_{S} \sigma_{S}\right\|_{1}+\left\|\gamma_{H}\right\|_{\infty} \sup _{p} \sum_{r \in \Lambda^{*}} \widehat{V}\left(r / N^{1-\kappa}\right)\left|\eta_{p+r}\right|\right]\left\|\mathcal{N}^{1 / 2} \xi_{v}\right\|^{2} .
\end{aligned}
$$

With the bounds in Lemma 2.4 and in Proposition 2.2 and with (3.12), we conclude that

$$
\left\langle\xi_{\nu}, \mathrm{G}_{2} \xi_{v}\right\rangle \leq \frac{1}{2 N} \sum_{p, r \in \Lambda_{+}^{*}} N^{\kappa} \widehat{V}\left(r / N^{1-\kappa}\right) \gamma_{p} \gamma_{p+r} \sigma_{p} \sigma_{p+r}\left\|\xi_{\nu}\right\|^{2}+C N^{5 \kappa / 2} \cdot N^{9 \kappa-5+3 \varepsilon}\left\|\xi_{v}\right\|^{2}
$$

Finally, we consider $\mathrm{G}_{1}$. Recalling that $a_{p} \xi_{v}=0$ if $p \in \Lambda_{+}^{*} \backslash\left(P_{S} \cup P_{H}\right)$ and observing that $\left\langle\xi_{v}, a_{p+r}^{*} a_{q}^{*} a_{p} a_{q+r} \xi_{v}\right\rangle \neq 0$ only if the operator $a_{p+r}^{*} a_{q}^{*} a_{p} a_{q+r}$ preserves the number of particles in $P_{S}$ and in $P_{H}$, we arrive at

$$
\begin{aligned}
\left\langle\xi_{v}, \mathrm{G}_{1} \xi_{v}\right\rangle \leq & \frac{1}{2 N} \sum_{\substack{r \in \Lambda^{*}, p, q \in P_{H} \\
p+r, q+r \in P_{H}}} N^{\kappa} \widehat{V}\left(r / N^{1-\kappa}\right) \gamma_{p} \gamma_{q} \gamma_{p+r} \gamma_{q-r}\left\langle\xi_{v}, a_{p+r}^{*} a_{q}^{*} a_{p} a_{q+r} \xi_{v}\right\rangle \\
& +C N^{\kappa-1}\left\|\gamma_{S \cup H}\right\|_{\infty}^{2}\left\|\gamma_{S}\right\|^{2}\|(\mathcal{N}+1) \xi\|^{2}
\end{aligned}
$$

With $\left|\gamma_{p} \gamma_{q} \gamma_{p+r} \gamma_{q+r}-1\right| \leq C\left\|\eta_{H}\right\|_{\infty}^{2}$ for all $p, q \in P_{H}$, with $(p+r),(q+r) \in P_{H}$ and using the estimate (see the proof of (3.12))

$$
\sup _{p \in \Lambda^{*}} \sum_{r \in \Lambda_{+}^{*}: r \neq p} \frac{N^{\kappa}\left|\widehat{V}\left(r / N^{1-\kappa}\right)\right|}{|p-r|^{2}} \leq C N
$$

we conclude that

$$
\left\langle\xi_{v}, \mathrm{G}_{1} \xi_{v}\right\rangle \leq\left\langle\xi_{v}, \mathcal{V}_{N}^{(H)} \xi_{v}\right\rangle+C\left\|\eta_{H}\right\|_{\infty}^{2}\left\|\mathcal{N}^{1 / 2} \mathcal{K}^{1 / 2} \xi_{\nu}\right\|^{2}+C N^{\kappa-1}\left\|\gamma_{S \cup H}\right\|_{\infty}^{2}\left\|\gamma_{S}\right\|^{2}\|\mathcal{N} \xi\|^{2}
$$

with $\mathcal{V}_{N}^{(H)}$ defined as in (3.4). With Lemma 2.4 and Proposition 2.2, we find (using the assumption $3 \kappa-2+4 \varepsilon<0)$

$$
\left\langle\xi_{v}, \mathrm{G}_{1} \xi_{v}\right\rangle \leq\left\langle\xi_{v}, \mathcal{V}_{N}^{(H)} \xi_{v}\right\rangle+C N^{5 \kappa / 2} \cdot N^{9 \kappa-5+6 \varepsilon}\left\|\xi_{v}\right\|^{2}
$$

With (4.4) and (4.5), we have shown that

$$
\frac{\left\langle\xi_{v}, \mathcal{G}_{N}^{(4)} \xi_{v}\right\rangle}{\left\|\xi_{v}\right\|^{2}} \leq \frac{\left\langle\xi_{v}, \mathcal{V}_{N}^{(H)} \xi_{v}\right\rangle}{\left\|\xi_{v}\right\|^{2}}+C N^{5 \kappa / 2} \cdot \max \left\{N^{-\varepsilon}, N^{9 \kappa-5+6 \varepsilon}\right\}
$$

Combining the last bound with (4.2) and (4.3), we obtain

$$
\begin{aligned}
\frac{\left\langle\xi_{v}, \mathcal{G}_{N} \xi_{v}\right\rangle}{\left\|\xi_{v}\right\|^{2}} \leq & \widetilde{C}_{N}+\frac{\left\langle\xi_{v},\left(\mathcal{K}+\mathcal{V}_{N}^{(H)}+\mathcal{C}_{N}\right) \xi_{v}\right\rangle}{\left\|\xi_{v}\right\|^{2}} \\
& +C N^{5 \kappa / 2} \cdot \max \left\{N^{-\varepsilon}, N^{9 \kappa-5+6 \varepsilon}, N^{21 \kappa / 4-3+3 \varepsilon}\right\}
\end{aligned}
$$


where we defined

$$
\begin{aligned}
\widetilde{C}_{N}= & \frac{N_{0}^{2}}{2 N} N^{\kappa} \widehat{V}(0)+\sum_{p \in \Lambda_{+}^{*}} p^{2} \sigma_{p}^{2}+\frac{N_{0}}{N} \sum_{p \in \Lambda_{+}^{*}} N^{\kappa}\left(\widehat{V}\left(p / N^{1-\kappa}\right)+\widehat{V}(0)\right) \sigma_{p}^{2} \\
& +\frac{N_{0}}{N} \sum_{p \in \Lambda_{+}^{*}} N^{\kappa} \widehat{V}\left(p / N^{1-\kappa}\right) \sigma_{p} \gamma_{p}+\frac{1}{2 N} \sum_{\substack{p, r \in \Lambda_{+}^{*} \\
r \neq p}} N^{\kappa} \widehat{V}\left(r / N^{1-\kappa}\right) \sigma_{p} \sigma_{p+r} \gamma_{p} \gamma_{p+r} .
\end{aligned}
$$

Inserting $N_{0}=N-\left\|\sigma_{L}\right\|^{2}$ and recalling from Lemma 2.4 that $\left\|\sigma_{L}\right\|^{2} \leq C N^{3 \kappa / 2}$ and $\left\|\sigma_{L^{c}}\right\|^{2} \leq$ $C N^{3 \kappa / 2-\varepsilon}$, we obtain $\widetilde{C}_{N}=C_{\mathcal{G}_{N}}+\mathcal{O}\left(N^{5 \kappa / 2-\varepsilon}\right)$, with $C_{\mathcal{G}_{N}}$ as defined in (3.6) (with the assumption $3 \kappa-2+4 \varepsilon<0$ ). To handle the first term on the second line of (4.6), we used that $\left|\sigma_{p} \gamma_{p}-\eta_{p}\right| \leq C \eta_{p}^{3} \leq$ $C N^{3 \kappa} /|p|^{6}$, for $p \in P_{L}^{c}$. This completes the proof of Proposition 3.1.

\section{Cubic conjugation}

In this section we prove Proposition 2.2 and Proposition 3.2, as a consequence of the following lemma.

Lemma 5.1. Let $A_{v}$ be defined in (2.15) and $\mathcal{K}, \mathcal{V}_{N}^{(H)}$ and $\mathcal{C}_{N}$ be defined in (3.4) and (3.5), respectively. Then, for $\xi_{v}=e^{A_{v}} \Omega$,

$$
\begin{gathered}
\frac{\left\langle\xi_{v}, \mathcal{K} \xi_{v}\right\rangle}{\left\|\xi_{v}\right\|^{2}} \leq \frac{2}{N} \sum_{\substack{v \in P_{S}, r \in P_{H}: \\
r+v \in P_{H}}} r^{2} \eta_{r}\left(\eta_{r}+\eta_{r+v}\right) \sigma_{v}^{2}+\mathcal{E}, \\
\frac{\left\langle\xi_{v}, \mathcal{C}_{N} \xi_{v}\right\rangle}{\left\|\xi_{v}\right\|^{2}} \leq \frac{2}{N} \sum_{\substack{v \in P_{S}, r \in P_{H}: \\
r+v \in P_{H}}} N^{\kappa} \widehat{V}\left(r / N^{1-\kappa}\right)\left(\eta_{r}+\eta_{r+v}\right) \sigma_{v}^{2}+\mathcal{E}, \\
\frac{\left\langle\xi_{v}, \mathcal{V}_{N}^{(H)} \xi_{v}\right\rangle}{\left\|\xi_{v}\right\|^{2}} \leq \frac{1}{N^{2}} \sum_{\substack{v \in P_{S}, r \in P_{H}: \\
r+v \in P_{H}}}\left(N^{\kappa} \widehat{V}\left(\cdot / N^{1-\kappa}\right) * \eta\right)_{r}\left(\eta_{r}+\eta_{r+v}\right) \sigma_{v}^{2}+\mathcal{E},
\end{gathered}
$$

with

$$
\mathcal{E} \leq C N^{5 \kappa / 2} \cdot \max \left\{N^{-\varepsilon}, N^{12 \kappa-7+5 \varepsilon}\right\}
$$

for all $\kappa \in(1 / 2 ; 2 / 3), \varepsilon>0$ so small that $3 \kappa-2+4 \varepsilon<0$ and $N$ large enough.

With Lemma 5.1, we can immediately show Proposition 3.2.

Proof of Proposition 3.2. From Lemma 5.1 we have

$$
\begin{aligned}
& \frac{\left\langle\xi_{v},\left(\mathcal{K}+\mathcal{V}_{N}^{(H)}+\mathcal{C}_{N}\right) \xi_{v}\right\rangle}{\left\|\xi_{v}\right\|^{2}} \\
& \leq \frac{2}{N} \sum_{v \in P_{S}} \sigma_{v}^{2} \sum_{\substack{r \in P_{H}: \\
r+v \in P_{H}}}\left[r^{2} \eta_{r}+N^{\kappa} \widehat{V}\left(r / N^{1-\kappa}\right)+\frac{N^{\kappa}}{2 N}\left(\widehat{V}\left(\cdot / N^{1-\kappa}\right) * \eta\right)_{r}\right]\left(\eta_{r}+\eta_{r+v}\right)+\mathcal{E}
\end{aligned}
$$

with $\mathcal{E} \leq C N^{5 \kappa / 2} \cdot \max \left\{N^{-\varepsilon}, N^{12 \kappa-7+5 \varepsilon}\right\}$. With the scattering equation (2.9), we obtain

$$
\frac{\left\langle\xi_{v},\left(\mathcal{K}+\mathcal{V}_{N}^{(H)}+\mathcal{C}_{N}\right) \xi_{v}\right\rangle}{\left\|\xi_{v}\right\|^{2}} \leq \frac{1}{N} \sum_{v \in P_{S}} \sigma_{v}^{2} \sum_{\substack{r \in P_{H}: \\ r+v \in P_{H}}} N^{\kappa} \widehat{V}\left(r / N^{1-\kappa}\right)\left(\eta_{r}+\eta_{r+v}\right)+\mathcal{E}^{\prime}
$$


with

$$
\mathcal{E}^{\prime} \leq \frac{1}{N} \sum_{v \in P_{S}} \sigma_{v}^{2} \sum_{r \in P_{H}: r+v \in P_{H}} N^{3-2 \kappa} \lambda_{\ell}\left(\widehat{\chi}_{\ell} * \widehat{f}_{N}, \ell\right)_{r} \eta_{r}+\mathcal{E}
$$

Using $\left|N^{3-3 \kappa} \lambda_{\ell}\right| \leq C$ and $\left\|\widehat{\chi}_{\ell} * \widehat{f}_{N, \ell}\right\| \leq C$, we conclude

$$
\begin{aligned}
& \frac{\left\langle\xi_{v},\left(\mathcal{K}+\mathcal{V}_{N}^{(H)}+\mathcal{C}_{N}\right) \xi_{v}\right\rangle}{\left\|\xi_{v}\right\|^{2}} \\
& \quad \leq \frac{1}{N} \sum_{v \in P_{S}} \sigma_{v}^{2} \sum_{\substack{r \in P_{H}: \\
r+v \in P_{H}}} N^{\kappa} \widehat{V}\left(r / N^{1-\kappa}\right)\left(\eta_{r}+\eta_{r+v}\right)+C N^{5 \kappa / 2} \cdot \max \left\{N^{-\varepsilon}, N^{12 \kappa-7+5 \varepsilon}\right\} .
\end{aligned}
$$

Finally, with (3.12) and the expression (2.28) for $\sigma_{v}^{2}$, we can extend the sum over $v \in P_{S}$ to a sum over all $v \in P_{L}$, without changing the size of the error. This completes the proof of Proposition 3.2.

We still have to show Proposition 2.2 and Lemma 5.1.

\subsection{Expectation of the particle number and kinetic energy}

In this section we prove (5.1) and Proposition 2.2. We start by computing the expectation $\left\langle\xi_{\nu}, \mathcal{K} \xi_{\nu}\right\rangle$. We proceed as we did in (2.19)-(2.22) to compute $\left\|\xi_{v}\right\|^{2}$. With $\mathcal{K} a_{r+v}^{*} a_{-r}^{*} a_{-v}^{*}=a_{r+v}^{*} a_{-r}^{*} a_{-v}^{*}(\mathcal{K}+(r+$ $v)^{2}+r^{2}+v^{2}$ ) we obtain

$$
\begin{aligned}
\left\langle\xi_{v}, \mathcal{K} \xi_{v}\right\rangle=\sum_{m \geq 1} \frac{1}{2^{m}(m-1) !} \frac{1}{N^{m}} \sum_{\substack{v_{1} \in P_{S}, r_{1} \in P_{H}: \\
r_{1}+v_{1} \in P_{H}}} \cdots \sum_{\substack{v_{m} \in P_{S}, r_{m} \in P_{H}: \\
r_{m}+v_{m} \in P_{H}}} \theta\left(\left\{r_{j}, v_{j}\right\}_{j=1}^{m}\right) \\
\times\left[r_{m}^{2}+v_{m}^{2}+\left(r_{m}+v_{m}\right)^{2}\right] \prod_{i=1}^{m}\left(\eta_{r_{i}}+\eta_{r_{i}+v_{i}}\right)^{2} \sigma_{v_{i}}^{2}
\end{aligned}
$$

with the cutoff $\theta$ introduced in (2.18). Since all terms are positive, we can find an upper bound for $\left\langle\xi_{v}, \mathcal{K} \xi_{v}\right\rangle$ by replacing $\theta\left(\left\{r_{j}, v_{j}\right\}_{j=1}^{m}\right)$ with $\theta\left(\left\{r_{j}, v_{j}\right\}_{j=1}^{m-1}\right)$, removing conditions involving momenta with index $m$. Recalling (2.22), we find

$$
\begin{aligned}
\left\langle\xi_{v}, \mathcal{K} \xi_{v}\right\rangle & \leq \frac{1}{2 N} \sum_{\substack{v \in P_{S}, r \in P_{H} \\
r+v \in P_{H}}}\left[r^{2}+v^{2}+(r+v)^{2}\right]\left(\eta_{r}+\eta_{r+v}\right)^{2} \sigma_{v}^{2}\left\|\xi_{v}\right\|^{2} \\
& \leq \frac{2}{N} \sum_{\substack{v \in P_{S}, r \in P_{H} \\
r^{2}}} \eta_{r}\left(\eta_{r}+\eta_{r+v}\right) \sigma_{v}^{2}\left\|\xi_{v}\right\|^{2}+\mathcal{E}
\end{aligned}
$$

with (using Lemma 2.4 and the assumption $3 \kappa-2+4 \varepsilon<0$ )

$$
\begin{aligned}
\frac{\mathcal{E}}{\left\|\xi_{v}\right\|^{2}}= & \frac{2}{N} \sum_{\substack{v \in P_{S}, r \in P_{H} \\
r+v \in P_{H}}}\left(v^{2}+r \cdot v\right) \eta_{r}\left(\eta_{r}+\eta_{r+v}\right) \sigma_{v}^{2} \\
& \leq \frac{C}{N}\left(\left\|\sigma_{S}\right\|_{H^{1}}^{2}\left\|\eta_{H}\right\|^{2}+\left\|\sigma_{S}\right\|\left\|\eta_{H}\right\|\left\|\sigma_{S}\right\|_{H^{1}}\left\|\eta_{H}\right\|_{H^{1}}\right) \leq C N^{4 \kappa-1+\varepsilon} \leq C N^{5 \kappa / 2-\varepsilon} .
\end{aligned}
$$

This proves (5.1). In particular, (5.1) implies, together with Lemma 2.4, that

$$
\frac{\left\langle\xi_{v}, \mathcal{K} \xi_{v}\right\rangle}{\left\|\xi_{v}\right\|^{2}} \leq C N^{-1}\left\|\eta_{H}\right\|_{H^{1}}^{2}\left\|\sigma_{S}\right\|^{2} \leq C N^{5 \kappa / 2}
$$


which shows (2.24) with $j=1$ in Proposition 2.2.

Analogously, we find

$$
\begin{aligned}
\left\langle\xi_{v}, \mathcal{K N} \xi_{v}\right\rangle \leq \sum_{m \geq 1} \frac{3 m}{2^{m}(m-1) !} \frac{1}{N^{m}} \sum_{\substack{v_{1} \in P_{S}, r_{1} \in P_{H}: \\
r_{1}+v_{1} \in P_{H}}} \ldots \sum_{\substack{v_{m} \in P_{S}, r_{m} \in P_{H} \\
r_{m}+v_{m} \in P_{H}}} \theta\left(\left\{r_{j}, v_{j}\right\}_{j=1}^{m}\right) \\
\times\left[r_{m}^{2}+v_{m}^{2}+\left(r_{m}+v_{m}\right)^{2}\right] \prod_{i=1}^{m}\left(\eta_{r_{i}}+\eta_{r_{i}+v_{i}}\right)^{2} \sigma_{v_{i}}^{2} .
\end{aligned}
$$

Writing $m=1+(m-1)$ and bounding $\theta\left(\left\{r_{j}, v_{j}\right\}_{j=1}^{m}\right)$ by $\theta\left(\left\{r_{j}, v_{j}\right\}_{j=1}^{m-2}\right)$, we obtain

$$
\begin{aligned}
&\left\langle\xi_{v}, \mathcal{K N} \xi_{v}\right\rangle \leq 3\left\langle\xi_{v}, \mathcal{K} \xi_{v}\right\rangle+\frac{3}{4 N^{2}} \sum_{r, r^{\prime} \in P_{H}, v, v^{\prime} \in P_{S}}\left[r^{2}+v^{2}+(r+v)^{2}\right] \\
& \times\left(\eta_{r}+\eta_{r+v}\right)^{2}\left(\eta_{r^{\prime}}+\eta_{r^{\prime}+v^{\prime}}\right)^{2} \sigma_{v}^{2} \sigma_{v^{\prime}}^{2}\left\|\xi_{v}\right\|^{2}
\end{aligned}
$$

With (5.4) and with the bounds for $\left\|\eta_{H}\right\|_{H^{1}}^{2},\left\|\eta_{H}\right\|^{2},\left\|\sigma_{S}\right\|^{2}$ from Lemma 2.4, we find

$$
\frac{\left\langle\xi_{v}, \mathcal{K N} \xi_{v}\right\rangle}{\left\|\xi_{v}\right\|^{2}} \leq C N^{5 \kappa / 2} \cdot N^{9 \kappa / 2-2+\varepsilon}
$$

which shows (2.24) with $j=2$.

To show (2.23) we observe that, by (2.15), the operator $A_{v}$ only creates particles with momenta in $P_{S} \cup P_{H}$ and for each particle with momentum in $P_{S}$, it creates two particles with momenta in $P_{H}$. Since $|p|>N^{1-\kappa-\varepsilon}$ for all $p \in P_{H}$, we find, by (5.4),

$$
\begin{aligned}
\left\langle\xi_{v}, \mathcal{N} \xi_{v}\right\rangle & =\sum_{p \in P_{S} \cup P_{H}}\left\langle\xi_{v}, a_{p}^{*} a_{p} \xi_{v}\right\rangle=\frac{3}{2} \sum_{p \in P_{H}}\left\langle\xi_{v}, a_{p}^{*} a_{p} \xi_{v}\right\rangle \\
& \leq C N^{-2+2 \kappa+2 \varepsilon}\left\langle\xi_{v}, \mathcal{K} \xi_{v}\right\rangle \leq N^{9 \kappa / 2-2+2 \varepsilon}\left\|\xi_{v}\right\|^{2}
\end{aligned}
$$

proving (2.23) for $j=1$. Analogously, we find

$$
\begin{aligned}
\left\langle\xi_{v}, \mathcal{N}^{2} \xi_{\nu}\right\rangle & =\sum_{p \in P_{S} \cup P_{H}}\left\langle\mathcal{N}^{1 / 2} \xi_{v}, a_{p}^{*} a_{p} \mathcal{N}^{1 / 2} \xi_{v}\right\rangle \\
& \leq \frac{3}{2} \sum_{p \in P_{H}}\left\langle\mathcal{N}^{1 / 2} \xi_{v}, a_{p}^{*} a_{p} \mathcal{N}^{-1 / 2} \xi_{v}\right\rangle \leq C N^{-2+2 \kappa+2 \varepsilon}\left\langle\xi_{v}, \mathcal{K} \mathcal{N} \xi_{v}\right\rangle
\end{aligned}
$$

By (5.5), we obtain (2.23) with $j=2$. This completes the proof of Proposition 2.2.

\subsection{Expectation of the cubic term}

The goal of this section is to show (5.2). From (3.5), we have (using the reality of $\eta_{p}, \gamma_{p}, \sigma_{p}$ )

$$
\begin{aligned}
& \left\langle\xi_{v}, \mathcal{C}_{N} \xi_{v}\right\rangle \\
& =2 \frac{\sqrt{N_{0}}}{N} \sum_{m \geq 1} \frac{1}{m !(m-1) !} \sum_{\substack{p, r \in P_{H} \\
p+r \in P_{S}}} N^{\kappa} \widehat{V}\left(r / N^{1-\kappa}\right) \sigma_{p+r} \gamma_{p} \gamma_{r}\left\langle A_{v}^{m} \xi_{v}, a_{p+r}^{*} a_{-p}^{*} a_{-r}^{*} A_{v}^{m-1} \xi_{v}\right\rangle
\end{aligned}
$$


Proceeding as in the previous section, we get

$$
\begin{aligned}
& \left\langle\xi_{v}, \mathcal{C}_{N} \xi_{v}\right\rangle \\
& =2 \sqrt{\frac{N_{0}}{N}} \sum_{m \geq 1} \frac{1}{2^{m-1}(m-1) !} \frac{1}{N^{m}} \sum_{\substack{v_{1} \in P_{S}, r_{1} \in P_{H}: \\
r_{1}+v_{1} \in P_{H}}} \ldots \sum_{\substack{v_{m} \in P_{S}, r_{m} \in P_{H}: \\
r_{m}+v_{m} \in P_{H}}} \theta\left(\left\{r_{j}, v_{j}\right\}_{j=1}^{m}\right) \\
& \quad \times N^{\kappa} \widehat{V}\left(r_{m} / N^{1-\kappa}\right)\left(\eta_{r_{m}}+\eta_{r_{m}+v_{m}}\right) \gamma_{r_{m}} \gamma_{r_{m}+v_{m}} \sigma_{v_{m}}^{2} \prod_{i=1}^{m-1}\left(\eta_{r_{i}}+\eta_{r_{i}+v_{i}}\right)^{2} \sigma_{v_{i}}^{2} .
\end{aligned}
$$

To reconstruct the norm $\left\|\xi_{v}\right\|^{2}$ on the right-hand side, we need to free the momenta with index $m$. To this end, we recall the defintion (2.18) to write

$$
\theta\left(\left\{r_{j}, v_{j}\right\}_{j=1}^{m}\right)=\theta\left(\left\{r_{j}, v_{j}\right\}_{j=1}^{m-1}\right) \theta_{m}\left(\left\{r_{j}, v_{j}\right\}_{j=1}^{m}\right)
$$

with

$$
\theta_{m}\left(\left\{r_{j}, v_{j}\right\}_{j=1}^{m}\right)=\prod_{i, j=1}^{m-1} \prod_{\substack{p_{i}, p_{j}, p_{m}: \\ p_{\ell} \in\left\{-r_{\ell}, r_{\ell}+v_{\ell}\right\}}} \delta_{p_{i} \neq-p_{j}+v_{m}} \delta_{-p_{m}+v_{i} \neq p_{j}}
$$

collecting all conditions involving $\left\{r_{m}, v_{m}\right\}$. Writing $\theta_{m}=1+\left[\theta_{m}-1\right]$, we split $\left\langle\xi_{v}, \mathcal{C}_{N} \xi_{v}\right\rangle=I_{\mathcal{C}}+J_{\mathcal{C}}$ with (recall the expression (2.22) for $\left\|\xi_{v}\right\|^{2}$ )

$$
I_{\mathcal{C}}=2 \sqrt{\frac{N_{0}}{N}} \sum_{\substack{v \in P_{S}, r \in P_{H} \\ r+v \in P_{H}}} N^{\kappa-1} \widehat{V}\left(r / N^{1-\kappa}\right)\left(\eta_{r}+\eta_{r+v}\right) \gamma_{r} \gamma_{r+v} \sigma_{v}^{2}\left\|\xi_{v}\right\|^{2}
$$

and

$$
\begin{aligned}
& J_{\mathcal{C}}= 2 \sqrt{\frac{N_{0}}{N}} \sum_{m \geq 1} \frac{1}{2^{m-1}(m-1) !} \frac{1}{N^{m}} \sum_{\substack{v_{1} \in P_{S}, r_{1} \in P_{H} \\
r_{1}+v_{1} \in P_{H}}} \cdots \sum_{\substack{v_{m} \in P_{S}, r_{m} \in P_{H} \\
r_{m}+v_{m} \in P_{H}}} \theta\left(\left\{r_{j}, v_{j}\right\}_{j=1}^{m-1}\right) \\
& \times\left[\theta_{m}\left(\left\{r_{j}, v_{j}\right\}_{j=1}^{m}\right)-1\right] N^{\kappa} \widehat{V}\left(r_{m} / N^{1-\kappa}\right)\left(\eta_{r_{m}}+\eta_{r_{m}+v_{m}}\right) \gamma_{r_{m}} \gamma_{r_{m}+v_{m}} \sigma_{v_{m}}^{2} \\
& \times \prod_{i=1}^{m-1}\left(\eta_{r_{i}}+\eta_{r_{i}+v_{i}}\right)^{2} \sigma_{v_{i}}^{2} .
\end{aligned}
$$

With $\left|\sqrt{N_{0} / N}-1\right| \leq C\left\|\sigma_{L}\right\|^{2} / N$ and $\left|\gamma_{r} \gamma_{r+v}-1\right| \leq C N^{2 \kappa} /|r|^{4}$ for all $r \in P_{H}, v \in P_{S}$, we obtain (using (3.12) and the assumption $3 \kappa-2+4 \varepsilon<0$ ) that

$$
\frac{I_{\mathcal{C}}}{\left\|\xi_{v}\right\|^{2}} \leq \frac{2}{N} \sum_{\substack{v \in P_{S}, r \in P_{H}: \\ r+v \in P_{H}}} N^{\kappa} \widehat{V}\left(r / N^{1-\kappa}\right)\left(\eta_{r}+\eta_{r+v}\right) \sigma_{v}^{2}+C N^{5 \kappa / 2-\varepsilon} .
$$

To complete the proof of (5.2), we focus now on the error term $J_{\mathcal{C}}$. We observe that

$$
\begin{aligned}
\left|\theta_{m}\left(\left\{r_{j}, v_{j}\right\}_{j=1}^{m}\right)-1\right| \leq & \sum_{j=1}^{m-1}\left[\delta_{v_{j}, v_{m}}+\sum_{\substack{p_{m} \in\left\{-r_{m}, r_{m}+v_{m}\right\} \\
p_{j} \in\left\{-r_{j}, r_{j}+v_{j}\right\}}} \delta_{p_{m}, p_{j}}\right] \\
& +\sum_{j, k=1}^{m-1}\left[\sum_{\substack{p_{j} \in\left\{-r_{j}, r_{j}+v_{j}\right\} \\
p_{k} \in\left\{-r_{k}, r_{k}+v_{k}\right\}}} \delta_{v_{m}, p_{j}+p_{k}}+\sum_{\substack{p_{m} \in\left\{-r_{m}, r_{m}+v_{m}\right\} \\
p_{j} \in\left\{-r_{j}, r_{j}+v_{j}\right\}}} \delta_{p_{m},-p_{j}+v_{k}}\right] .
\end{aligned}
$$


We bound $\left|J_{\mathcal{C}}\right| \leq \mathrm{X}_{1}+\mathrm{X}_{2}$, with $\mathrm{X}_{1}$ denoting the contribution arising from the first term on the righthand side of (5.8) (this term involves two indices, $m$ and $j$ ) and $\mathrm{X}_{2}$ indicating the contribution from the second term on the right-hand side of (5.8) (this term involves three indices, $m, j, k$ ). We can estimate

$$
\begin{aligned}
\mathrm{X}_{1} \leq & C \sum_{m \geq 2} \frac{1}{2^{m-2}(m-2) !} \frac{1}{N^{m}} \sum_{\substack{v_{1} \in P_{S}, r_{1} \in P_{H}: \\
r_{1}+v_{1} \in P_{H}}} \cdots \sum_{\substack{v_{m} \in P_{S}, r_{m} \in P_{H} \\
r_{m}+v_{m} \in P_{H}}} \theta\left(\left\{r_{j}, v_{j}\right\}_{j=1}^{m-1}\right) \\
& \times N^{\kappa}\left|\widehat{V}\left(r_{m} / N^{1-\kappa}\right)\right|\left|\eta_{r_{m}}+\eta_{r_{m}+v_{m}}\right|\left|\gamma_{r_{m}}\right|\left|\gamma_{r_{m}+v_{m}}\right| \sigma_{v_{m}}^{2} \prod_{i=1}^{m-1}\left(\eta_{r_{i}}+\eta_{r_{i}+v_{i}}\right)^{2} \sigma_{v_{i}}^{2} \\
& \times\left[\delta_{v_{m}, v_{m-1}}+\sum_{\substack{p_{m-1}, p_{m}: \\
p_{\ell} \in\left\{-r_{\ell}, r_{\ell}+v_{\ell}\right\}}} \delta_{p_{m}, p_{m-1}}\right] .
\end{aligned}
$$

With $\theta\left(\left\{r_{j}, v_{j}\right\}_{j=1}^{m-1}\right) \leq \theta\left(\left\{r_{j}, v_{j}\right\}_{j=1}^{m-2}\right)$, we reconstruct $\left\|\xi_{v}\right\|^{2}$. Since $\left\|\gamma_{H}\right\|_{\infty} \leq C$, we end up with

$$
\begin{aligned}
\frac{\mathrm{X}_{1}}{\left\|\xi_{v}\right\|^{2}} \leq \frac{C}{N^{2}} \sum_{r, r^{\prime} \in P_{H}, v, v^{\prime} \in P_{S}} N^{\kappa}\left|\widehat{V}\left(r / N^{1-\kappa}\right)\right| \eta_{r}+\eta_{r+v} \| \eta_{r^{\prime}}+\left.\eta_{r^{\prime}+v^{\prime}}\right|^{2} \sigma_{v}^{2} \sigma_{v^{\prime}}^{2} \\
\times\left[\delta_{v, v^{\prime}}+\sum_{\substack{p \in\{-r, r+v\} \\
p^{\prime} \in\left\{-r^{\prime}, r^{\prime}+v^{\prime}\right\}}} \delta_{p, p^{\prime}}\right] \\
\leq C N^{2 \kappa-2}\left\|\sigma_{S}\right\|_{\infty}^{2}\left\|\sigma_{S}\right\|^{2}\left\|\eta_{H}\right\|^{2} \sum_{r \in P_{H}} \frac{\left|\widehat{V}\left(r / N^{1-\kappa}\right)\right|}{r^{2}}+C N^{4 \kappa-2}\left\|\sigma_{S}\right\|^{4} \sum_{r \in P_{H}}|r|^{-6} \\
\leq C N^{11 \kappa / 2-2+2 \varepsilon}+C N^{10 \kappa-5+3 \varepsilon} \leq C N^{5 \kappa / 2-\varepsilon},
\end{aligned}
$$

where we used Lemma 2.4, (3.12), the assumption $3 \kappa-2+4 \varepsilon<0$ and the remark that $\left|\eta_{r+v}\right| \leq$ $C N^{\kappa}|r|^{-2}$, for all $r \in P_{H}$ and $v \in P_{S}$. We can proceed similarly to estimate $X_{2}$. In the second term on the right-hand side of $(5.8)$, we have to sum over $(m-1)(m-2) / 2$ pairs of indices $j, k$. With $\theta\left(\left\{r_{j}, v_{j}\right\}_{j=1}^{m-1}\right) \leq \theta\left(\left\{r_{j}, v_{j}\right\}_{j=1}^{m-3}\right)$ and again with Lemma 2.4 and(3.12), we arrive at

$$
\begin{aligned}
\frac{\mathrm{X}_{2}}{\left\|\xi_{v}\right\|^{2}} \leq & \frac{C}{N^{3}} \sum_{\substack{r, r^{\prime}, r^{\prime \prime} \in P_{H}, v, v^{\prime}, v^{\prime \prime} \in P_{S}}} N^{\kappa}\left|\widehat{V}\left(r / N^{1-\kappa}\right)\right| \eta_{r}+\eta_{r+v} \| \eta_{r^{\prime}}+\left.\eta_{r^{\prime}+v^{\prime}}\right|^{2}\left|\eta_{r^{\prime \prime}}+\eta_{r^{\prime \prime}+v^{\prime \prime}}\right|^{2} \sigma_{v}^{2} \sigma_{v^{\prime}}^{2} \sigma_{v^{\prime \prime}}^{2} \\
& \times\left[\sum_{\substack{p \in\{-r, r+v\}, p^{\prime} \in\left\{-r^{\prime}, r^{\prime}+v^{\prime}\right\}}} \delta_{p,-p^{\prime}+v^{\prime \prime}}+\sum_{\substack{p^{\prime} \in\left\{-r^{\prime}, r^{\prime}+v^{\prime}\right\}, p^{\prime \prime} \in\left\{-r^{\prime \prime}, r^{\prime \prime}+v^{\prime \prime}\right\}}} \delta_{v, p^{\prime}+p^{\prime \prime}}\right] \\
& \leq C N^{4 \kappa-3}\left\|\sigma_{S}\right\|^{6}\left\|\eta_{H}\right\|^{2} \sum_{r \in P_{H}}|r|^{-6}+C N^{6 \kappa-3}\left\|\sigma_{S}\right\|^{6} \sum_{r \in P_{H}} \frac{\left|\widehat{V}\left(r / N^{1-\kappa}\right)\right|}{r^{2}} \sum_{r^{\prime} \in P_{H}}\left|r^{\prime}\right|^{-8} \\
& \leq C N^{29 \kappa / 2-7+5 \varepsilon} \leq C N^{5 \kappa / 2} \cdot N^{12 \kappa-7+5 \varepsilon} .
\end{aligned}
$$

Thus, $\left|J_{\mathcal{C}}\right| /\left\|\xi_{v}\right\|^{2} \leq N^{5 \kappa / 2} \cdot \max \left\{N^{-\varepsilon}, N^{12 \kappa-7+5 \varepsilon}\right\}$. With (5.7), this implies (5.2). 


\subsection{Expectation of the quartic term}

In this section we show the bound (5.3) for the expectation of $\mathcal{V}_{N}^{(H)}$. Pairing momenta in $P_{S}$, as we did in (2.20) and in the previous subsections, we obtain

$$
\begin{aligned}
& \left\langle\xi_{v}, \mathcal{V}_{N}^{(H)} \xi_{v}\right\rangle=\frac{1}{2 N} \sum_{m \geq 1} \frac{1}{m !} \frac{1}{N^{m}} \sum_{\substack{v_{1} \in P_{S}, r_{1}, \tilde{r}_{1} \in P_{H}: \\
r_{1}+v_{1}, \tilde{r}_{1}+v_{1} \in P_{H}}} \ldots \sum_{\substack{v_{m} \in P_{S}, r_{m}, \tilde{r}_{m} \in P_{H} \\
r_{m}+v_{m}, \tilde{r}_{m}+v_{m} \in P_{H}}} \\
& \times \theta\left(\left\{r_{j}, v_{j}\right\}_{j=1}^{m}\right) \theta\left(\left\{\tilde{r}_{j}, v_{j}\right\}_{j=1}^{m}\right) \prod_{i=1}^{m} \eta_{r_{i}} \eta_{\tilde{r}_{i}} \sigma_{\substack{v_{i} \\
r \in \Lambda^{*}, p, q \in P_{H} \\
p+r, q+r \in P_{H}}} N^{\kappa} \widehat{V}\left(r / N^{1-\kappa}\right) \\
& \times\left\langle\Omega, A_{r_{1}, v_{1}} \ldots A_{r_{m}, v_{m}} a_{p+r}^{*} a_{q}^{*} a_{p} a_{q+r} A_{\tilde{r}_{1}, v_{1}}^{*} \ldots A_{\tilde{r}_{m}, v_{m}}^{*} \Omega\right\rangle
\end{aligned}
$$

where we use the notation $A_{r_{i}, v_{i}}=a_{r_{i}+v_{i}} a_{-r_{i}}$ that was already introduced in (2.20). Next we observe that because of the cutoffs $\theta\left(\left\{r_{j}, v_{j}\right\}_{j=1}^{m}\right)$ and $\theta\left(\left\{\tilde{r}_{j}, v_{j}\right\}_{j=1}^{m}\right)$, at most two indices $i, j \in\{1, \ldots, m\}$ can be involved in contractions with the observable $a_{p+r}^{*} a_{q}^{*} a_{p} a_{q+r}$. We distinguish two possible cases:

1) There exists an index $i \in\{1, \ldots, m\}$ such that $a_{p}, a_{q+r}$ are contracted with $A_{\tilde{r}_{i}, v_{i}}^{*}$ and $a_{q}^{*}, a_{p+r}^{*}$ are contracted with $A_{r_{i}, v_{i}}$.

2) There are two indices $i \neq j \in\{1, \ldots, m\}$ such that the operators $a_{p}$ and $a_{q+r}$ are contracted with $a_{\tilde{p}_{i}}^{*}$ and $a_{\tilde{p}_{j}}^{*}$ for some $\tilde{p}_{\ell} \in\left\{-\tilde{r}_{\ell}, \tilde{r}_{\ell}+v_{\ell}\right\}, \ell=i, j$ and the operators $a_{q}^{*}, a_{p+r}^{*}$ are contracted with $a_{p_{i}}, a_{p_{j}}$, with $p_{\ell} \in\left\{-r_{\ell}, r_{\ell}+v_{\ell}\right\}, \ell=i, j$. Note that in this case the operators $a_{-\tilde{p}_{i}+v_{i}}^{*}, a_{-\tilde{p}_{j}+v_{j}}^{*}$ have to be contracted with $a_{-p_{i}+v_{i}}, a_{-p_{j}+v_{j}}$.

We denote by $\mathrm{V}_{1}$ and $\mathrm{V}_{2}$ the contributions to $\left\langle\xi_{v}, \mathcal{V}_{N}^{(H)} \xi_{v}\right\rangle$ arising from the two cases described above. Let us first consider $\mathrm{V}_{1}$. There are $m$ choices (all leading to the same contribution) for the index $i \in\{1, \ldots, m\}$ labelling momenta to be contracted with the observable. Let us fix $i=m$. Then we have $p=\tilde{p}_{m}, q+r=-\tilde{p}_{m}+v_{m}$ with $\tilde{p}_{m} \in\left\{-\tilde{r}_{m}, \tilde{r}_{m}+v_{m}\right\}$ and $p+r=p_{m}, q=-p_{m}+v_{m}$ with $p_{m} \in\left\{-r_{m}, r_{m}+v_{m}\right\}$. Note that the choice of $p$ and $p+r$ also determines $q$ and $q+r$, since we always have $q=v_{m}-(p+r)$. The presence of the cutoffs immediately implies that $A_{r_{j}, v_{j}}$ is fully contracted with $A_{\tilde{r}_{j}, v_{j}}^{*}$, for all $j \neq m$. We find

$$
\begin{aligned}
& \left\langle\xi_{v}, \mathrm{~V}_{1} \xi_{v}\right\rangle \\
& =\frac{1}{2 N} \sum_{m \geq 1} \frac{1}{(m-1) !} \frac{1}{N^{m}} \sum_{\substack{v_{1} \in P_{S}, r_{1}, \tilde{r}_{1} \in P_{H}: \\
r_{1}+v_{1}, \tilde{r}_{1}+v_{1} \in P_{H}}} \ldots \sum_{\substack{v_{m} \in P_{S}, r_{m}, \tilde{r}_{m} \in P_{H}: \\
r_{m}+v_{m}, \tilde{r}_{m}+v_{m} \in P_{H}}} \theta\left(\left\{r_{j}, v_{j}\right\}_{j=1}^{m}\right) \theta\left(\left\{\tilde{r}_{j}, v_{j}\right\}_{j=1}^{m}\right) \\
& \times \prod_{j=1}^{m-1} \eta_{r_{j}} \eta_{\tilde{r}_{j}}\left(\delta_{r_{j}, \tilde{r}_{j}}+\delta_{-r_{j}, \tilde{r}_{j}+v_{j}}\right) \sigma_{v_{j}}^{2} \eta_{r_{m}} \eta_{\tilde{r}_{m}} \sigma_{\substack{v_{m} \\
r \in \Lambda^{*}, p \in P_{H}: \\
p-v_{m}, p+r \in P_{H}}} \sum_{\substack{p_{m} \in\left\{-r_{m}, r_{m}+v_{m}\right\} \\
\tilde{p}_{m} \in\left\{-\tilde{r}_{m}, \tilde{r}_{m}+v_{m}\right\}}} N^{\kappa} \widehat{V}\left(r / N^{1-\kappa}\right) \sum_{p} \delta_{p, p_{m}} \delta_{p+r}, \tilde{p}_{m} .
\end{aligned}
$$

Since here (in contrast to the previous subsections) the contraction does not fix $\tilde{r}_{m}$ to be either $r_{m}$ or $-\left(r_{m}+v_{m}\right)$, we cannot erase the cutoff $\theta\left(\left\{\tilde{r}_{j}, v_{j}\right\}_{j=1}^{m}\right)$. With the decomposition (5.6), we can replace, on the right-hand side of (5.10),

$$
\theta\left(\left\{r_{j}, v_{j}\right\}_{j=1}^{m}\right) \theta\left(\left\{\tilde{r}_{j}, v_{j}\right\}_{j=1}^{m}\right)=\theta\left(\left\{r_{j}, v_{j}\right\}_{j=1}^{m-1}\right) \theta_{m}\left(\left\{r_{j}, v_{j}\right\}_{j=1}^{m}\right) \theta_{m}\left(\left\{\tilde{r}_{j}, v_{j}\right\}_{j=1}^{m}\right) .
$$

Writing

$$
\theta_{m}\left(\left\{\tilde{r}_{j}, v_{j}\right\}_{j=1}^{m}\right) \theta_{m}\left(\left\{\tilde{r}_{j}, v_{j}\right\}_{j=1}^{m}\right)=1+\left[\theta_{m}\left(\left\{r_{j}, v_{j}\right\}_{j=1}^{m}\right) \theta_{m}\left(\left\{\tilde{r}_{j}, v_{j}\right\}_{j=1}^{m}\right)-1\right]
$$


we split (as we did in the last subsection) $\left\langle\xi_{v}, \mathrm{~V}_{1} \xi_{v}\right\rangle=I_{\mathcal{V}}+J_{\mathcal{V}}$, with

$$
I_{\mathcal{V}}=\frac{1}{N^{2}} \sum_{r \in \Lambda^{*}} \sum_{\substack{v \in P_{S}, p \in P_{H}: \\ p+r, p-v, p+r-v \in P_{H}}} N^{\kappa} \widehat{V}\left(r / N^{1-\kappa}\right) \eta_{p}\left(\eta_{p+r}+\eta_{p+r-v}\right) \sigma_{v}^{2}\left\|\xi_{v}\right\|^{2}
$$

and

$$
\begin{aligned}
& J_{\mathcal{V}}=\frac{1}{2 N} \sum_{m \geq 1} \frac{1}{2^{m-1}(m-1) !} \frac{1}{N^{m}} \sum_{\substack{v_{1} \in P_{S}, r_{1} \in P_{H}: \\
r_{1}+v_{1} \in P_{H}}} \cdots \sum_{\substack{v_{m-1} \in P_{S}, r_{m-1} \in P_{H}: v_{m} \in P_{S}, r_{m}, \tilde{r}_{m} \in P_{H} \\
r_{m-1}+v_{m-1} \in P_{H}}} \sum_{r_{m}+v_{m}, \tilde{r}_{m}+v_{m} \in P_{H}} \\
& \times \theta\left(\left\{r_{j}, v_{j}\right\}_{j=1}^{m-1}\right)\left[\theta_{m}\left(\left\{r_{j}, v_{j}\right\}_{j=1}^{m}\right) \theta_{m}\left(\left\{r_{j}^{\#}, v_{j}\right\}_{j=1}^{m}\right)-1\right] \prod_{i=1}^{m-1}\left(\eta_{r_{i}}+\eta_{r_{i}+v_{i}}\right)^{2} \sigma_{v_{i}}^{2} \\
& \times \eta_{r_{m}} \eta_{\tilde{r}_{m}} \sigma_{v_{m}}^{2} \sum_{r \in \Lambda^{*}} N^{\kappa} \widehat{V}\left(r / N^{1-\kappa}\right) \sum_{\substack{p \in P_{H}: p+r, p-v_{m}, p+r-v_{m} \in P_{H}}} \sum_{\substack{p_{m} \in\left\{-r_{m}, r_{m}+v_{m}\right\} \\
\tilde{p}_{m} \in\left\{-\tilde{r}_{m}, \tilde{r}_{m}+v_{m}\right\}}} \delta_{p, p_{m}} \delta_{p+r, \tilde{p}_{m}}
\end{aligned}
$$

where $r_{j}^{\sharp}=r_{j}$ for $j=1, \ldots, m-1$ and $r_{m}^{\sharp}=\tilde{r}_{m}$ in the argument of $\theta_{m}$. Observing that, with Lemma 2.4 and (3.12),

$$
\begin{aligned}
& \frac{1}{N^{2}} \sum_{r \in \Lambda^{*}} \sum_{\substack{v \in P_{S}, p \in \Lambda^{*}: \\
p \in P_{H}^{c} \text { or } p-v \in P_{H}^{c}}} N^{\kappa}\left|\widehat{V}\left(r / N^{1-\kappa}\right)\right|\left|\eta_{p}\right|\left(\left|\eta_{p+r}\right|+\left|\eta_{p+r-v}\right|\right) \sigma_{v}^{2} \\
& \leq C N^{-2+2 \kappa}\left\|\sigma_{S}\right\|^{2}\left[\sum_{|p| \leq N^{1-\kappa-\varepsilon}}|p|^{-2}\right] \sup _{p \in \Lambda^{*}} \sum_{r \in \Lambda^{*}}\left|\widehat{V}\left(r / N^{1-\kappa}\right) \| \eta_{p+r}\right| \leq C N^{5 \kappa / 2-\varepsilon},
\end{aligned}
$$

we conclude from (5.11) (switching $p+r \rightarrow p$ and $v \rightarrow-v$ ) that

$$
\frac{I_{\mathcal{V}}}{\left\|\xi_{v}\right\|^{2}} \leq \frac{1}{N^{2}} \sum_{\substack{v \in P_{S}, p \in P_{H}: \\ p+v \in P_{H}}}\left(N^{\kappa} \widehat{V}\left(\cdot / N^{1-\kappa}\right) * \eta\right)_{p}\left(\eta_{p}+\eta_{p+v}\right) \sigma_{v}^{2}+C N^{5 \kappa / 2-\varepsilon}
$$

Let us now focus on the term $J_{\mathcal{V}}$. With

$$
\begin{aligned}
& \left|\theta_{m}\left(\left\{r_{j}, v_{j}\right\}_{j=1}^{m}\right) \theta_{m}\left(\left\{\tilde{r}_{j}, v_{j}\right\}_{j=1}^{m}\right)-1\right| \\
& \leq \sum_{j=1}^{m-1} \delta_{v_{m}, v_{j}}+\sum_{j=1}^{m-1}\left[\sum_{\substack{p_{j} \in\left\{-r_{j}, r_{j}+v_{j}\right\} \\
p_{m} \in\left\{-r_{m}, r_{m}+v_{m}\right\}}} \delta_{p_{m}, p_{j}}+\sum_{\substack{p_{j} \in\left\{-r_{j}, r_{j}+v_{j}\right\} \\
\tilde{p}_{m} \in\left\{-\tilde{r}_{m}, \tilde{r}_{m}+v_{m}\right\}}} \delta_{\tilde{p}_{m}, p_{j}}\right] \\
& \quad+\sum_{j, k=1}^{m-1}\left[\sum_{\substack{p_{j} \in\left\{-r_{j}, r_{j}+v_{j}\right\} \\
p_{m} \in\left\{-r_{m}, r_{m}+v_{m}\right\}}} \delta_{p_{m},-p_{j}+v_{k}}+\sum_{\substack{p_{j} \in\left\{-r_{j}, r_{j}+v_{j}\right\} \\
\tilde{p}_{m} \in\left\{-\tilde{r}_{m}, \tilde{r}_{m}+v_{m}\right\}}} \delta_{\tilde{p}_{m},-p_{j}+v_{k}}\right] \\
& \quad+\sum_{\substack{j, k=1 \\
j \neq k}} \sum_{\substack{p_{j} \in\left\{-r_{j}, r_{j}+v_{j}\right\} \\
p_{k} \in\left\{-r_{k}, r_{k}+v_{k}\right\}}} \delta_{v_{m}, p_{j}+p_{k}}
\end{aligned}
$$

we can bound $\left|J_{\mathcal{V}}\right| \leq \mathrm{W}_{1}+\mathrm{W}_{2}+\mathrm{W}_{3}+\mathrm{W}_{4}$, with $W_{\ell}$ indicating the contribution to (5.12) arising from the $\ell$ th term on the right-hand side of (5.14).

The term $\mathrm{W}_{1}$ contains the sum of $(m-1)$ identical contributions, corresponding to $j \in\{1, \ldots, m-1\}$ in the first term on the right-hand side of (5.14). Let us fix $j=m-1$. Estimating $\theta\left(\left\{r_{j}, v_{j}\right\}_{j=1}^{m-1}\right) \leq$ 
$\theta\left(\left\{r_{j}, v_{j}\right\}_{j=1}^{m-2}\right)$ and reconstructing the expression (2.22) for $\left\|\xi_{v}\right\|^{2}$, we can bound (the momenta $r^{\prime}, r^{\prime \prime}, \tilde{r}^{\prime \prime}$ correspond to $\left.r_{m-1}, r_{m}, \tilde{r}_{m}\right)$

$$
\frac{\mathrm{W}_{1}}{\left\|\xi_{v}\right\|^{2}} \leq C N^{-3} \sum_{r \in \Lambda^{*}} N^{\kappa}\left|\widehat{V}\left(r / N^{1-\kappa}\right)\right| \sum_{\substack{r^{\prime}, r^{\prime \prime}, \tilde{r}^{\prime \prime} \in P_{H} \\ v^{\prime} \in P_{S}}}\left(\eta_{r^{\prime}}+\eta_{r^{\prime}+v^{\prime}}\right)^{2}\left|\eta_{r^{\prime \prime}}\right|\left|\eta_{\tilde{r}^{\prime \prime}}\right| \sigma_{\substack{v^{\prime} \\ p^{\prime \prime} \in\left\{-r^{\prime \prime}, r^{\prime \prime}+v^{\prime}\right\} \\ \tilde{p}^{\prime \prime} \in\left\{-\tilde{r}^{\prime \prime}, \tilde{r}^{\prime \prime}+v^{\prime}\right\}}} \delta_{p^{\prime \prime}+r, \tilde{p}^{\prime \prime}}
$$

With Lemma 2.4 and with the estimate

$$
\sup _{v \in P_{S} \cup\{0\}} \frac{1}{N^{2}} \sum_{\substack{r \in \Lambda^{*}, q \in P_{H}: \\ q-r \in P_{H}}} N^{\kappa}\left|\widehat{V}\left(r / N^{1-\kappa}\right)\right|\left|\eta_{q-v}\right|\left|\eta_{q-r}\right| \leq C N^{\kappa},
$$

which can be shown similarly to (3.12) (using $V \in L^{q}\left(\mathbb{R}^{3}\right)$, for some $q>3 / 2$ ), we find

$$
\frac{\mathrm{W}_{1}}{\left\|\xi_{v}\right\|^{2}} \leq C N^{\kappa-1}\left\|\eta_{H}\right\|^{2}\left\|\sigma_{S}\right\|_{\infty}^{2}\left\|\sigma_{S}\right\|^{2} \leq C N^{11 \kappa / 2-2+2 \varepsilon} \leq C N^{5 \kappa / 2-\varepsilon}
$$

since $3 \kappa-2+4 \varepsilon<0$. Analogously, we bound, with (3.12) and Lemma 2.4,

$$
\begin{aligned}
& \frac{\mathrm{W}_{2}}{\left\|\xi_{v}\right\|^{2}} \leq C N^{-3} \sum_{r \in \Lambda^{*}} N^{\kappa}\left|\widehat{V}\left(r / N^{1-\kappa}\right)\right| \sum_{\substack{r^{\prime}, r^{\prime \prime}, \tilde{r}^{\prime \prime} \in P_{H} \\
v^{\prime}, v^{\prime \prime} \in P_{S}}}\left(\eta_{r^{\prime}}+\eta_{r^{\prime}+v^{\prime}}\right)^{2}\left|\eta_{r^{\prime \prime}}\right|\left|\eta_{\tilde{r}^{\prime \prime}}\right| \sigma_{v^{\prime}}^{2} \sigma_{v^{\prime \prime}}^{2} \\
& \times \sum_{\substack{p^{\prime \prime} \in\left\{-r^{\prime \prime}, r^{\prime \prime}+v^{\prime \prime}\right\} \\
\tilde{p}^{\prime \prime} \in\left\{-\tilde{r}^{\prime \prime}, \tilde{r}^{\prime \prime}+v^{\prime \prime}\right\}}} \delta_{p^{\prime \prime}+r, \tilde{p}^{\prime \prime}}\left[\sum_{\begin{array}{c}
p^{\prime} \in\left\{-r^{\prime}, r^{\prime}+v^{\prime}\right\} \\
p^{\prime \prime} \in\left\{-r^{\prime \prime}, r^{\prime \prime}+v^{\prime \prime}\right\}
\end{array}} \delta_{p^{\prime}, p^{\prime \prime}}+\sum_{\begin{array}{c}
p^{\prime} \in\left\{-r^{\prime}, r^{\prime}+v^{\prime}\right\} \\
\tilde{p}^{\prime \prime} \in\left\{-\tilde{r}^{\prime \prime}, \tilde{r}^{\prime \prime}+v^{\prime \prime}\right\}
\end{array}} \delta_{p^{\prime}, \tilde{p}^{\prime \prime}}\right] \\
& \leq C N^{-3+5 \kappa}\left\|\sigma_{S}\right\|^{4}\left[\sum_{r^{\prime} \in P_{H}}\left|r^{\prime}\right|^{-6}\right]\left[\sup _{r^{\prime} \in \Lambda^{*}} \sum_{r \in \Lambda^{*}, r \neq-r^{\prime}} \frac{\left|\widehat{V}\left(r / N^{1-\kappa}\right)\right|}{\left|r+r^{\prime}\right|^{2}}\right] \leq C N^{5 \kappa / 2-\varepsilon} \text {. }
\end{aligned}
$$

As for $\mathrm{W}_{3}$, there are $(m-1)(m-2)$ possible choices of the indices $j, k$ in $(5.14)$, all leading to the same contribution. We fix $j=m-1$ and $k=m-2$. Estimating now $\theta\left(\left\{r_{j}, v_{j}\right\}_{j=1}^{m-1}\right) \leq \theta\left(\left\{r_{j}, v_{j}\right\}_{j=1}^{m-3}\right)$, we obtain, with (3.12),

$$
\begin{aligned}
& \frac{\mathrm{W}_{3}}{\left\|\xi_{v}\right\|^{2}} \leq C N^{-4} \sum_{r \in \Lambda^{*}} N^{\kappa}\left|\widehat{V}\left(r / N^{1-\kappa}\right)\right| \\
& \times \sum_{\substack{r^{\prime}, r^{\prime \prime}, r^{\prime \prime \prime}, r^{\prime \prime \prime} \in P_{H} \\
v^{\prime}, v^{\prime \prime}, v^{\prime \prime \prime} \in P_{S}}}\left(\eta_{r^{\prime}}+\eta_{r^{\prime}+v^{\prime}}\right)^{2}\left(\eta_{r^{\prime \prime}}+\eta_{r^{\prime \prime}+v^{\prime \prime}}\right)^{2}\left|\eta_{r^{\prime \prime \prime}}\right|\left|\eta_{\tilde{r}^{\prime \prime \prime}}\right| \sigma_{v^{\prime}}^{2} \sigma_{v^{\prime \prime}}^{2} \sigma_{v^{\prime \prime \prime}}^{2} \\
& \times \sum_{\begin{array}{c}
p^{\prime \prime \prime} \in\left\{-r^{\prime \prime \prime}, r^{\prime \prime \prime}+v^{\prime \prime \prime}\right\} \\
\tilde{p}^{\prime \prime \prime} \in\left\{-\tilde{r}^{\prime \prime \prime}, \tilde{r}^{\prime \prime \prime}+v^{\prime \prime \prime}\right\}
\end{array}} \delta_{p^{\prime \prime \prime}+r, \tilde{p}^{\prime \prime \prime}}\left[\sum_{\begin{array}{c}
p^{\prime} \in\left\{-r^{\prime}, r^{\prime}+v^{\prime}\right\} \\
p^{\prime \prime \prime} \in\left\{-r^{\prime \prime \prime}, r^{\prime \prime \prime}+v^{\prime \prime \prime}\right\}
\end{array}} \delta_{p^{\prime \prime \prime},-p^{\prime}+v^{\prime \prime}}+\sum_{\begin{array}{c}
p^{\prime} \in\left\{-r^{\prime}, r^{\prime}+v^{\prime}\right\} \\
\tilde{p}^{\prime \prime \prime} \in\left\{-\tilde{r}^{\prime \prime \prime}, \tilde{r}^{\prime \prime \prime}+v^{\prime \prime \prime}\right\}
\end{array}} \delta_{\tilde{p}^{\prime \prime \prime},-p^{\prime}+v^{\prime \prime}}\right] \\
& \leq C N^{-3+4 \kappa}\left\|\sigma_{S}\right\|^{6}\left\|\eta_{H}\right\|^{2} \sum_{r^{\prime} \in P_{H}}\left|r^{\prime}\right|^{-6} \leq C N^{5 \kappa / 2} \cdot N^{12 \kappa-7+4 \varepsilon} \text {. }
\end{aligned}
$$


Analogously, with Lemma 2.4 and (5.15), we find

$$
\begin{aligned}
\frac{\mathrm{W}_{4}}{\left\|\xi_{v}\right\|^{2}} \leq & C N^{-4} \sum_{r \in \Lambda^{*}} N^{\kappa}\left|\widehat{V}\left(r / N^{1-\kappa}\right)\right| \\
& \times \sum_{\substack{r^{\prime}, r^{\prime \prime}, r^{\prime \prime \prime}, \tilde{r}^{\prime \prime \prime} \in P_{H} \\
v^{\prime}, v^{\prime \prime}, v^{\prime \prime \prime} \in P_{S}}}\left(\eta_{r^{\prime}}+\eta_{r^{\prime}+v^{\prime}}\right)^{2}\left(\eta_{r^{\prime \prime}}+\eta_{r^{\prime \prime}+v^{\prime \prime}}\right)^{2}\left|\eta_{r^{\prime \prime \prime}}\right|\left|\eta_{\tilde{r}^{\prime \prime \prime}}\right| \sigma_{v^{\prime}}^{2} \sigma_{v^{\prime \prime}}^{2} \sigma_{v^{\prime \prime \prime}}^{2} \\
& \times \sum_{\substack{p^{\prime \prime \prime} \in\left\{-r^{\prime \prime \prime} \in\left\{-r^{\prime \prime \prime}+v^{\prime \prime \prime}\right\} \\
\tilde{p}^{\prime \prime \prime}, \tilde{r}^{\prime \prime \prime}+v^{\prime \prime \prime}\right\}}} \delta_{p^{\prime \prime \prime}+r, \tilde{p}^{\prime \prime \prime}} \sum_{\substack{p^{\prime} \in\left\{-r^{\prime}, r^{\prime}+v^{\prime}\right\} \\
p^{\prime \prime} \in\left\{-r^{\prime \prime}, r^{\prime \prime}+v^{\prime \prime}\right\}}} \delta_{v^{\prime \prime \prime}, p^{\prime}+p^{\prime \prime}} \\
\leq & C N^{-2+5 \kappa}\left\|\sigma_{S}\right\|^{6} \sum_{r^{\prime} \in P_{H}}\left|r^{\prime}\right|^{-8} \leq C N^{5 \kappa / 2} \cdot N^{12 \kappa-7+5 \varepsilon} .
\end{aligned}
$$

Together with (5.16), (5.17), (5.18), we conclude that

$$
\left|J_{\mathcal{V}}\right| \leq C N^{5 \kappa / 2} \cdot \max \left\{N^{-\varepsilon}, N^{12 \kappa-7+5 \varepsilon}\right\} .
$$

Finally, we consider the term $\mathrm{V}_{2}$, associated with the second case listed after (5.9). We fix $i=m$ and $j=m-1$ and we consider all possible contractions of $a_{p}$ with $a_{\tilde{p}_{m}}^{*}$, of $a_{q+r}$ with $a_{\tilde{p}_{m-1}}^{*}$ and of $a_{q}^{*}, a_{p+r}^{*}$ with $a_{p_{m}}, a_{p_{m-1}}$, where $\tilde{p}_{\ell} \in\left\{-\tilde{r}_{\ell}, \tilde{r}_{\ell}+v_{\ell}\right\}$ and $p_{\ell} \in\left\{-r_{\ell}, r_{\ell}+v_{\ell}\right\}$, for $\ell=m, m-1$. We obtain

$$
\begin{aligned}
& \left\langle\xi_{v}, \mathrm{~V}_{2} \xi_{v}\right\rangle \\
& =\frac{1}{2 N} \sum_{m \geq 2} \frac{1}{(m-2) !} \frac{1}{N^{m}} \sum_{\substack{v_{1} \in P_{S}, r_{1}, \tilde{r}_{1} \in P_{H}: \\
r_{1}+v_{1}, \tilde{r}_{1}+v_{1} \in P_{H}}} \ldots \sum_{\substack{v_{m} \in P_{S}, r_{m}, \tilde{r}_{m} \in P_{H}: \\
r_{m}+v_{m}, \tilde{r}_{m}+v_{m} \in P_{H}}} \theta\left(\left\{r_{j}, v_{j}\right\}_{j=1}^{m}\right) \theta\left(\left\{\tilde{r}_{j}, v_{j}\right\}_{j=1}^{m}\right) \\
& \quad \times \prod_{i=1}^{m-2} \eta_{r_{i}} \eta_{\tilde{r}_{i}}\left(\delta_{\tilde{r}_{i}, r_{i}}+\delta_{-\tilde{r}_{i}, r_{i}+v_{i}}\right) \sigma_{v_{i}}^{2} \prod_{\substack{j=m, m-1 \\
\eta_{r_{j}}}} \eta_{\tilde{r}_{j}} \sigma_{v_{j}}^{2} \\
& \quad \times \sum_{\substack{r \in \Lambda^{*}, p, q \in P_{H} \\
p-r, q-r \in P_{H}}} N^{K} \widehat{V}\left(r / N^{1-\kappa}\right) \sum_{\substack{\tilde{p}_{\ell} \in\left\{\tilde{r}_{\ell}, \tilde{r}_{\ell}+v_{\ell}\right\} \\
\ell=m-1, m}} \delta_{p, \tilde{p}_{m}} \delta_{q+r, \tilde{p}_{m-1}} \\
& \quad \times \sum_{\substack{p_{\ell} \in\left\{-r_{\ell}, r_{\ell}+v_{\ell}\right\} \\
\ell=m-1, m}}\left(\delta_{q, p_{m}} \delta_{p+r, p_{m-1}}+\delta_{q, p_{m-1}} \delta_{p+r, p_{m}}\right)\left(\delta_{\tilde{p}_{m}, p_{m}}+\delta_{-\tilde{p}_{m}+v_{m},-p_{m-1}+v_{m-1}}\right) .
\end{aligned}
$$

Estimating $\theta\left(\left\{r_{j}, v_{j}\right\}_{j=1}^{m}\right) \theta\left(\left\{\tilde{r}_{j}, v_{j}\right\}_{j=1}^{m}\right) \leq \theta\left(\left\{r_{j}, v_{j}\right\}_{j=1}^{m-2}\right)$ and using Lemma 2.4 and the condition $3 \kappa-2+4 \varepsilon<0$, we find

$$
\begin{aligned}
& \frac{\left|\left\langle\xi_{v}, \mathrm{~V}_{2} \xi_{v}\right\rangle\right|}{\left\|\xi_{v}\right\|^{2}} \\
& \leq C N^{-3} \sum_{r \in \Lambda^{*}} N^{\kappa} \widehat{V}\left(r / N^{1-\kappa}\right) \sum_{\substack{r^{\prime}, \tilde{r}^{\prime}, r^{\prime \prime}, \tilde{r}^{\prime \prime} \in P_{H} \\
v^{\prime}, v^{\prime \prime} \in P_{S}}}\left|\eta_{r^{\prime}}\left\|\eta_{\tilde{r}^{\prime}}\right\| \eta_{r^{\prime \prime}} \| \eta_{\tilde{r}^{\prime \prime}}\right| \sigma_{v^{\prime}}^{2} \sigma_{v^{\prime \prime}}^{2} \\
& \times \sum_{\substack{p^{\prime} \in\left\{-r^{\prime}, r^{\prime}+v^{\prime}\right\} \\
p^{\prime \prime} \in\left\{-r^{\prime \prime}, r^{\prime \prime}+v^{\prime \prime}\right\}}} \sum_{\substack{\tilde{p}^{\prime} \in\left\{-\tilde{r}^{\prime}, \tilde{r}^{\prime \prime}+v^{\prime}\right\} \\
\tilde{p}^{\prime \prime} \in\left\{-\tilde{r}^{\prime \prime}, \tilde{r}^{\prime \prime}+v^{\prime \prime}\right\}}}\left(\delta_{\tilde{p}^{\prime}, p^{\prime \prime}+r} \delta_{\tilde{p}^{\prime \prime}+r, p^{\prime}}+\delta_{\tilde{p}^{\prime}, p^{\prime}+r} \delta_{\tilde{p}^{\prime \prime}+r, p^{\prime \prime}}\right) \\
& \times\left(\delta_{\tilde{p}^{\prime \prime}, p^{\prime \prime}}+\delta_{-\tilde{p}^{\prime \prime}+v^{\prime \prime},-p^{\prime}+v^{\prime}}\right) \\
& \leq C N^{-3+\kappa}\left\|\eta_{H}\right\|^{4}\left\|\sigma_{S}\right\|^{4} \leq C N^{10 \kappa-5+2 \varepsilon} \leq C N^{5 \kappa / 2-\varepsilon} \text {. }
\end{aligned}
$$

With (5.13) and (5.19), we obtain (5.3). 


\section{A. Proof of Proposition 1.2}

The proof of Proposition 1.2 is based on standard results, which are collected in this section for the reader's convenience. In particular, we follow [18] (see Lemma 2.1.3) and [20, Sec. 12] for Lemmas A.1 and A.2 and the proof of Lemma 3.3.2 in [1] for Lemma A.4 (control on the second moment of $\mathcal{N}$ allows us to avoid the condition imposed in [1] that $V$ is strictly positive around the origin).

The proof of Proposition 1.2 is divided into three parts. First, we show how to switch from periodic boundary conditions to Dirichlet boundary conditions, increasing the size of the box a bit. In the second step, we replicate the Dirichlet trial state obtained in the first step to obtain an upper bound on the energy in a sequence of boxes whose size increases to infinity (but with fixed density). In the last step, we show how to pass from the grand canonical to the canonical setting.

Let $\Psi_{L}=\left\{\Psi_{L}^{(n)}\right\}_{n \geq 0} \in \mathcal{F}\left(\Lambda_{L}\right)$ be a normalised trial state for the Fock-space Hamiltonian $\mathcal{H}$ defined on the box $\Lambda_{L}$ with periodic boundary conditions (in fact, we denote by $\Psi_{L}^{(n)}\left(x_{1}, \ldots, x_{n}\right)$ the $L$-periodic extension of $\Psi_{L}^{(n)}$ to the whole space $\left.\mathbb{R}^{3 n}\right)$. For $u \in \Lambda_{L}$, we define $\Psi_{L+2 b, u}^{D} \in \mathcal{F}\left(\Lambda_{L+2 b}^{u}\right)$, where $\Lambda_{L+2 b}^{u}=u+\Lambda_{L+2 b}$ is a box centred at $u$, with side length $L+2 b$, setting, for any $n \in \mathbb{N}$,

$$
\left(\Psi_{L+2 b, u}^{\mathrm{D}}\right)^{(n)}\left(x_{1}, \ldots, x_{n}\right)=\Psi_{L}^{(n)}\left(x_{1}, \ldots, x_{n}\right) \prod_{i=1}^{n} Q_{L, b}\left(x_{i}-u\right)
$$

where $Q_{L, b}\left(x_{i}\right)=\prod_{j=1}^{3} q_{L, b}\left(x_{i}^{(j)}\right)$ with $q_{L, b}: \mathbb{R} \rightarrow[0 ; 1]$ defined by

$$
q_{L, b}(t)= \begin{cases}\cos \left(\frac{\pi(t+L / 2-b)}{4 b}\right) & \text { if }\left|t+\frac{L}{2}\right| \leq b \\ 1 & \text { if }|t|<\frac{L}{2}-b \\ \cos \left(\frac{\pi(t-L / 2+b)}{4 b}\right) & \text { if }\left|t-\frac{L}{2}\right| \leq b \\ 0 & \text { otherwise. }\end{cases}
$$

By definition $\left(\Psi_{L+2 b, u}^{\text {Dir }}\right)^{(n)}$ satisfies Dirichlet boundary condition on the box $\Lambda_{L+2 b}^{u}$. The following lemma allows us to compare energy and moments of the number of particles of $\Psi_{L+2 b, u}^{\mathrm{D}}$ with those of $\Psi_{L}$.

Lemma A.1. Under the assumptions of Proposition 1.2, let $\Psi_{L+2 b, u}^{D}$ be defined as in (A.1) with $u \in \Lambda_{L}$. Then we have $\left\|\Psi_{L+2 b, u}^{\mathrm{D}}\right\|=1$. Moreover, for all $j \in \mathbb{N}$,

$$
\left\langle\Psi_{L+2 b, u}^{\mathrm{D}}, \mathcal{N}^{j} \Psi_{L+2 b, u}^{\mathrm{D}}\right\rangle=\left\langle\Psi_{L}, \mathcal{N}^{j} \Psi_{L}\right\rangle,
$$

and there exists $\bar{u} \in \Lambda_{L}$ such that

$$
\left\langle\Psi_{L+2 b, \bar{u}}^{\mathrm{D}}, \mathcal{H} \Psi_{L+2 b, \bar{u}}^{\mathrm{D}}\right\rangle \leq\left\langle\Psi_{L}, \mathcal{H} \Psi_{L}\right\rangle+\frac{C}{L b}\left\langle\Psi_{L}, \mathcal{N} \Psi_{L}\right\rangle
$$

for a universal constant $C>0$.

Proof. For an arbitrary $L$-periodic function $\psi \in L_{\text {loc }}^{2}(\mathbb{R})$, we find

$$
\int_{-\frac{L}{2}-b}^{\frac{L}{2}+b} d t|\psi(t)|^{2} q(t)^{2}=\int_{-\frac{L}{2}}^{\frac{L}{2}} d t|\psi(t)|^{2} .
$$

To prove (A.3), we combine (using the periodicity of $\psi$ ) the integral over $[-L / 2-b ;-L / 2]$ with the integral over $[L / 2-b ; L / 2]$ and the integral over $[-L / 2 ;-L / 2+b]$ with the integral over $[L / 2 ; L / 2+b]$ (using that $\cos ^{2} x+\cos ^{2}(x-\pi / 2)=1$ ). 
Applying (A.3) (separately on each variable), we obtain that $\left\|\left(\Psi_{L+2 b, u}^{\mathrm{D}}\right)^{(n)}\right\|=\left\|\Psi_{L}^{(n)}\right\|$, for all $n \in \mathbb{N}$. This implies that $\left\|\Psi_{L+2 b, u}^{\mathrm{D}}\right\|=\left\|\Psi_{L}\right\|=1$ and that $\left\langle\Psi_{L+2 b, u}^{\mathrm{D}}, \mathcal{N}^{j} \Psi_{L+2 b, u}^{\mathrm{D}}\right\rangle=\left\langle\Psi_{L}, \mathcal{N}^{j} \Psi_{L}\right\rangle$ for all $j \in \mathbb{N}$.

To compute the expectation of the kinetic energy in the state $\Psi_{L+2 b, u}^{\mathrm{D}}$, we observe that, for any $L$-periodic $\psi \in L_{\text {loc }}^{2}(\mathbb{R})$ with $\psi^{\prime} \in L_{\text {loc }}^{2}(\mathbb{R})$, we have (since $\psi^{\prime}$ is also $L$-periodic)

$$
\int_{-\frac{L}{2}-b}^{\frac{L}{2}+b} d t\left|(q \psi)^{\prime}(t)\right|^{2}=\int_{\frac{L}{2}}^{\frac{L}{2}} d t\left|\psi^{\prime}(t)\right|^{2}+\int_{-\frac{L}{2}-b}^{\frac{L}{2}+b} d t\left[|\psi(t)|^{2} q^{\prime}(t)^{2}+q(t) q^{\prime}(t) \frac{d}{d t}|\psi(t)|^{2}\right]
$$

where we used periodicity of $\psi^{\prime}$ and (A.3). Integrating by parts and using $q( \pm(L / 2+b))=q^{\prime}( \pm(L / 2-$ b)) $=0$, we get

$$
\begin{aligned}
\int_{-\frac{L}{2}-b}^{\frac{L}{2}+b} d t\left|(q \psi)^{\prime}(t)\right|^{2} & =\int_{-\frac{L}{2}}^{\frac{L}{2}} d t\left|\psi^{\prime}(t)\right|^{2}-\int_{-\frac{L}{2}-b}^{\frac{L}{2}+b} d t|\psi(t)|^{2} q(t) q^{\prime \prime}(t) \\
& \leq \int_{-\frac{L}{2}}^{\frac{L}{2}} d t\left|\psi^{\prime}(t)\right|^{2}+\frac{C}{b^{2}} \int_{\mathbb{R}} d t|\psi(t)|^{2} \chi_{L, b}(t)
\end{aligned}
$$

where $\chi_{L, b}(t)=\chi_{b}(t+L / 2)+\chi_{b}(t-L / 2)$ with $\chi_{r}(t)$ the characteristic function of $[-r, r]$ and we used $\left|q^{\prime \prime}(t)\right| \leq C b^{-2} \chi_{L, b}(t)$. Applying (A.4) (separately in every direction), we obtain

$$
\begin{aligned}
&\left\|\nabla_{x_{j}}\left(\Psi_{L+2 b, u}^{\mathrm{D}}\right)^{(n)}\right\|^{2} \\
& \leq\left\|\nabla_{x_{j}}\left(\Psi_{L+2 b, u}^{\mathrm{D}}\right)^{(n)}\right\|^{2} \\
&+\frac{C}{b^{2}} \int_{\mathbb{R}^{3}} d x_{j} \widetilde{\chi}_{L, b}\left(x_{j}-u\right) \int_{\Lambda_{L}^{n-1}} d x_{1} \ldots d x_{j-1} d x_{j+1} \ldots d x_{n}\left|\Psi_{L}^{(n)}\left(x_{1}, \ldots, x_{n}\right)\right|^{2}
\end{aligned}
$$

where we defined $\tilde{\chi}_{L, b}(x)=\sum_{k=1}^{3} \chi_{L, b}\left(x^{(k)}\right) \prod_{j \neq k}^{3} \chi_{\frac{L}{2}}\left(x^{(j)}\right)$.

To compute the potential energy of $\psi_{L}$, we have to consider the $L$-periodic extension $V_{L}(x)=$ $\sum_{m \in \mathbb{Z}^{3}} V(x+m L)$ of $V$. Since we assumed $V$ to be positive and supported in $B_{R}(0)$ and that $L>R$, we get $V(x) \leq V_{L}(x)$, which implies that, for any $i \neq j$,

$$
\begin{aligned}
& \left|\left(\Psi_{L+2 b, u}^{\mathrm{D}}\right)^{(n)}\left(x_{1}, \ldots, x_{n}\right)\right|^{2} V\left(x_{i}-x_{j}\right) \\
& \quad \leq\left|\Psi_{L}^{(n)}\left(x_{1}, \ldots, x_{n}\right) \sqrt{V_{L}\left(x_{i}-x_{j}\right)}\right|^{2} \prod_{k=1}^{n} Q_{L, b}\left(x_{k}-u\right)^{2} .
\end{aligned}
$$

Applying (A.3), we obtain

$$
\begin{aligned}
\int_{\left(\Lambda_{L+2 b}^{u}\right)^{n}} d x_{1} \ldots d x_{n} \mid & \left.\left(\Psi_{L+2 b, u}^{\mathrm{D}}\right)^{(n)}\left(x_{1}, \ldots, x_{n}\right)\right|^{2} V\left(x_{i}-x_{j}\right) \\
& \leq \int_{\Lambda_{L}^{n}} d x_{1} \ldots d x_{n}\left|\Psi_{L}^{(n)}\left(x_{1}, \ldots, x_{n}\right)\right|^{2} V_{L}\left(x_{i}-x_{j}\right) .
\end{aligned}
$$

From (A.5) and (A.6), we conclude (using the bosonic symmetry)

$$
\begin{aligned}
\left\langle\Psi_{L+2 b, u}^{\mathrm{D}},\right. & \left.\mathcal{H} \Psi_{L+2 b, u}^{\mathrm{D}}\right\rangle \\
& \leq\left\langle\Psi_{L}, \mathcal{H} \Psi_{L}\right\rangle+\frac{C}{b^{2}} \sum_{n \geq 0} n \int_{\mathbb{R}^{3}} d x_{1} \widetilde{\chi}_{L, b}\left(x_{1}-u\right) \int_{\Lambda_{L}^{n-1}} d x_{2} \ldots d x_{n}\left|\Psi_{L}^{(n)}\left(x_{1}, \ldots, x_{n}\right)\right|^{2} .
\end{aligned}
$$


Averaging over $u \in \Lambda_{L}$ we conclude (since $\left\|\widetilde{\chi}_{L, b}\right\|_{1} \leq C L^{2} b$ )

$$
\int_{\Lambda_{L}} d u\left\langle\Psi_{L+2 b, u}^{\mathrm{D}}, \mathcal{H} \Psi_{L+2 b, u}^{\mathrm{D}}\right\rangle \leq L^{3}\left\langle\Psi_{L}, \mathcal{H} \Psi_{L}\right\rangle+\frac{C L^{2}}{b}\left\langle\Psi_{L}, \mathcal{N} \Psi_{L}\right\rangle
$$

Hence, there exists $\bar{u} \in \Lambda_{L}$ so that (A.2) holds.

From now on, let us define $\Psi_{L+2 b}^{\mathrm{D}} \in \mathcal{F}\left(\Lambda_{L+2 b}\right)$, setting $\left(\Psi_{L+2 b}^{\mathrm{D}}\right)^{(n)}\left(x_{1}, \ldots, x_{n}\right)=\left(\Psi_{L+2 b, \bar{u}}^{\mathrm{D}}\right)^{(n)}\left(x_{1}-\right.$ $\left.\bar{u}, \ldots, x_{n}-\bar{u}\right)$, with $\Psi_{L+2 b, \bar{u}}^{\mathrm{D}}$ from Lemma A.1. Since $\Psi_{L+2 b}^{\mathrm{D}}$ satisfies Dirichlet boundary conditions, we can replicate it into several adjacent copies of $\Lambda_{L+2 b}$, separated by corridors of size $R$ (to avoid interactions between different boxes). This allows us to construct a sequence of trial states on boxes with increasing volume (but keeping the density fixed).

Let $t \in \mathbb{N}$ and $\tilde{L}=t(L+2 b+R)$. We think of the large box $\Lambda_{\tilde{L}}$ as the (almost) disjoint union of $t^{3}$ shifted copies of the small box $\Lambda_{L+2 b+R}$, centred at

$$
(-\tilde{L} / 2,-\tilde{L} / 2,-\tilde{L} / 2)+(L+2 b+R) \cdot\left(i_{1}-1 / 2, i_{2}-1 / 2, i_{3}-1 / 2\right)
$$

with $i_{1}, i_{2}, i_{3} \in\{1, \ldots, t\}$. Let $\left\{c_{i}\right\}_{i=1}^{t^{3}}$ denote an enumeration of the centres (A.7). We define $\Psi_{\tilde{L}}^{\mathrm{D}} \in$ $\mathcal{F}\left(\Lambda_{\tilde{L}}\right)$ by setting

$$
\left(\Psi_{\tilde{L}}^{\mathrm{D}}\right)^{(m)}\left(x_{1}, \ldots, x_{m}\right)=\frac{1}{\left\|\left(\Psi_{L+2 b}^{\mathrm{D}}\right)^{(n)}\right\|^{t^{3}-1}} \prod_{i=1}^{t^{3}}\left(\Psi_{L+2 b}^{\mathrm{D}}\right)^{(n)}\left(x_{(i-1) n+1}-c_{i}, \ldots, x_{i n}-c_{i}\right)
$$

if $m=n t^{3}$ for an $n \in \mathbb{N}$ and $\left(\Psi_{\tilde{L}}^{\mathrm{D}}\right)^{(m)}=0$ otherwise (here we set $\left(\Psi_{L+2 b}^{\mathrm{D}}\right)^{(n)}=0$ if one of its arguments lies outside $\Lambda_{L+2 b}$ ). More precisely, $\left(\Psi_{\tilde{L}}^{\mathrm{D}}\right)^{(m)}$ should be defined as the symmetrisation of (A.8) (but we can use (A.8) to compute the expectation of permutation symmetric observables).

Lemma A.2. Under the assumptions of Proposition 1.2, let $\Psi_{\tilde{L}}^{D}$ be defined as above. Then $\left\|\Psi_{\tilde{L}}^{D}\right\|=1$,

$$
\left\langle\Psi_{\tilde{L}}^{\mathrm{D}}, \mathcal{N}^{j} \Psi_{\tilde{L}}^{\mathrm{D}}\right\rangle=t^{3 j}\left\langle\Psi_{L+2 b}^{\mathrm{D}}, \mathcal{N}^{j} \Psi_{L+2 b}^{\mathrm{D}}\right\rangle
$$

for all $j \in \mathbb{N}$ and

$$
\left\langle\Psi_{\tilde{L}}^{\mathrm{D}}, \mathcal{H} \Psi_{\tilde{L}}^{\mathrm{D}}\right\rangle=t^{3}\left\langle\Psi_{L+2 b}^{\mathrm{D}}, \mathcal{H} \Psi_{L+2 b}^{\mathrm{D}}\right\rangle
$$

Proof. From the definition (A.8), we have $\left\|\left(\Psi_{\tilde{L}}^{\mathrm{D}}\right)^{\left(n t^{3}\right)}\right\|=\left\|\left(\Psi_{L+2 b}^{\mathrm{D}}\right)^{(n)}\right\|$ for all $n \in \mathbb{N}$. Since $\left(\Psi_{\tilde{L}}^{\mathrm{D}}\right)^{(m)}=$ 0 , if $m \neq n t^{3}$, we conclude that $\left\|\Psi_{\tilde{L}}^{\mathrm{D}}\right\|=\left\|\Psi_{L+2 b}^{\mathrm{D}}\right\|=1$ and also that, for $j \in \mathbb{N}$,

$$
\left\langle\Psi_{\tilde{L}}^{\mathrm{D}}, \mathcal{N}^{j} \Psi_{\tilde{L}}^{\mathrm{D}}\right\rangle=\sum_{n \geq 0}\left(t^{3} n\right)^{j}\left\|\left(\Psi_{\tilde{L}}^{\mathrm{D}}\right)^{\left(t^{3} n\right)}\right\|^{2}=t^{3 j} \sum_{n \geq 0} n^{j}\left\|\left(\Psi_{L+2 b}^{\mathrm{D}}\right)^{(n)}\right\|^{2}=t^{3 j}\left\langle\Psi_{L+2 b}^{\mathrm{D}}, \mathcal{N}^{j} \Psi_{L+2 b}^{\mathrm{D}}\right\rangle
$$

To prove (A.9), we observe, first of all, that for any $i=1, \ldots, n t^{3}$, when the operator $\nabla_{x_{i}}$ acts on $\left(\Psi_{\tilde{L}}^{\mathrm{D}}\right)^{\left(n t^{3}\right)}$, it only hits one of the factors $\left(\Psi_{L+2 b}^{\mathrm{D}}\right)^{(n)}$ on the right-hand side of (A.8). Similarly, for any $i, j \in\{1, \ldots, m\}$, the operator $V\left(x_{i}-x_{j}\right)$ has nonzero expectation in the state $\left(\Psi_{\tilde{L}}^{\mathrm{D}}\right)^{\left(n t^{3}\right)}$ only if $x_{i}, x_{j}$ are arguments of the same factor $\left(\Psi_{L+2 b}^{\mathrm{D}}\right)^{(n)}$ on the right-hand side of (A.8) (this observation is exactly the reason for introducing corridors of size $R$ between the small boxes, where the wave function vanishes). We conclude that $\left\langle\Psi_{\tilde{L}}^{\mathrm{D}}, \mathcal{H} \Psi_{\tilde{L}}^{\mathrm{D}}\right\rangle=t^{3}\left\langle\Psi_{L+2 b}^{\mathrm{D}}, \mathcal{H} \Psi_{L+2 b}^{\mathrm{D}}\right\rangle$, as claimed.

Finally, in Lemma A.4 we show how to obtain an upper bound for the ground state energy per particle in the canonical ensemble, starting from a trial state in the grand-canonical setting. Recall the notation 
$E(N, L)$ for the ground state energy of the Hamiltonian (1.1), describing $N$ particles in the box $\Lambda_{L}$, with Dirichlet boundary conditions. For $\rho>0$ with $\rho L^{3} \in \mathbb{N}$, we introduce the notation

$$
e_{L}(\rho)=\frac{E\left(\rho L^{3}, L\right)}{L^{3}}
$$

Comparing with the definition (1.2), we find $e(\rho)=\lim _{L \rightarrow \infty} e_{L}(\rho)$ (where the limit has to be taken along sequences of $L$, with $\rho L^{3} \in \mathbb{N}$ ). In the proof of Lemma A.4 we use the existence of the thermodynamic limit of the specific energy and its convexity (see [19, Thm. 3.5.8 and 3.5.11]), together with the following result on the Legendre transform of convex functions.

Lemma A.3. Let $D \subset \mathbb{R}$ be a closed interval and $f: D \rightarrow \mathbb{R}$ be convex and continuous (also at the boundary of $D$ ). We define the Legendre transform $f^{*}: \mathbb{R} \rightarrow \mathbb{R}$ off by

$$
f^{*}(y)=\sup _{x \in D}[x y-f(x)]
$$

Then $f^{*}$ is well-defined (because, by continuity, $x \rightarrow x y-f(x)$ is bounded on $D$, for all $y \in \mathbb{R}$ ) and, for all $x \in D$,

$$
f(x)=\sup _{y \in \mathbb{R}}\left[x y-f^{*}(y)\right] .
$$

Proof. By definition of $f^{*}$, we have $f^{*}(y) \geq x y-f(x)$ for all $x \in D, y \in \mathbb{R}$. This implies that $f(x) \geq x y-f^{*}(y)$ for all $x \in D, y \in \mathbb{R}$ and therefore that

$$
f(x) \geq \sup _{y \in \mathbb{R}}\left[x y-f^{*}(y)\right]
$$

for all $x \in D$. On the other hand, fix $x_{0} \in D$ and $t \leq f\left(x_{0}\right)$. Then, by convexity of $f$ (and by its continuity at the boundaries of $D$ ), we find a line through $\left(x_{0}, t\right)$ lying below the graph of $f$. In other words, there exists $y \in \mathbb{R}$ such that $f(x) \geq t+y\left(x-x_{0}\right)$ for all $x \in D$. Thus, $y x_{0}-t \geq y x-f(x)$ for all $x \in D$, which implies that

$$
y x_{0}-t \geq f^{*}(y)
$$

and therefore that $t \leq y x_{0}-f^{*}(y)$. In particular, $t \leq \sup _{y \in \mathbb{R}}\left[y x_{0}-f^{*}(y)\right]$. Since $t \leq f\left(x_{0}\right)$ was arbitrary, we conclude that $f\left(x_{0}\right) \leq \sup _{y \in \mathbb{R}}\left[y x_{0}-f^{*}(y)\right]$. With (A.12), we obtain that $f(x)=\sup _{y \in \mathbb{R}}\left[x y-f^{*}(y)\right]$ for all $x \in D$.

Lemma A.4. Under the assumptions of Proposition 1.2, fix $\rho>0$ and suppose that there exists a sequence $\Psi_{L}^{D} \in \mathcal{F}\left(\Lambda_{L}\right)$ (parametrised by $L$ with $\rho L^{3} \in \mathbb{N}$ ), satisfying Dirichlet boundary conditions, such that

$$
\left\langle\Psi_{L}^{D}, \mathcal{N} \Psi_{L}^{D}\right\rangle \geq \rho\left(1+c^{\prime} \rho\right) L^{3}, \quad\left\langle\Psi_{L}^{D}, \mathcal{N}^{2} \Psi_{L}^{D}\right\rangle \leq C^{\prime}\left(\rho L^{3}\right)^{2}
$$

for some constants $c^{\prime}, C^{\prime}>0$. Then we have

$$
e(\rho) \leq \lim _{L \rightarrow \infty} \frac{\left\langle\Psi_{L}^{D}, \mathcal{H} \Psi_{L}^{D}\right\rangle}{L^{3}} .
$$


Proof. Using positivity of $\mathcal{H}$, we have, for any $\mu \geq 0$ and $M>0$,

$$
\begin{aligned}
& \frac{\left\langle\Psi_{L}^{\mathrm{D}}, \mathcal{H} \Psi_{L}^{\mathrm{D}}\right\rangle}{L^{3}} \\
& \geq \frac{\mu}{L^{3}}\left\langle\Psi_{L}^{\mathrm{D}}, \mathcal{N} \Psi_{L}^{\mathrm{D}}\right\rangle+\frac{\left\langle\Psi_{L}^{\mathrm{D}},(\mathcal{H}-\mu \mathcal{N}) \chi\left(\mathcal{N} \leq M L^{3}\right) \Psi_{L}^{\mathrm{D}}\right\rangle}{L^{3}}-\frac{\mu}{L^{3}}\left\langle\Psi_{L}^{\mathrm{D}}, \mathcal{N} \chi\left(\mathcal{N}>M L^{3}\right) \Psi_{L}^{\mathrm{D}}\right\rangle \\
& \geq \frac{\mu}{L^{3}}\left\langle\Psi_{L}^{\mathrm{D}}, \mathcal{N} \Psi_{L}^{\mathrm{D}}\right\rangle+\sum_{m=0}^{M L^{3}}\left(e_{L}\left(\frac{m}{L^{3}}\right)-\mu \frac{m}{L^{3}}\right)\left\|\left(\Psi_{L}^{\mathrm{D}}\right)^{(m)}\right\|^{2}-\frac{\mu}{M L^{6}}\left\langle\Psi_{L}^{\mathrm{D}}, \mathcal{N}^{2} \Psi_{L}^{\mathrm{D}}\right\rangle,
\end{aligned}
$$

where we used the inequality $\chi\left(\mathcal{N}>M L^{3}\right) \leq \mathcal{N} /\left(M L^{3}\right)$. Hence, with (A.13) and fixing $M$ large enough (depending on $c^{\prime}, C^{\prime}$ ), we find

$$
\frac{\left\langle\Psi_{L}^{\mathrm{D}}, \mathcal{H} \Psi_{L}^{\mathrm{D}}\right\rangle}{L^{3}} \geq \mu \rho+\sum_{m=0}^{M L^{3}}\left(e_{L}\left(\frac{m}{L^{3}}\right)-\mu \frac{m}{L^{3}}\right)\left\|\left(\Psi_{L}^{\mathrm{D}}\right)^{(m)}\right\|^{2}
$$

Next, we claim that

$$
e_{L}(\rho) \geq\left(1+\frac{R}{L}\right)^{3} e\left(\rho\left(1+\frac{R}{L}\right)^{-3}\right)
$$

Indeed, starting from an arbitrary normalised trial state $\psi$ describing $N=\rho L^{3}$ particles in a box of side length $L$, with Dirichlet boundary conditions, we can construct, for any $r \in \mathbb{N}$, a trial state describing $N^{\prime}=N r^{3}=\rho L^{3} r^{3}$ particles in a box of side length $L^{\prime}=r(L+R)$, again with Dirichlet boundary conditions, by placing $r^{3}$ copies of the state $\psi$ in adjacent boxes and using that (thanks to the corridors of size $R$ between the boxes) particles in different boxes do not interact. This construction is very similar to the one presented around Lemma A.2 (the difference is that here we work in the canonical setting, which makes things slightly simpler). Since $N^{\prime}=\left[\rho /(1+R / L)^{3}\right] L^{\prime 3}$, optimising the choice of $\psi$, we obtain that $E\left(\left[\rho /(1+R / L)^{3}\right] L^{\prime 3}, L^{\prime}\right) \leq r^{3} E\left(\rho L^{3}, L\right)$ and therefore that

$$
e_{L^{\prime}}\left(\rho /(1+R / L)^{3}\right) \leq e_{L}(\rho) /(1+R / L)^{3} .
$$

Taking the limit $L^{\prime} \rightarrow \infty$ (along the sequence $L^{\prime}=r(L+R), r \in \mathbb{N}$ ), we obtain (A.16). Then (A.15) and (A.16) yield

$$
\frac{\left\langle\Psi_{L}^{\mathrm{D}}, \mathcal{H} \Psi_{L}^{\mathrm{D}}\right\rangle}{L^{3}} \geq \mu \rho-\left(1+\frac{R}{L}\right)^{3} e^{*}(\mu)
$$

where $e^{*}$ denotes the Legendre transform of $e: D \rightarrow \mathbb{R}$, defined on the domain $D=[0, M]$, as in (A.10) (here we use the convexity of the specific energy $e$ ). It follows that

$$
\lim _{L \rightarrow+\infty} \frac{\left\langle\Psi_{L}^{\mathrm{D}}, \mathcal{H} \Psi_{L}^{\mathrm{D}}\right\rangle}{L^{3}} \geq \mu \rho-e^{*}(\mu)
$$

for all $\mu \geq 0$. Thus,

$$
\lim _{L \rightarrow+\infty} \frac{\left\langle\Psi_{L}^{\mathrm{D}}, \mathcal{H} \Psi_{L}^{\mathrm{D}}\right\rangle}{L^{3}} \geq \sup _{\mu \geq 0}\left[\mu \rho-e^{*}(\mu)\right]=\sup _{\mu \in \mathbb{R}}\left[\mu \rho-e^{*}(\mu)\right]=e(\rho)
$$

where we used the fact that $e^{*}(0)=0$ (because $e(\rho) \geq 0$ for all $\rho \geq 0$ and $e(0)=0$ ) and $e^{*}(\mu) \geq$ $-e(0)=0$ for all $\mu \in \mathbb{R}$ in the second step and Lemma A.3 in the third step.

With Lemmas A.1, A.2 and A.4 we are ready to show Proposition 1.2. 
Proof of Proposition 1.2. Given a normalised $\Psi_{L} \in \mathcal{F}\left(\Lambda_{L}\right)$ satisfying periodic boundary conditions with

$$
\left\langle\Psi_{L}, \mathcal{N} \Psi_{L}\right\rangle \geq \rho\left(1+c^{\prime} \rho\right)(L+2 b+R)^{3}, \quad\left\langle\Psi_{L}, \mathcal{N}^{2} \Psi_{L}\right\rangle \leq C^{\prime} \rho^{2}(L+2 b+R)^{6}
$$

we find with Lemma A.1 a normalised $\Psi_{L+2 b}^{\mathrm{D}} \in \mathcal{F}\left(\Lambda_{L+2 b}\right)$ satisfying Dirichlet conditions such that

$$
\left\langle\Psi_{L+2 b}, \mathcal{N} \Psi_{L+2 b}\right\rangle \geq \rho\left(1+c^{\prime} \rho\right)(L+2 b+R)^{3}, \quad\left\langle\Psi_{L+2 b}, \mathcal{N}^{2} \Psi_{L+2 b}\right\rangle \leq C^{\prime} \rho^{2}(L+2 b+R)^{6}
$$

and

$$
\left\langle\Psi_{L+2 b}^{\mathrm{D}}, \mathcal{H} \Psi_{L+2 b}^{\mathrm{D}}\right\rangle \leq\left\langle\Psi_{L}, \mathcal{H} \Psi_{L}\right\rangle+\frac{C}{L b}\left\langle\Psi_{L}, \mathcal{N} \Psi_{L}\right\rangle
$$

With Lemma A.2, we obtain a sequence $\Psi_{\tilde{L}}^{\mathrm{D}} \in \mathcal{F}\left(\Lambda_{\tilde{L}}\right)$, with $\tilde{L}=t(L+2 b+R)$ for $t \in \mathbb{N}$, such that

$$
\left\langle\Psi_{\tilde{L}}^{\mathrm{D}}, \mathcal{N} \Psi_{\tilde{L}}^{\mathrm{D}}\right\rangle \geq \rho\left(1+c^{\prime} \rho\right) \tilde{L}^{3}, \quad\left\langle\Psi_{\tilde{L}}^{\mathrm{D}}, \mathcal{N}^{2} \Psi_{\tilde{L}}^{\mathrm{D}}\right\rangle \leq C^{\prime} \rho^{2} \tilde{L}^{6}
$$

and

$$
\left\langle\Psi_{\tilde{L}}^{\mathrm{D}}, \mathcal{H} \Psi_{\tilde{L}}^{\mathrm{D}}\right\rangle \leq t^{3}\left\langle\Psi_{L}, \mathcal{H} \Psi_{L}\right\rangle+\frac{C t^{3}}{L b}\left\langle\Psi_{L}, \mathcal{N} \Psi_{L}\right\rangle
$$

With Lemma A.4, we conclude that

$$
\begin{aligned}
e(\rho) & \leq \lim _{\tilde{L} \rightarrow \infty} \frac{\left\langle\Psi_{\tilde{L}}^{\mathrm{D}}, \mathcal{H} \Psi_{\tilde{L}}^{\mathrm{D}}\right\rangle}{\tilde{L}^{3}} \\
& \leq \frac{1}{(1+2 b / L+R / L)^{3}}\left[\frac{\left\langle\Psi_{L}, \mathcal{H} \Psi_{L}\right\rangle}{L^{3}}+\frac{C}{L^{4} b}\left\langle\Psi_{L}, \mathcal{N} \Psi_{L}\right\rangle\right] \\
& \leq \frac{\left\langle\Psi_{L}, \mathcal{H} \Psi_{L}\right\rangle}{L^{3}}+\frac{C}{L^{4} b}\left\langle\Psi_{L}, \mathcal{N} \Psi_{L}\right\rangle .
\end{aligned}
$$

Acknowledgement. We are grateful to C. Boccato and S. Fournais for valuable discussions. G.B. and S.C. gratefully acknowledge the support from the GNFM Gruppo Nazionale per la Fisica Matematica. B. S. gratefully acknowledges partial support from the NCCR SwissMAP, from the Swiss National Science Foundation through the Grant 'Dynamical and Energetic Properties of Bose-Einstein Condensates' and from the European Research Council through the ERC-AdG CLaQS.

Conflict of Interest: None.

\section{References}

[1] A. Aaen, The Ground State Energy of a Dilute Bose Gas in Dimension $N>3, \mathrm{PhD}$ thesis (Department of Mathematics, Aarhus University, 2014).

[2] C. Boccato, C. Brennecke, S. Cenatiempo and B. Schlein, 'Bogoliubov theory in the Gross-Pitaevskii limit', Acta Math. 222(2) (2019), 219-335.

[3] C. Boccato, C. Brennecke, S. Cenatiempo and B. Schlein, 'Optimal rate for Bose-Einstein condensation in the GrossPitaevskii regime', Commun. Math. Phys. 376 (2020), 1311-1395.

[4] N. N. Bogoliubov, 'On the theory of superfluidity', Izv. Akad. Nauk. USSR 11(77) (1947), J. Phys. (USSR) 11 (1947), 23.

[5] B. Brietzke, S. Fournais and J. P. Solovej, 'A simple 2nd order lower bound to the energy of dilute Bose gases', Commun. Math. Phys. 376 (2020), 323-351.

[6] B. Brietzke and J. P. Solovey, 'The second order correction to the ground state energy of the dilute Bose gas, Ann. Henri Poincaré 21 (2020), 571-626.

[7] E. Carlen, I. Jauslin and E. H. Lieb, 'Analysis of a simple equation for the ground state energy of the Bose gas', Pure Appl. Anal. 2 (2020), 659-684.

[8] F. J. Dyson, 'Ground-state energy of a hard-sphere gas', Phys. Rev. 106 (1957), 20-26. 
[9] L. Erdős, B. Schlein and H.-T. Yau, 'Ground-state energy of a low-density Bose gas: a second order upper bound', Phys. Rev. A 78 (2008), 053627.

[10] S. Fournais and J. P. Solovej, 'The energy of dilute Bose gases', Ann. Math. 192(3) (2020), 893-976.

[11] S. Fournais and J. P. Solovej, 'The energy of dilute Bose gases II: the general case, Preprint, 2021, arXiv: 2108.12022.

[12] M. Girardeau and R. Arnowitt, 'Theory of many-Boson systems: pair theory', Phys. Rev. 113 (1959), 755.

[13] A. Giuliani and R. Seiringer, 'The ground state energy of the weakly interacting Bose gas at high density', J. Stat. Phys. 135 (2009), 915.

[14] T. D. Lee, K. Huang and C. N. Yang, 'Eigenvalues and eigenfunctions of a Bose system of hard spheres and its lowtemperature properties', Phys. Rev. 106 (1957), 1135-1145.

[15] E. H. Lieb, 'Simplified approach to the ground-state energy of an imperfect Bose gas', Phys. Rev. 130 (1963), 2518-2528.

[16] E. H. Lieb and J. Yngvason, 'Ground state energy of the low density Bose gas', Phys. Rev. Lett. 80 (1998), $2504-2507$.

[17] M. Napiórkowski, R. Reuvers and J. P. Solovej, 'The Bogoliubov free energy functional I. Existence of minimizers and phase diagrams', Arch. Ration. Mech. Anal. 229(3) (2018), 1037-1090.

[18] D. W. Robinson, 'The thermodynamic pressure in quantum statistical mechanics', Lect. Notes Phys. 9 (1971), $42-74$.

[19] D. Ruelle, Statistical Mechanics: Rigorous Results, 3rd ed. (Imperial College Press and World Scientific London, UK, 1969).

[20] H.-T. Yau and J. Yin, 'The second order upper bound for the ground state energy of a Bose gas', J. Stat. Phys. 136(3) (2009), 453-503. 